\title{
Application of a Bidirectional DC-DC in an Electric Powertrain for Medium Duty Delivery Trucks
}

\author{
Ali Najmabadi \\ Department of Electrical \& Computer Engineering \\ McGill University \\ Montreal, Quebec
}

April 2016

A thesis submitted to McGill University in partial fulfillment of the requirements of the degree of Master of Engineering

(C)Ali Najmabadi 2016 


\section{ACKNOWLEDGEMENTS}

This thesis would have been impossible to write if it was not for the help and support of many of my family members, colleagues and friends. I would like to thank my parents, Farzaneh and Farhang Najmabadi for their everlasting support throughout my life, especially during the last two years while I was working on my Masters. I would like to thank my girlfriend, Martine Anderson, who not only has provided me with lots of moral support in the past two years, she has also actively helped with writing of the four publications that have come together in this thesis. A special thanks to my supervisor Professor Benoit Boulet, who accepted me as his graduate student under a very special circumstances, provided me with ample growth opportunities and allowed me to pursue a topic that I was truly passionate about. I would also like to thank my colleague and friend, Subhadeep Bhattacharya. Without his technical and moral guidance this work would have never been achieved. I would like to give a very special thanks to Kieran Humphries. He has been my co-author for all my papers. Not only he has contributed immensely to the technical and graphical content of our papers and this thesis, his patience and willingness to accommodate radical work hours and deadlines are the reasons why this work was achievable in such a short time span. Lastly, I would like to thank all my friends. There are so many of you and I cannot mention everyone's names, but you all have contributed to this work. Thank you! 


\begin{abstract}
The efficiency of electric powertrains is of great importance for the electrification of transport. Different powertrain topologies have various advantages and disadvantages. In this thesis, the efficiencies of two different powertrains are compared. Both designs use a 2 level inverter and a surface mounted Permanent Magnet Synchronous Motor (PMSM). In the first design, the inverter is directly powered by a high voltage battery and is operated using traditional Pulse-Width Modulation (PWM). In the second design, a bidirectional DC-DC converter is used to connect a lower voltage battery to the inverter and is operated using a variable DC link voltage strategy at different Modulation Indices (MI) and battery voltages. Both powertrains are designed to operate a class 4 delivery truck, which uses two separate motors to power the rear wheels independently through two single-speed gearboxes. The efficiency maps of both systems are examined in the context of different drive cycles for a delivery truck. Furthermore, standard and experimentally captured drive cycles are compared and the results demonstrate the conditions under which the new system is most effective.
\end{abstract}




\begin{abstract}
ABRÉGÉ
L'efficacité des systèmes de propulsion électrique est d'une importance primordiale pour électrifier le réseau des transports. Chaque topologie différente a ses avantages et ses désavantages. Cette thèse a pour but de comparer l'efficacité de deux de ces systèmes. Les deux utilisent un moteur synchrone à aimant permanent. Le premier est contrôlé par un système de modulation de largeur d'impulsion (MLI) qui est connecté directement à la batterie. Le deuxième est contrôlé aussi par un système de modulation de largeur d'impulsion mais est connecté à un lienDC variable qui opère à différents indices de modulation et voltages de batterie. Ces systèmes sont conçus pour être utilisés dans des camions de livraison qui sont propulsés par deux moteurs liés aux roues arrières. Chacune des roues arrières est contrôlée indépendamment par un moteur et une boîte de transmission à une vitesse. L'efficacité des deux systèmes a été déterminée en utilisant des cycles de conduite identiques à ceux d'un camion de livraison. De plus, des modèles de cycle standardisés et expérimentaux ont été comparés et les résultats démontrent les conditions sous lesquelles le deuxième système est efficace.
\end{abstract}




\section{TABLE OF CONTENTS}

ACKNOWLEDGEMENTS ..................... . . ii

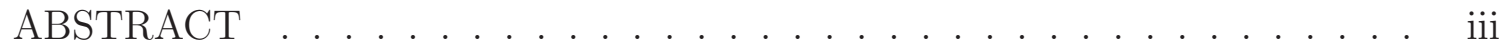

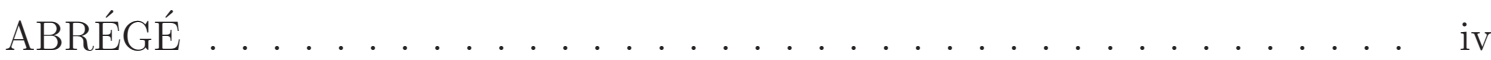

LIST OF TABLES . . . . . . . . . . . . . . . . . . vii

LIST OF FIGURES . . . . . . . . . . . . . . . . . . . . viii

1 Introduction . . . . . . . . . . . . . . . . . . 1

1.1 Environmental challenges and potential solutions . . . . . . . . . 1

1.2 Automotive Partnerships Canada Project . . . . . . . . . . . . . 1

1.3 Problem definition .................. . . 2

1.4 Associated Publications . . . . . . . . . . . . . . . . . 3

1.5 Thesis Outline . . . . . . . . . . . . . . . . . . 4

2 Background Information . . . . . . . . . . . . . . . 5

2.1 Park's Transformation .................. 5

2.2 Torque-Speed envelope of a motor . . . . . . . . . . . . . . 6

2.3 Mechanical vs. Electrical angular frequency of a motor . . . . . . 8

2.4 Control of a surface mounted PMSM . . . . . . . . . . . . . . . . 9

2.5 Application of power electronics in motor drives . . . . . . . . . . 12

2.6 Total harmonic distortion . . . . . . . . . . . . . . . . . . . . 12

2.7 Pulse-Width Modulation . . . . . . . . . . . . . . . . . 13

2.8 Modulation Index . . . . . . . . . . . . . . . . . . . . 17

2.9 Three-phase PWM waveforms and harmonic spectrum . . . . . . . 19

2.10 Overall Control of the system with PWM . . . . . . . . . . . . . . 19

2.11 Half Bridge DC-DC converter . . . . . . . . . . . . . . 20

2.12 Quantification of Drive Cycles .............. . 24 
3 Literature Review . . . . . . . . . . . . . . . . . . . . . . 26

3.1 Linkage of Energy Storage and Energy Conversion Systems (ESECS) 26

3.2 Increase of the efficiency in certain regions of operation . . . . . 28

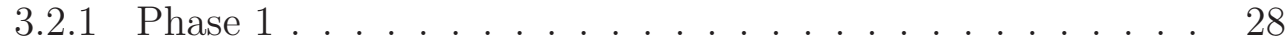

3.2 .2 Phase $2 \ldots \ldots \ldots \ldots \ldots \ldots$

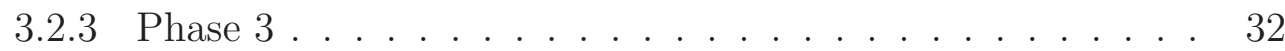

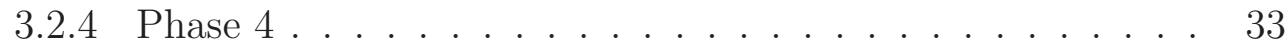

4 Methodology for Simulation . . . . . . . . . . . . . . . . . . . 34

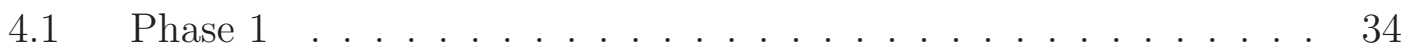

$4.1 .1 \quad$ Baseline, $\mathrm{S} 1 \ldots \ldots \ldots \ldots$

4.1 .2 New system, S2 . . . . . . . . . . . . . . . . . . 44

4.1.2.1 Overall System design . . . . . . . . . . . . . . 44

4.1.2.2 DC-DC converter design . . . . . . . . . . . 45

4.1.2.3 Control Strategy . . . . . . . . . . . . . . . 46

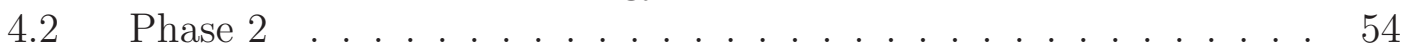

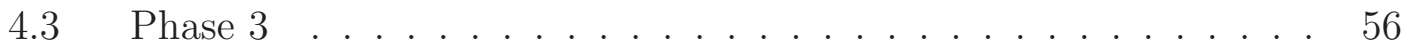

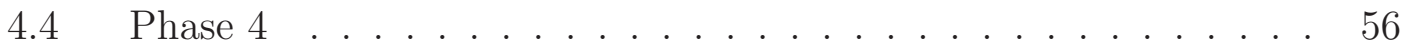

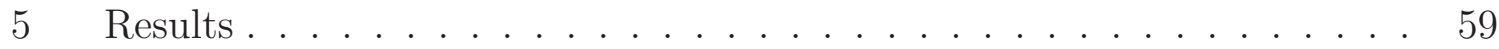

$5.1 \quad$ Phase $1 \ldots \ldots \ldots \ldots \ldots$

$5.2 \quad$ Phase $2 \ldots \ldots \ldots \ldots \ldots \ldots \ldots \ldots$

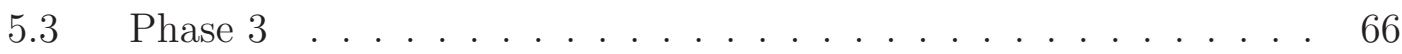

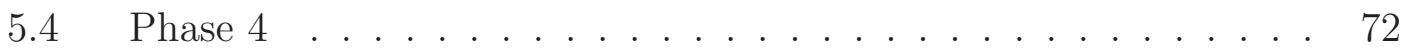

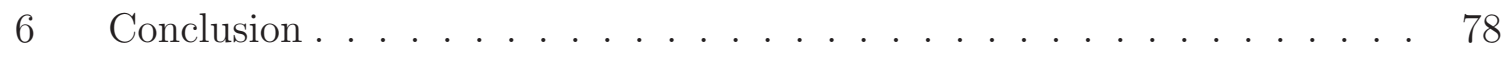

References. . . . . . . . . . . . . . . . . . . . . . . 82 
$4-1$ Simulation Parameters. . . . . . . . . . . . . . . . . . 43

5-1 Drive cycle metrics and vehicle simulation results for standard and recorded drive cycles for Phase $2 \ldots \ldots 66$

$5-2$ Vehicle simulation results for Phase $3 \ldots \ldots \ldots 71$

5-3 Drive cycle metrics with Phases 2 and $3 \ldots \ldots \ldots$

5-4 Vehicle simulation results for Phase $4 \ldots \ldots \ldots$

5-5 Drive cycle metrics and results for Phases 2,3 and $4 \ldots \ldots 77$ 
1-1 S1, traditional PWM battery-powered inverter . . . . . . . . . . 2

1-2 S2, variable DC bus voltage powered inverter . . . . . . . . . . . 3

2-1 PMSM Phase Voltage for different operation points . . . . . . . . 7

2-2 Torque-Speed Envelope . . . . . . . . . . . . . . . . . 7

2-3 Decoupling Stage . . . . . . . . . . . . . . . . . . 9

2-4 Main Control Stage with Flux Weakening . . . . . . . . . . . . . 10

2-5 Overall Control . . . . . . . . . . . . . . . . . . 10

2-6 Overall System . . . . . . . . . . . . . . . . . . 11

2-7 S1, traditional PWM battery-powered inverter . . . . . . . . . . 12

2-8 S1 with modified capacitor . . . . . . . . . . . . . . . 14

2-9 Single Phase PWM . . . . . . . . . . . . . . . . . . 15

2-10 Three Phase PWM . . . . . . . . . . . . . . . . . 16

2-11 Normalized Line to Line Voltage vs. MI . . . . . . . . . . . . . . . . . 19

2-12 PWM harmonic spectrum . . . . . . . . . . . . . . 20

2-13 Overall System Control with PWM . . . . . . . . . . . . . . . . 21

2-14 DC-DC Boost Converter . . . . . . . . . . . . . . . 22

2-15 Sample operation for a boost converter . . . . . . . . . . . . . . 23

3-1 Control Strategy for traditional PWM controlled motor . . . . . . . 29

3-2 Variable DC Bus Voltage Control with Target MI of '1' . . . . . . . . 30 
3-3 Variable DC Bus Voltage Control with Target MI of '1.5' . . . . . . . 32

4-1 Overall System Implementation in Simulink . . . . . . . . . . . . . . 36

4-2 Overall Control with Flux Weakening in Simulink . . . . . . . . . . . 37

4-3 Inverter Model in PSIM . . . . . . . . . . . . . . . . . . . 38

4-4 Motor angle, speed and torque for operation point of $100 \mathrm{NM}$ and $1000 \mathrm{RPM} \ldots \ldots . \ldots . \ldots . \ldots . \ldots 39$

4-5 Motor input and output power and the inverter's losses . . . . . . . . 40

$4-6$ Motor Currents . . . . . . . . . . . . . . . . . . . . . . 41

$4-7$ Motor Currents in steady-state . . . . . . . . . . . . . . . 42

$4-8$ Vehicle Model in AVL Cruise . . . . . . . . . . . . . . . . . . 44

4-9 Overall control strategy for a variable DC bus voltage control . . . . . 47

4-10 Decoupled System . . . . . . . . . . . . . . . . . . . . . . . . . 48

4-11 Zones of operation for S2 . . . . . . . . . . . . . . . . . . . . . . . 49

4-12 Control of the DC-DC converter in Matlab . . . . . . . . . . . . . 51

4-13 Implementation of the DC-DC converter in PSIM . . . . . . . . . . . 51

4-14 Sample operation of the DC-DC converter . . . . . . . . . . . 52

$4-15$ DC-DC converter Losses . . . . . . . . . . . . . . . . . . 53

$4-16$ Orange County Cycle . . . . . . . . . . . . . . . . . 5 54

4-17 Speed traces of standard drive cycles: NYCC, OCC, HTUF4-S, UDDS, and UDDSHD. . . . . . . . . . . . . 57

4-18 Speed traces of recorded daily drive cycles for delivery trucks in Anjou, Lachine, and Laval. . . . . . . . . . . . . . . . . . 58

$5-1 \quad$ Efficiency maps for $\mathrm{S} 1 \quad \ldots \ldots$. . . . . . . . . . . . . 62

$5-2$ Efficiency maps for $\mathrm{S} 2 \ldots \ldots \ldots \ldots$. . . . . . . . . . . . . 62 
5-3 Efficiency difference between $\mathrm{S} 1$ and $\mathrm{S} 2 \ldots \ldots$. . . . . . . . . 63

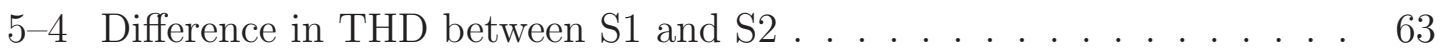

$5-5$ Operating point maps for OCC . . . . . . . . . . . . 63

5-6 Operating point maps for all cycles, standard cycles on the left and road tests on the right. . . . . . . . . . . . . . . 65

$5-7$ PED map for various MIs . . . . . . . . . . . . . . . . . 68

5-8 PTD map for various MIs . . . . . . . . . . . . . . . . . . . . 68

5-9 Energy consumption of S2 for various MI values during standard drive cycles ............................. 69

5-10 Energy consumption of S2 for various MI values during captured test cycles .......................... 70

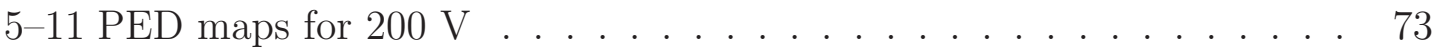

5-12 PED maps for $300 \mathrm{~V} \ldots \ldots . \ldots \ldots$. . . . . . . . . . . . . . . . . .

5-13 Energy consumption of S2 for various MIs and battery voltages during standard drive cycles . . . . . . . . . . . . . . . . . . . 74

5-14 Energy consumption of S2 for various MIs and battery voltages during captured test cycles . . . . . . . . . . . . . . . 75 


\section{Chapter 1 \\ Introduction}

\subsection{Environmental challenges and potential solutions}

Increase in $\mathrm{CO}_{2}$ levels has caused major global climate change in the recent years. This increase can be linked to the use of fossil fuels for heating, industrial applications and transportation. Within the transportation industry, there has been a movement to reduce the $\mathrm{CO}_{2}$ emissions with the introduction of alternative fuel vehicles. It has been shown that Hybrid Electric Vehicles (HEVs) and Battery

Electric Vehicles (BEVs) can help decrease $\mathrm{CO}_{2}$ emissions from transportation. Of course, well to wheel analysis is needed in order to include the emissions caused during the production and transportation of gas and electricity that are needed to operate these vehicles [1]. In cases that BEVs and HEVs can be beneficial, the efficiency of the vehicle itself becomes of utmost importance to minimize the emissions.

\subsection{Automotive Partnerships Canada Project}

The work presented in this thesis was completed at McGill University and is part of the larger Automotive Partnerships Canada (APC) project. One of APC's main goals is to increase the efficiency of electric drivetrains. APC is a collaboration between the Canadian government and many universities in Canada. The funding

for the project comes from the Natural Sciences and Engineering Research Council (NSERC) as well as industry partners: TM4, Infolytica, Purolator and Linamar. 


\subsection{Problem definition}

This thesis studies two different drive topologies for electric vehicles, S1 and S2. S1 includes a traditional battery powered 2 level inverter, which is controlled using Pulse-Width Modulation (PWM). This system will be used as the baseline for comparison purposes; its topology is shown in Figure 1-1. The second system, S2, uses a lower voltage battery and a half bridge boost converter to change the DC bus voltage of the 2 level inverter, as shown in Figure 1-2. The control strategy and the battery voltage of S2 are also subject to change and different combinations of these parameters will be studied. Both systems are used to operate a surface mounted Permanent Magnet Synchronous Motor (PMSM).

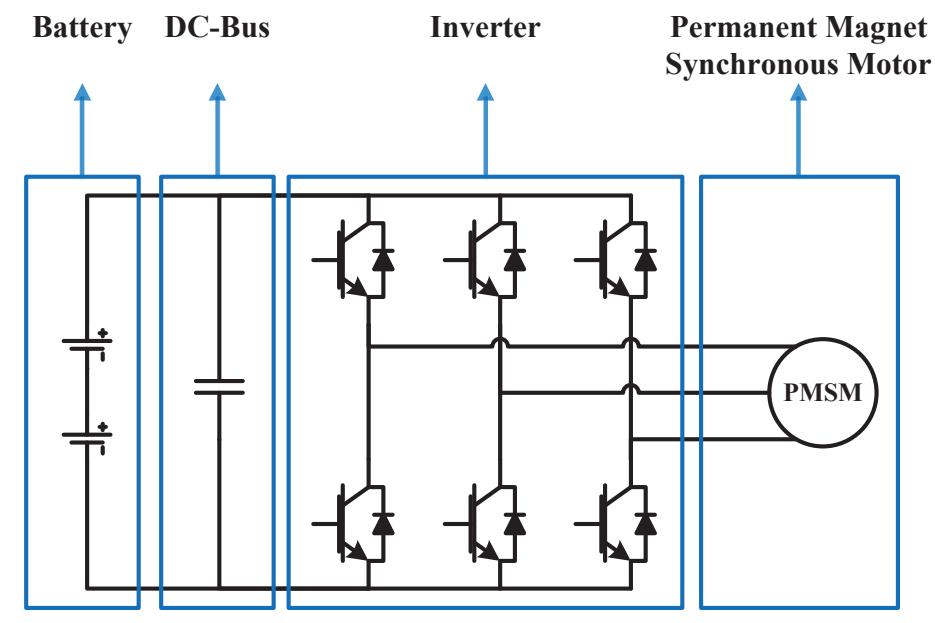

Figure 1-1: S1, traditional PWM battery-powered inverter

These systems are simulated to obtain powertrain efficiency data that is used to model the operation of a class 4 delivery truck over standard drive cycles, as well as real routes captured using a Purolator delivery truck in the greater Montreal area. 


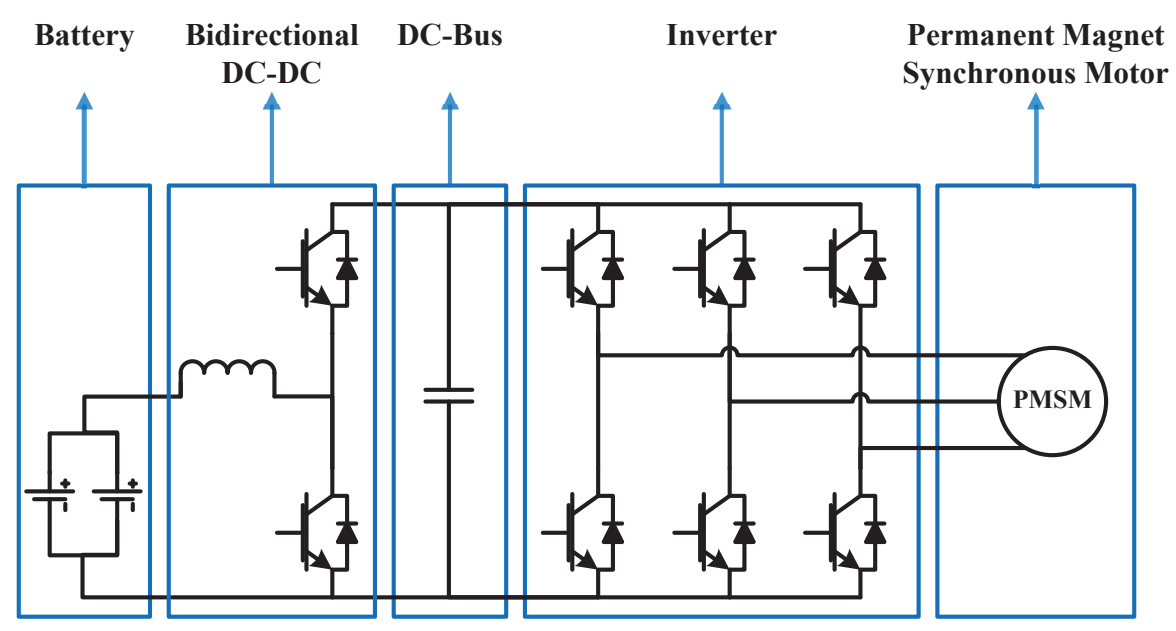

Figure 1-2: S2, variable DC bus voltage powered inverter

\subsection{Associated Publications}

The work presented in this thesis has be published in the following three papers:

- A. Najmabadi, K. Humphries, and B. Boulet, Application of a bidirectional dcdc in an electric powertrain for medium duty delivery trucks, in International Electric Machines and Drives Conference (IEMDC), 2015 IEEE, pp. 866871, IEEE. [2]

- A. Najmabadi, K. Humphries, and B. Boulet, Implementation of a bidirectional dc-dc in electric powertrains for drive cycles used by medium duty delivery trucks, in Energy Conversion Congress and Exposition (ECCE), 2015 IEEE, pp. 1338-1345, IEEE. [3]

- A. Najmabadi, K. Humphries, and B. Boulet, Effects of target modulation index on variable dc voltage control for electric powertrains in medium duty delivery trucks, in Vehicle Power and Propulsion Conference (VPPC), 2015 IEEE, IEEE. [4] 
The last part of this thesis has been submitted as a digest to the 2016 IEEE Transportation Electrification Conference and Expo (ITEC). The title of this digest is "Optimization of a Variable DC Bus Voltage Control Powertrain for Medium Duty Delivery Trucks for Various Drive Cycles". At the time of this thesis submission, the paper has yet to be accepted for the conference.

\subsection{Thesis Outline}

The next five chapters of this thesis are organized in the following manner. Chapter 2 will cover some important background information about electric motor drives and power electronics which will be crucial for understanding the overall work. Afterwards the main literature review will be presented in Chapter 3. Subsequently, the methodology used for different parts of the project will be explored in Chapter 4 . Finally, Chapter 5 will demonstrate the results and analysis and Chapter 6 will cover the conclusion of the overall project. 


\section{Chapter 2 \\ Background Information}

\subsection{Park's Transformation}

Park's Transformation is used to convert a three phase AC system in a rotating frame to a two-quantity DC system in a stationary frame. From now on the three phase AC system will be referred to as "abc frame" and the Park's transformation will be expressed in the direct and the quadrature axis known as the "dq frame" [5-7]. This transformation will be used extensively for converting the three phase $\mathrm{AC}$ voltages and currents to their dq frame counterparts. The following matrix operation transforms the system from abc to dq:

$$
\left[\begin{array}{lll}
V_{d} & V_{q} & V_{0}
\end{array}\right]=\left[\begin{array}{lll}
V_{a} & V_{b} & V_{c}
\end{array}\right] \frac{2}{3}\left[\begin{array}{ccc}
\cos (\theta) & -\sin (\theta) & \frac{1}{2} \\
\cos \left(\theta-\frac{2 \pi}{3}\right) & -\sin \left(\theta-\frac{2 \pi}{3}\right) & \frac{1}{2} \\
\cos \left(\theta+\frac{2 \pi}{3}\right) & -\sin \left(\theta+\frac{2 \pi}{3}\right) & \frac{1}{2}
\end{array}\right]
$$

Where, $V_{a}, V_{b} \& V_{c}$ are the time variant three phase voltages, $V_{q}, V_{d} \& V_{0}$ are the dq version of the three phase voltages and $\theta$ is the electrical angle. $V_{a}, V_{b} \& V_{c}$ are sinusoidal and balanced. It is important to note that $V_{0}$ is always zero and is omitted most of the times in the control loops. In order to transform the dq frame back to the abc frame, the following transformation is used. 


$$
\left[\begin{array}{lll}
V_{a} & V_{b} & V_{c}
\end{array}\right]=\left[\begin{array}{lll}
V_{d} & V_{q} & V_{0}
\end{array}\right]\left[\begin{array}{ccc}
\cos (\theta) & \cos \left(\theta-\frac{2 \pi}{3}\right) & \cos \left(\theta+\frac{2 \pi}{3}\right) \\
-\sin (\theta) & -\sin \left(\theta-\frac{2 \pi}{3}\right) & -\sin \left(\theta+\frac{2 \pi}{3}\right) \\
1 & 1 & 1
\end{array}\right]
$$

Furthermore, the following relationship can be written:

$$
\sqrt{V_{d}^{2}+V_{q}^{2}}=\hat{V}_{a}=\hat{V}_{b}=\hat{V}_{c}
$$

In this equation, $\hat{V}_{a}, \hat{V}_{b} \& \hat{V}_{c}$ are respectively the peak of voltages in the abc frame.

\subsection{Torque-Speed envelope of a motor}

Every motor can produce a certain amount of torque at a given speed. The first part of a torque-speed envelope of a motor is called the constant torque region (Figure 2-1). In case of the surface mounted PMSM, the phase voltage of the motor is ramped up with frequency which causes the motor speed to increase. Throughout this operation the torque is kept constant until the base speed of the motor is reached. From this point forward, the peak power of the motor is available, the motor will operate in the constant power region and the phase voltage of the motor will be kept constant. In order to increase the motor speed beyond its base speed, the torque needs to be lowered in order to keep the power constant. This is done by Flux weakening [1]. Figure 2-2 shows a generic torque-speed envelope. 


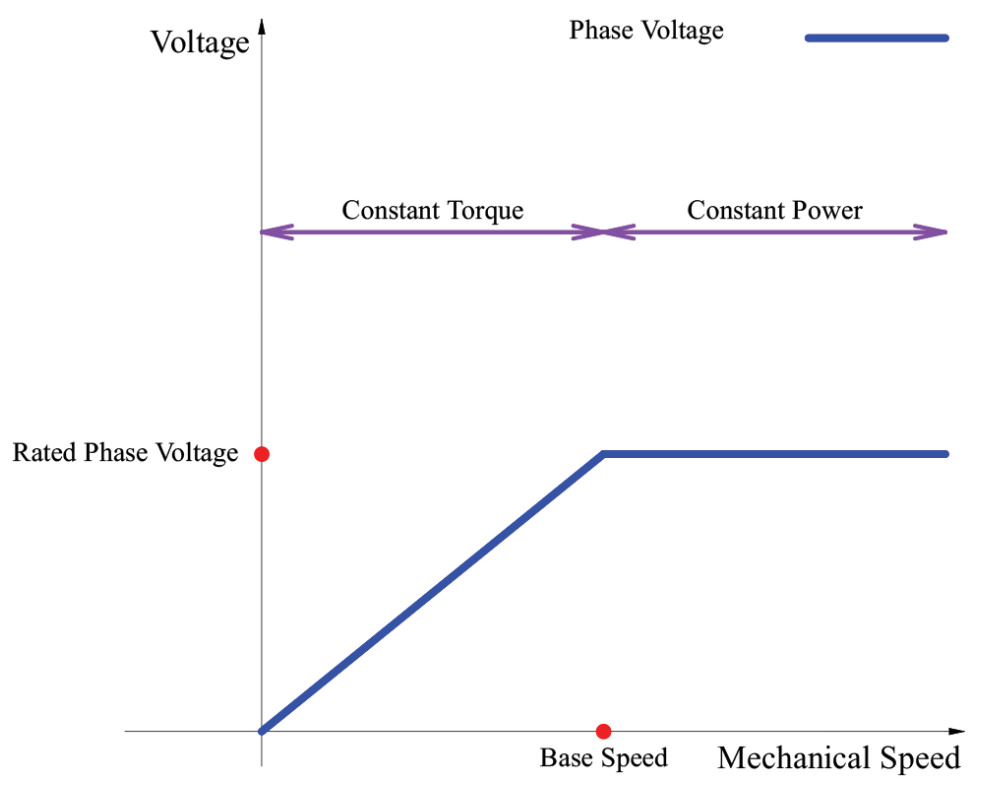

Figure 2-1: PMSM Phase Voltage for different operation points

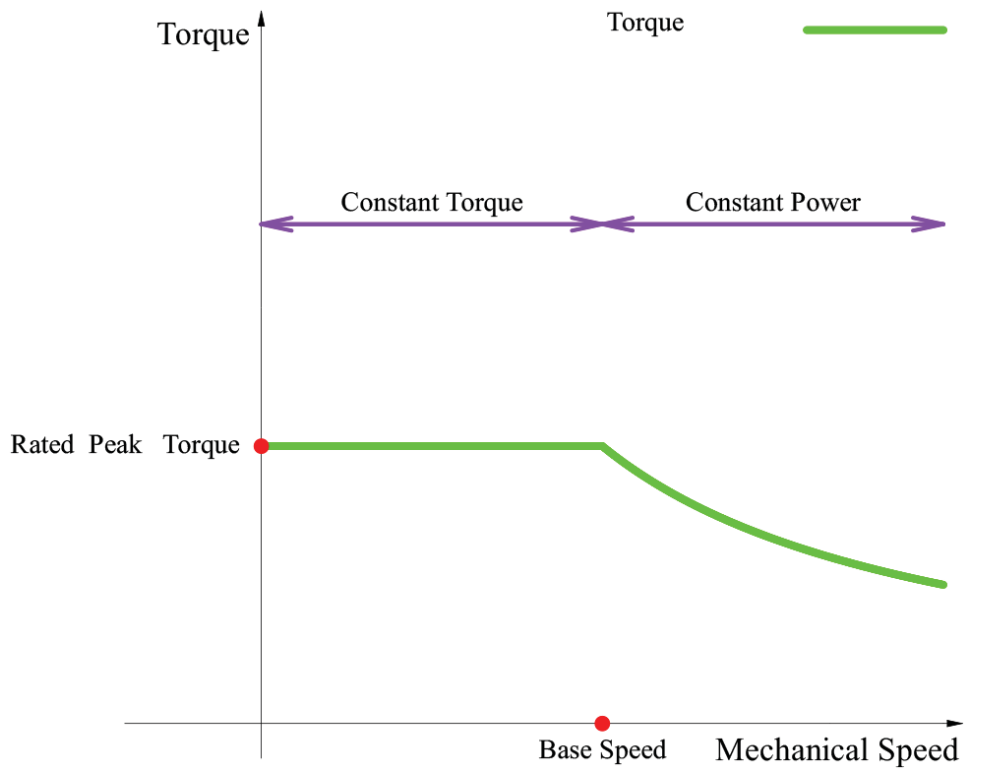

Figure 2-2: Torque-Speed Envelope 


\subsection{Mechanical vs. Electrical angular frequency of a motor}

The mechanical angular frequency $\left(\omega_{m}\right)$ of a motor can be obtained from its RPM by using the following formula [1]:

$$
\omega_{m}=R P M \times \frac{2 \pi}{60}
$$

Another way to obtain the mechanical angular frequency of a motor is by taking the derivative of the motor mechanical angle $\left(\theta_{m}\right)$ [1].The following equation describes this process:

$$
\omega_{m}=\frac{d \theta_{m}}{d t}
$$

The electrical angle $\left(\theta_{e}\right)$ and the electrical angular frequency $\left(\omega_{e}\right)$ that the motor currents and voltages operate at are different from the mechanical angle and mechanical angular frequency of the motor. The two types of angles and angular frequencies are related to each other by the number of the poles or number of pole pairs (PP) that the motor has. The following equations describe these quantities [1].

$$
\begin{gathered}
P P=\frac{\text { Numberof Poles }}{2} \\
\theta_{e}=P P \times \theta_{m} \\
\omega_{e}=P P \times \omega_{m}
\end{gathered}
$$




\subsection{Control of a surface mounted PMSM}

Load torque and reference speed $\left(\omega_{\text {ref }}\right)$ are the required set points for operation of a PMSM at a specific point in the torque-speed envelope. In the control diagrams, $\omega_{\text {ref }}$ and the load torque are shown in red boxes to demonstrate the fact that they are the desired set points. There are many different stages in the control of a PMSM [8]. The control will be described starting at the decoupling stage that uses the present value of the motor currents $\left(I_{d}, I_{q}\right)$, electrical angular frequency $\left(\omega_{e}\right)$, motor inductance $(L)$, the reference currents $\left(I_{d r e f}, I_{\text {qref }}\right)$ and two proportional and integral controllers $(P I)$ to produce the desired voltages $\left(V_{d}, V_{q}\right)$ that should be applied to the motor. Figure 2-3 represents the decoupling stage.

The next part is the main control stage with flux weakening. This stage uses the maximum possible phase voltage $\left(V_{s m}\right)$, maximum possible phase current $\left(I_{s m}\right)$, the desired phase voltages and the reference speed $\left(\omega_{r e f}\right)$ to produce the reference currents (Figure 2-4).

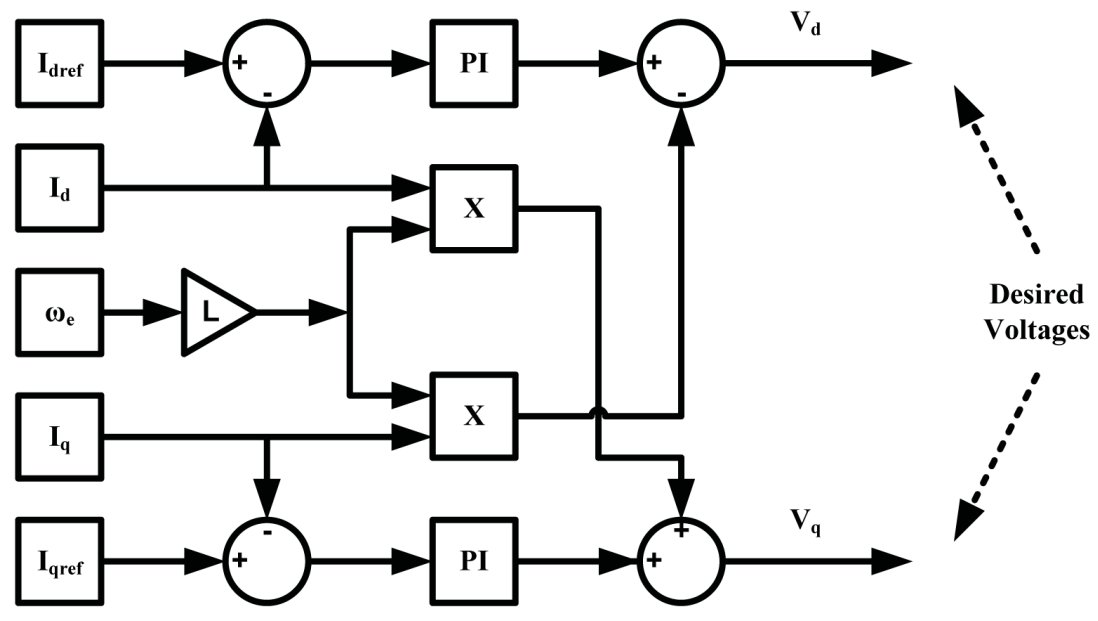

Figure 2-3: Decoupling Stage 


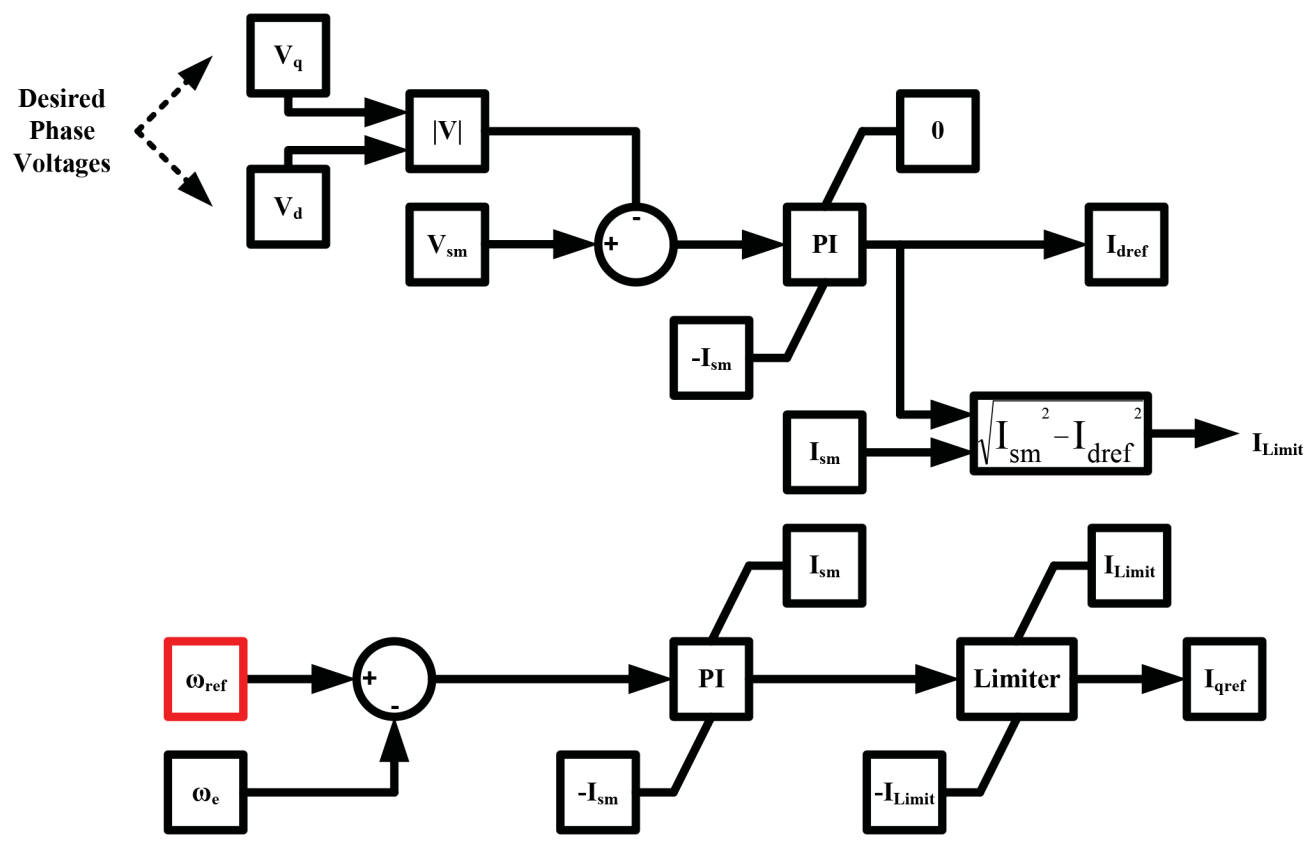

Figure 2-4: Main Control Stage with Flux Weakening

The overall control is demonstrated in Figure 2-5.

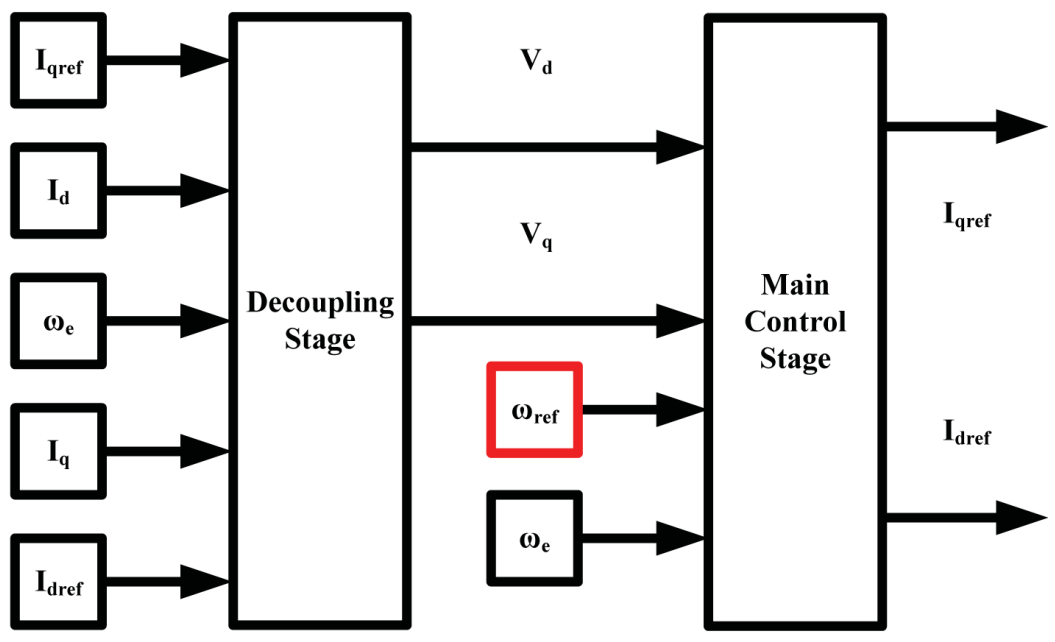

Figure 2-5: Overall Control

Finally, the overall system is shown in Figure 2-6: 


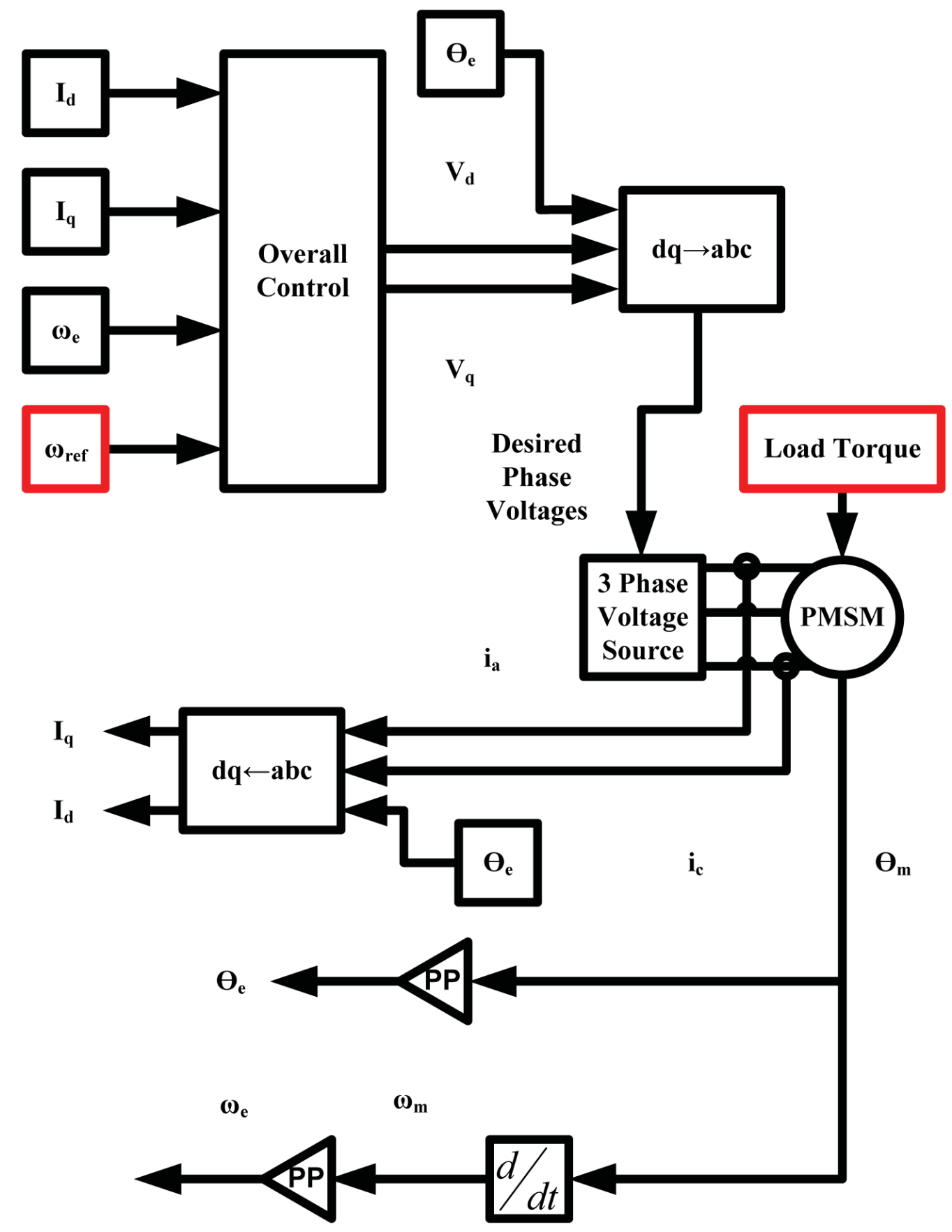

Figure 2-6: Overall System 


\subsection{Application of power electronics in motor drives}

When access to a three phase AC voltage source is not available, power electronics circuitry can be used in order to produce the desired waveforms from a DC source such as a battery. The most common topology uses a battery, a DC-link capacitor and a 2 level inverter [1]. The schematics of this topology are shown in Figure 2-7.

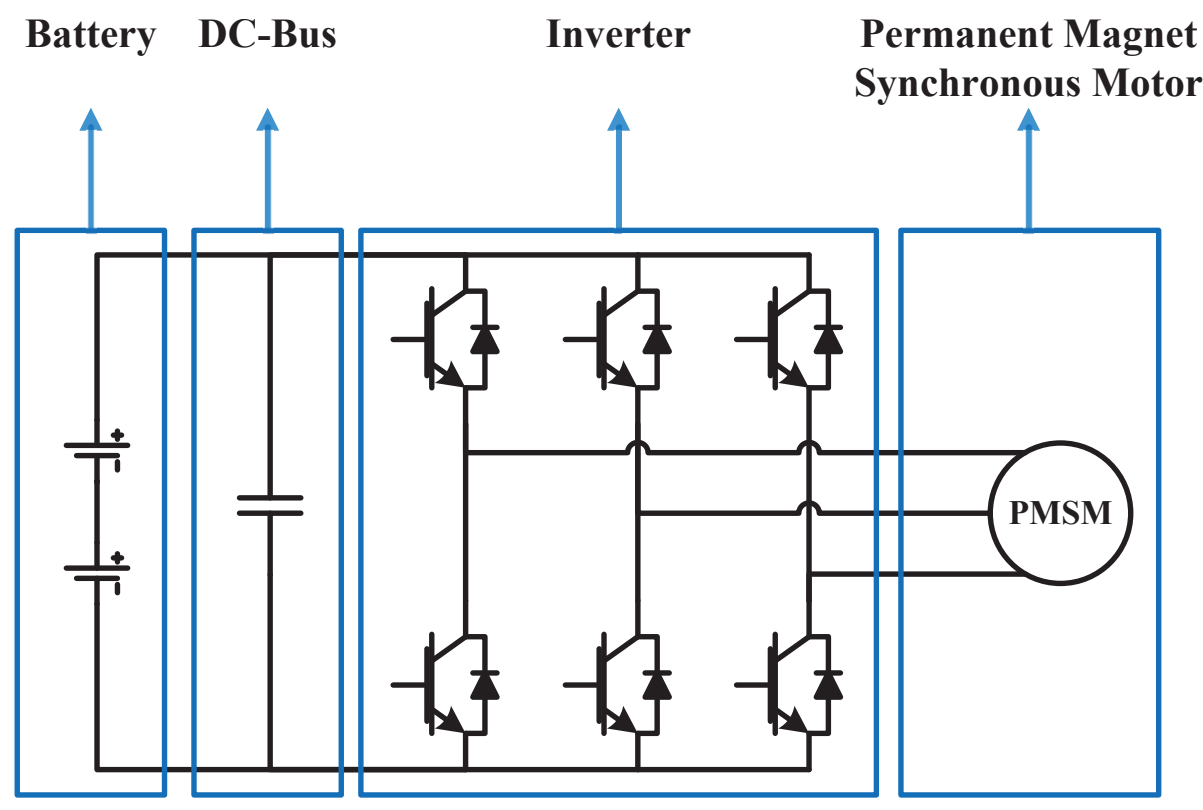

Figure 2-7: S1, traditional PWM battery-powered inverter

\subsection{Total harmonic distortion}

When using power electronics, the desired output waveforms are synthesized by pieces of the input waveforms. Therefore, in our example when we create an $\mathrm{AC}$ waveform from a DC voltage, the 3 phase line currents and voltages will not be pure sinusoids and they will contain some distortion. Fourier analysis can be used to isolate the desired fundamental waveform from the unwanted harmonics. 
The amount of distortion is quantified through an index named the Total Harmonic Distortion (THD) $[9,10]$. The following formulas show the derivation of the THD.

$$
\begin{gathered}
I_{s}^{2}=I_{s 1}^{2}+I_{s 2}^{2}+I_{s 3}^{2}+\ldots+I_{s n}^{2} \\
I_{d i s}=\sqrt{I_{s}^{2}-I_{s 1}^{2}} \\
T H D=100 \times \frac{I_{d i s}}{I_{s 1}}=100 \times \frac{\sqrt{I_{s}^{2}-I_{s 1}^{2}}}{I_{s 1}}
\end{gathered}
$$

Where $I_{s}$ is the line current, $I_{d i s}$ is the distortion on the line current and $I_{s 1}, I_{s 2} \ldots I_{s n}$ are the current harmonics. These harmonics are not desired in the system since they either contribute to the copper losses or the current ripples which result in torque ripples in an electric motor. Therefore, a lower THD is desirable.

\subsection{Pulse-Width Modulation}

There are different switching strategies that can be used to control an inverter and produce the desired phase voltages. Pulse-Width Modulation (PWM) is one of the most well-known strategies. In this control strategy, a scaled version of desired AC waveforms is compared to a triangular carrier. Whenever the control signal has a higher value than the triangular waveform, a transistor will turn on and the DC bus voltage will be available on the line, and whenever the control signal has a lower value than the triangular wave, the transistor will be turned off and the voltage on the line will go to zero. Therefore an on and off pattern will appear [9]. To understand

the concept of PWM, let us refer to Figure 2-8 which is the modified version of the 
classic 2 level inverter. In this system, the DC link capacitor is split in two to create a measuring point and we will consider the operation of one leg of the inverter and then we will expand this concept to all three legs.

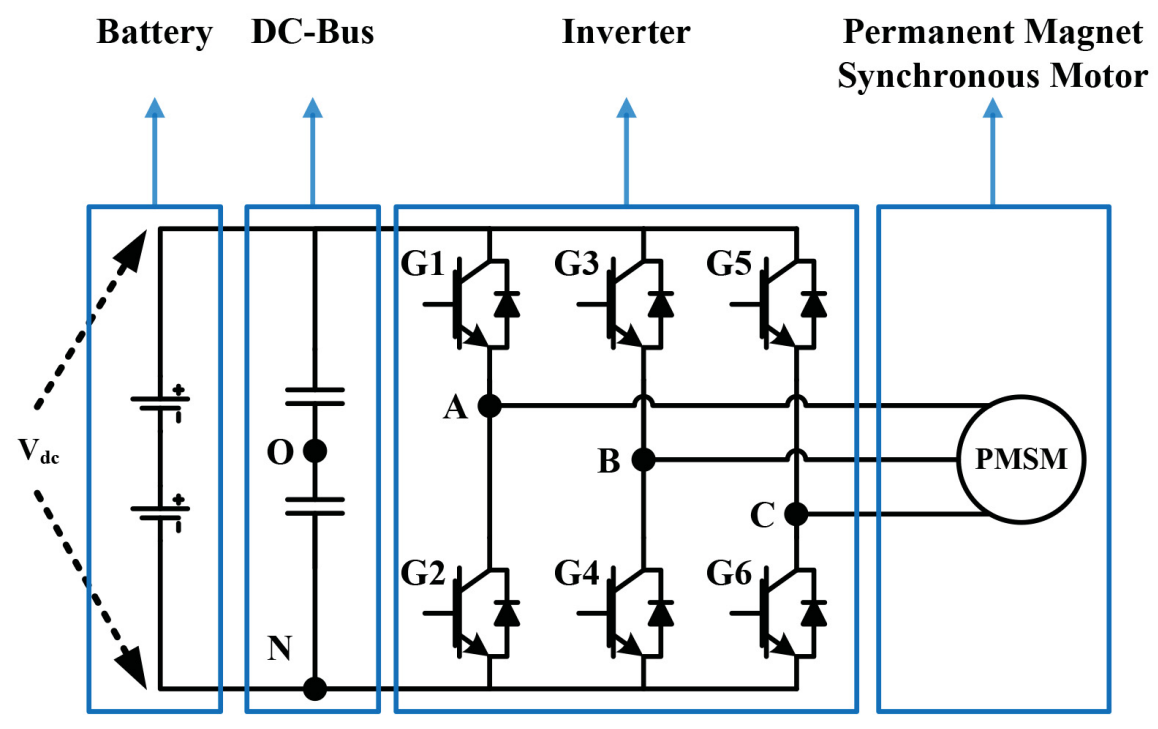

Figure 2-8: S1 with modified capacitor

In this case, we only have access to half of the DC bus voltage. For a DC bus of 400 Volts, the triangular wave and the control signal, the control signal of the switches, and the $V_{A O}$ are shown in Figure 2-9. The same idea can be expanded to the other phases which are $V_{B O}$ and $V_{C O}$. Furthermore, now that we know the single phase voltages, we can calculate the line to line voltage between phases A and $\mathrm{B}\left(V_{A B}\right)$ since:

$$
V_{A B}=V_{A O}-V_{B O}
$$

Figure 2-10 represents the behavior of the three phase system. 

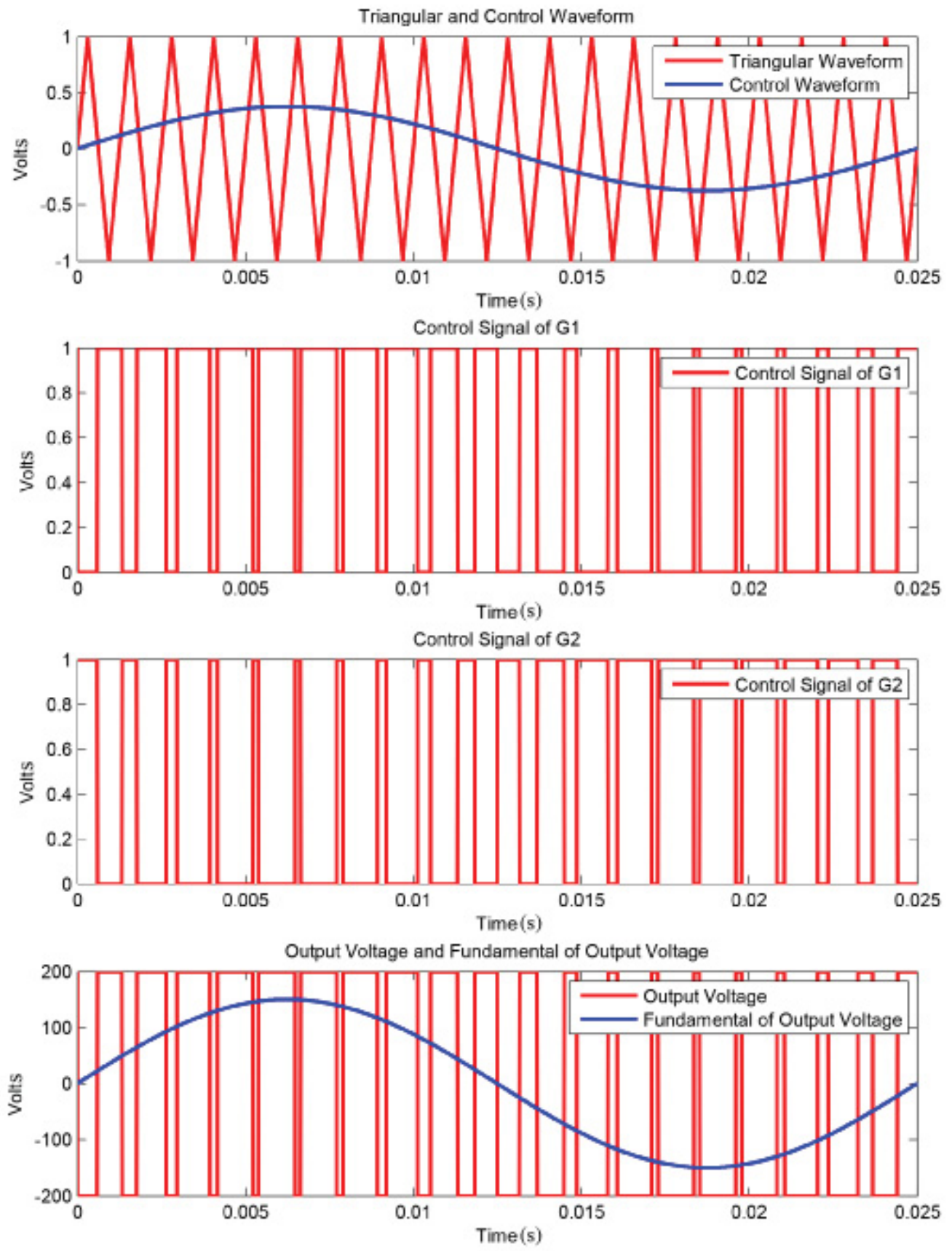

Figure 2-9: Single Phase PWM 

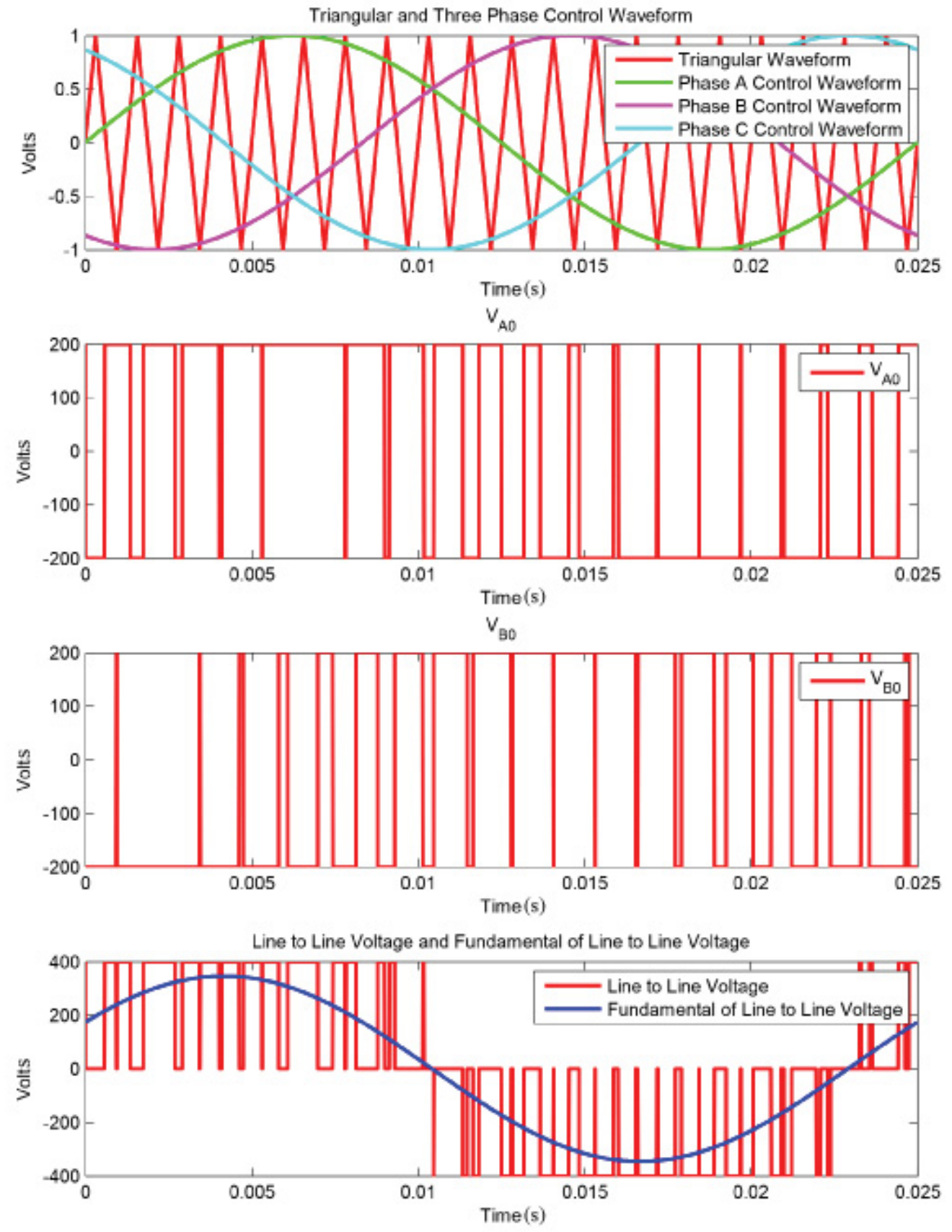

Figure 2-10: Three Phase PWM 


\subsection{Modulation Index}

Modulation Index (MI or $\left.m_{a}\right)$ is the ratio of the control signal $\left(V_{\text {control }}\right)$ and the peak of the triangular waveform $\left(\hat{V}_{t r i}\right)$ :

$$
m_{a}=\frac{V_{\text {control }}}{\hat{V}_{\text {tri }}}
$$

This quotient will help us calculate the peak of the fundamental voltage between

points $\mathrm{A}$ and $\mathrm{O}\left(\left(\hat{V}_{A O}\right)_{1}\right)$. Figure $2-10$ shows that the magnitude of the fundamental output voltage depends on the magnitude of the control signal, therefore we can write:

$$
\left(\hat{V}_{A O}\right)_{1}=\frac{V_{\text {control }}}{\hat{V}_{\text {tri }}} \frac{V_{d c}}{2}=m_{a} \frac{V_{d c}}{2}
$$

Based on the circuitry shown in Figure 2-8 we can deduce that:

$$
V_{A N}=V_{A O}+\frac{V_{d c}}{2}
$$

Since the difference between $V_{A N}$ and $V_{A O}$ is a DC offset, then their harmonic voltage components are the same and we can write:

$$
\left(\hat{V}_{A N}\right)_{h}=\left(\hat{V}_{A O}\right)_{h}
$$

In terms of the RMS values of the fundamental components of voltages we have:

$$
\begin{gathered}
\left(V_{L L}\right)_{1}(r m s)=\left[\left(V_{A N}\right)_{1}-\left(V_{B N}\right)_{1}\right](r m s) \\
{\left[\left(V_{A N}\right)_{1}-\left(V_{B N}\right)_{1}\right](r m s)=\frac{\sqrt{3}}{\sqrt{2}}\left(\hat{V}_{A N}\right)_{1}=\frac{\sqrt{3}}{\sqrt{2}}\left(\hat{V}_{A O}\right)_{1}=0.612 m_{a} V_{d c}}
\end{gathered}
$$




$$
\left(V_{L L}\right)_{1}(r m s)=0.612 m_{a} V_{d c}
$$

Based on Equation 2.19, if the DC bus voltage and MI are known, one can calculate the line to line voltage produced by the inverter. However, this equation is only valid when the inverter is operated in its linear region, when $0 \leq m_{a} \leq 1$. Above a value of 1 , the inverter will operate in the over modulation region. In this region, higher values of line to line voltage can be achieved. This is accomplished by increasing the peak value of the control signal above the peak value of the triangular waveform and essentially skipping the triangular pulses. This will continue until the control signal becomes so large that it is always above the triangular waveform. After this point, the inverter will enter a region of operation known as square wave operation which produces the highest value of line to line voltage but is independent of $m_{a}$. The $m_{a}$ at which the square wave operation starts depends on the fundamental frequency $\left(f_{1}\right)$ and the frequency of the triangular waveform or in other words the switching frequency $\left(f_{s}\right)$. The frequency modulation ratio $\left(m_{f}\right)$ is a quantity that can help understand this idea better and is defined as:

$$
m_{f}=\frac{f_{s}}{f_{1}}
$$

For example, the square wave operation for an $m_{f}$ of 15 starts at an $m_{a}$ of 3.24 . Figure 2-11 represents the behavior of the line to line voltage as a function of $m_{a}$ for an $m_{f}$ of $15[9]$. 


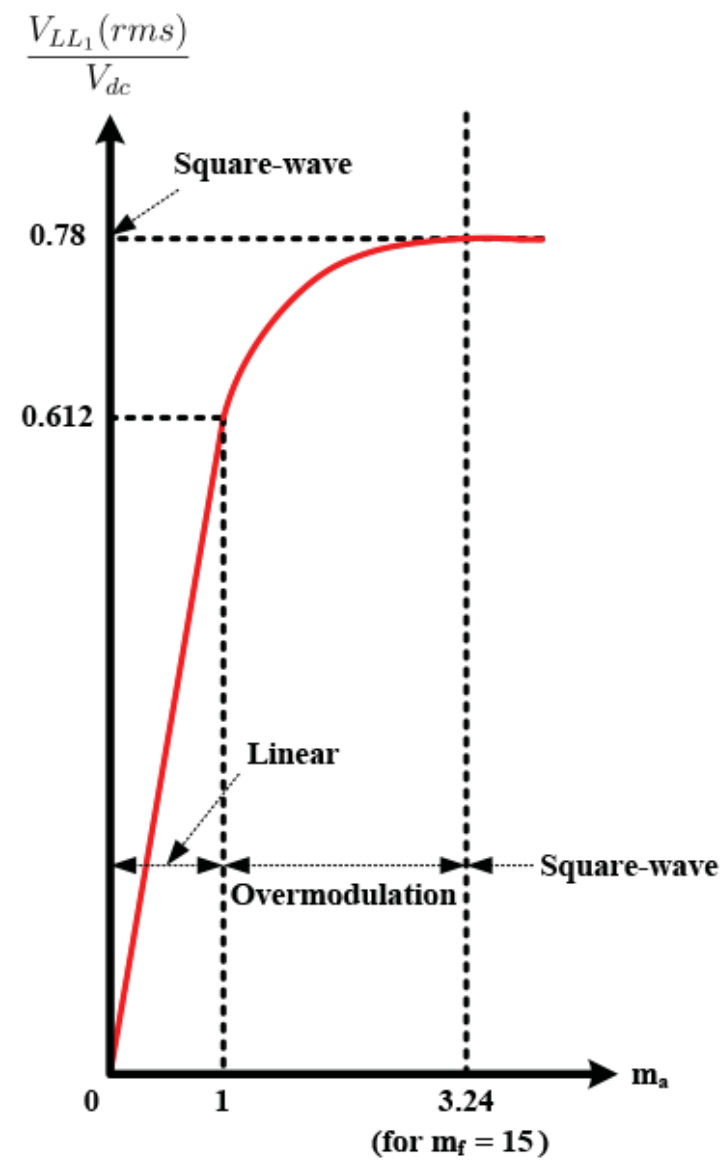

Figure 2-11: Normalized Line to Line Voltage vs. MI $[9]$

\subsection{Three-phase PWM waveforms and harmonic spectrum}

The harmonics produced by the inverter appear around frequency of $m_{f}$ and its multiples. Figure 2-12 shows the harmonic spectrum of the waveforms produced by an inverter when $m_{a}=0.8$ and $m_{f}=15$.

\subsection{Overall Control of the system with PWM}

Adding the PWM control to the previous control system explained in Section 2.4, will result in the full system in Figure 2-13. 


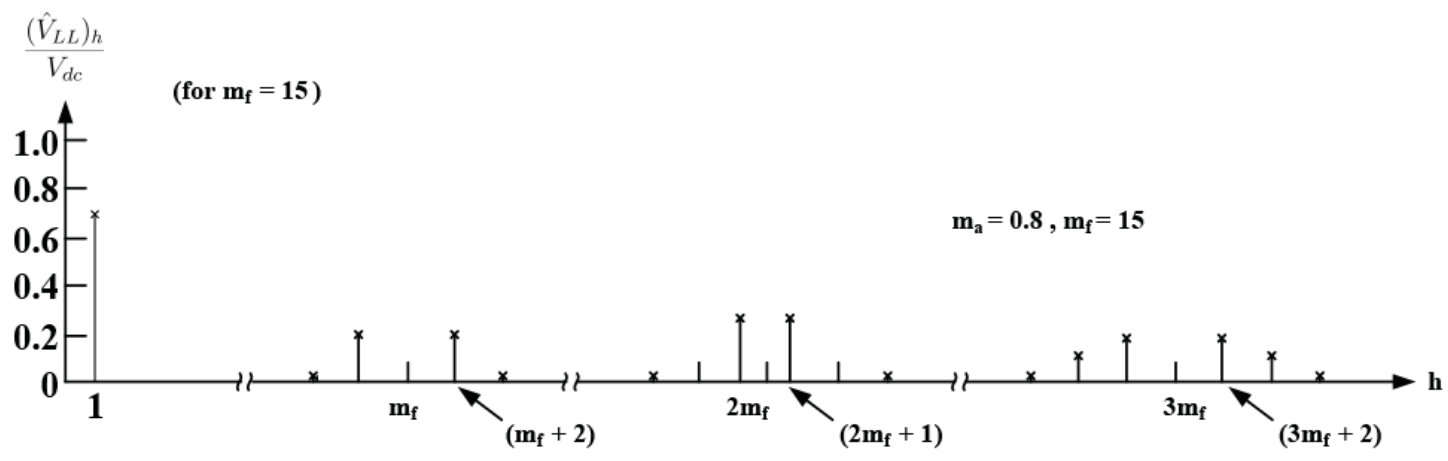

Figure 2-12: PWM harmonic spectrum

$[9]$

\subsection{Half Bridge DC-DC converter}

DC-DC converters are a family of power electronics topologies that can convert a source voltage to a desired voltage. A boost converter is one of these topologies, used to increase the output voltage level compared to the source voltage. Figure 2-14 demonstrates this topology.

The main idea behind the operation of this device is that when switch G2 is closed there will be current passing through the inductor and the voltage across the inductor will increase. During the same period, the load is supported by the capacitor voltage. The moment that G2 gets deactivated the diode D1 will conduct and the load will see the voltage of the source plus the voltage of the inductor. During this operation mode, the inductor will be drained and its energy will be transferred to the load and the capacitor. Therefore on average, the load will see a higher voltage output $\left(V_{\text {out }}\right)$ compared to the input voltage $\left(V_{\text {in }}\right)$. 

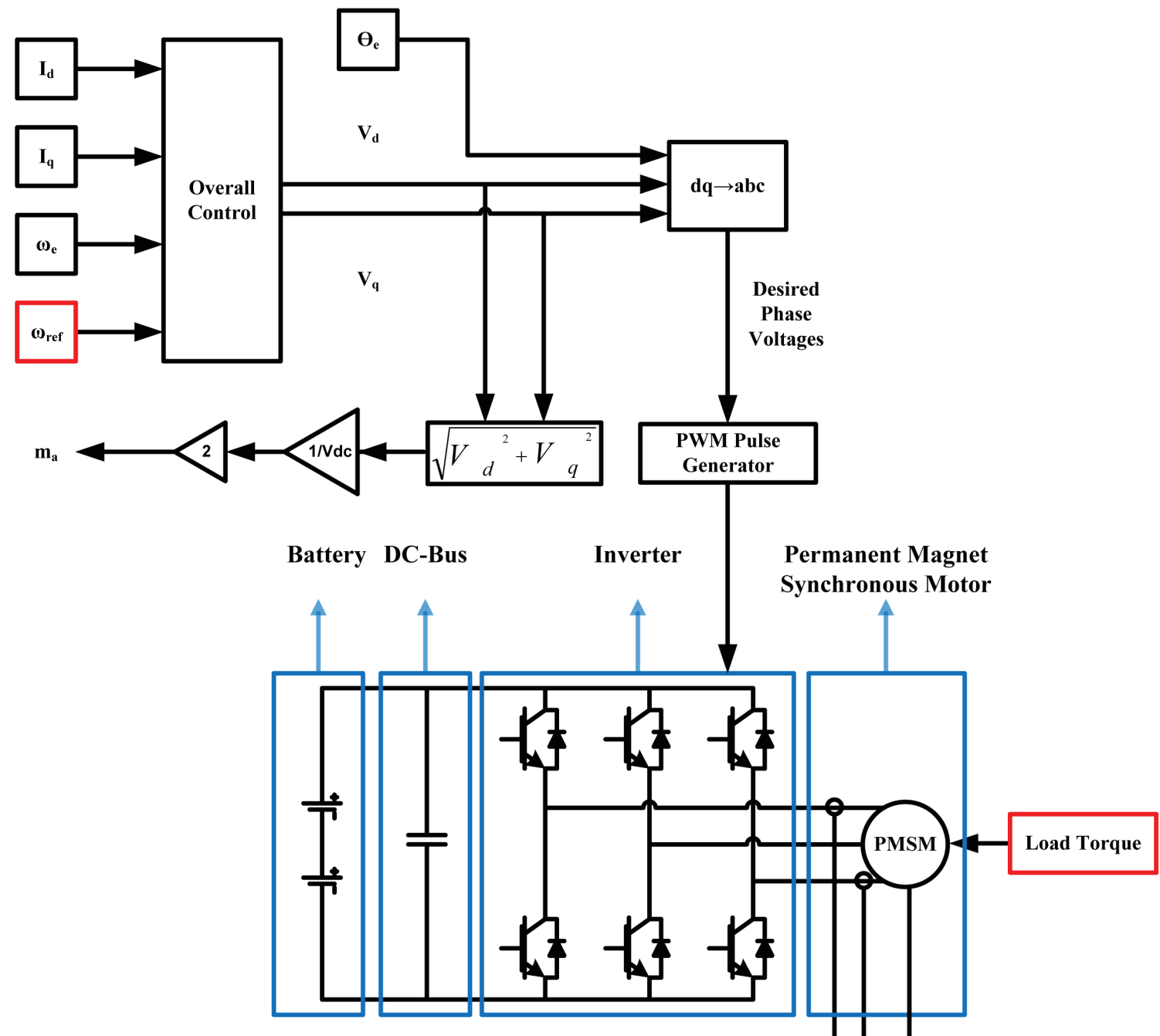

$i_{a}$

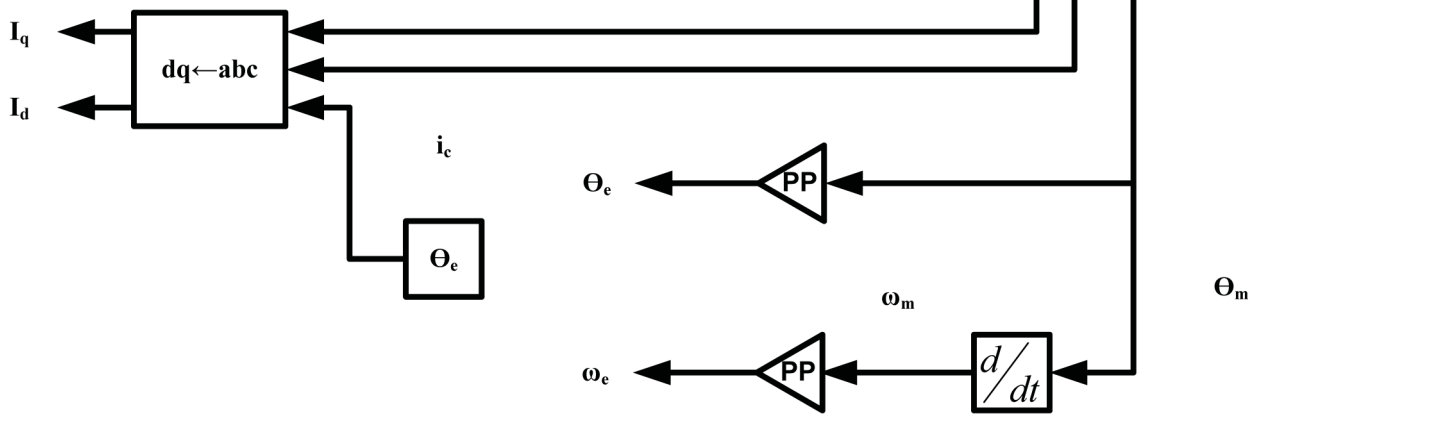

Figure 2-13: Overall System Control with PWM 


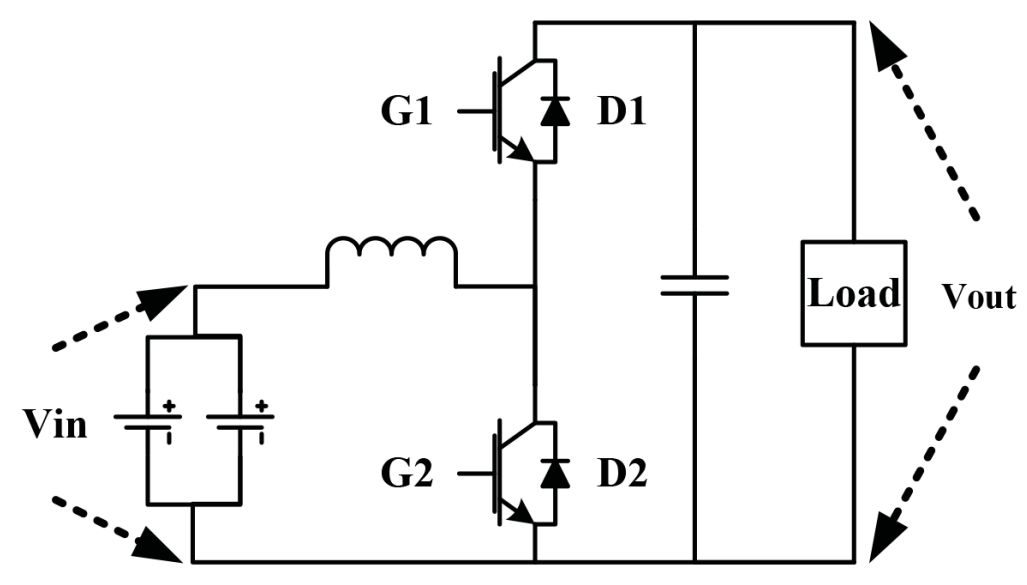

Figure 2-14: DC-DC Boost Converter

In each full cycle, the period that G2 is activated $\left(t_{o n}\right)$ compared to the duration of the whole cycle $(T)$ is called the duty cycle $(D)$. The duty cycle determines that output voltage. In order to implement this strategy, a control signal is compared to a sawtooth wave and whenever the control signal is higher than the sawtooth value, the transistor will be activated. In the reverse case, when the control signal is lower than the sawtooth value, the transistor will be deactivated [9]. The following two equations summarize the behavior of a boost converter.

$$
\begin{aligned}
D & =\frac{t_{\text {on }}}{T} \\
\frac{V_{\text {out }}}{V_{\text {in }}} & =\frac{1}{1-D}
\end{aligned}
$$

Figure 2-15 shows a sample operation point for a DC-DC converter with an input voltage of 400 volts and a duty cycle of $50 \%$. It can be seen that the voltage output is choppy but its average value is at the desired level. 

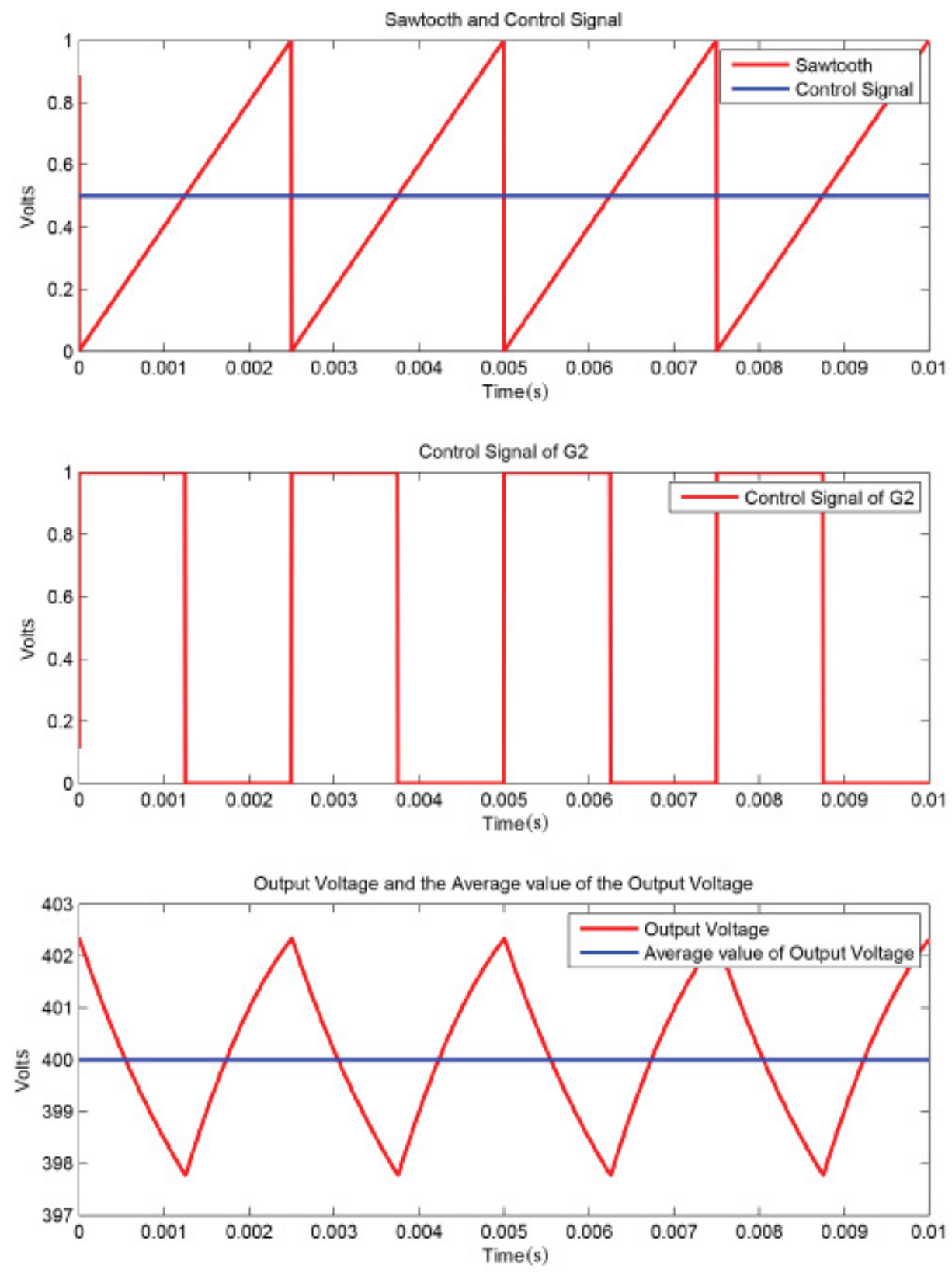

Figure 2-15: Sample operation for a boost converter 


\subsection{Quantification of Drive Cycles}

A drive cycle is the speed of the vehicle along with the driving time [1]. Different drive cycles can be used to represent the behavior of different vehicles under different conditions. Various metrics can be used to quantify vehicle drive cycles. For example, average and maximum velocities, acceleration rates, and grades, as well as location, kinetic intensity, temperature, road conditions, and many more. Average driving speed can be used to quantify driving cycles [11] and does not include the time spent stationary since this time does not significantly contribute to the energy consumption. The kinetic intensity is a metric which attempts to quantify the acceleration, velocity

and elevation effects of a drive cycle into a single metric [12]. The following equations show the derivation of the kinetic intensity.

$$
\begin{gathered}
\tilde{a}=\frac{\sum_{j=1}^{N-1} \operatorname{positive}\left(\frac{1}{2} \cdot\left(v_{j+1}^{2}-v_{j}^{2}\right)+g \cdot\left(h_{j+1}-h_{j}\right)\right)}{D} \\
v_{\text {aero }}^{2}=\frac{\sum_{j=1}^{N-1} \overline{v_{j, j+1}^{3}} \cdot \Delta t_{j, j+1}}{D} \\
k i \cong \frac{\tilde{a}=\frac{\tilde{a}}{v_{a e r o}^{2}}}{\sum_{j=1}^{N-1} \text { positive }\left(\frac{1}{2} \cdot\left(v_{j+1}^{2}-v_{j}^{2}\right)+g \cdot\left(h_{j+1}-h_{j}\right)\right)} \\
\sum_{j=1}^{N-1} \frac{v_{j, j+1}^{3}}{D} \cdot \Delta t_{j, j+1}
\end{gathered}
$$


Where $k i$ is kinetic intensity, $\tilde{a}$ is the characteristic acceleration, $v_{\text {aero }}$ is the aerodynamic speed, $v$ is the vehicle velocity, $h$ is the height above a certain reference, $\Delta t$ is the time step size, $g$ is the acceleration due to gravity and $D$ is the drive cycle distance. The positive notation means that only positive values are considered and the negative values are neglected. Based on these equations, a drive cycle with high acceleration rates and low speeds will have a high kinetic intensity. 


\section{Chapter 3 Literature Review}

In this section, some of the relevant previous work done in this topic is represented. At the beginning, the main idea of the project was to study advance powertrain topologies that meet the following criteria:

1. A drive topology that could be beneficial for delivery truck drive cycles, which implies that the drivetrain has to have high efficiencies at low RPMs, and offer a superior performance for drive cycles with high kinetic intensity.

2. A drive topology that can be implemented by simple modification of classical systems, without a huge deviation from the traditional drives.

In the preliminary literature review, it became clear that a drive topology that uses a bidirectional DC-DC converter between the battery and the inverter could meet the mentioned criteria. These discoveries will be explored in the next sections. Furthermore it was decided to put the focus on bidirectional DC-DC converters that use a half bridge boost converter topology for simplification. The following are some of the applications for the bidirectional DC-DC converters for automotive use.

\subsection{Linkage of Energy Storage and Energy Conversion Systems (ESECS)}

There are many different energy sources and energy conversion systems with dif-

ferent advantages and disadvantages that can be used for powering electric vehicles. Some of these sources include batteries, Ultra Capacitors (UC), Photovoltaic Cells (PVC), Internal Combustion Engines (ICE) with Electric Generators, and Fuel Cells 
(FC). The voltage of different ESECS can be adjusted to a desired value, and power from all these sources can be distributed to a DC bus and passed to an inverter. Using multiple ESECS in the same system provides the freedom to benefit from different advantages of various ESECS and provides more controllability. The Toyota Prius, one of the most well-known hybrid vehicles, uses a DC-DC converter to boost the battery voltage from a $200 \mathrm{~V}$ battery to a $500 \mathrm{~V}$ DC bus, which is shared between the main inverter and the generator's inverter. In addition to greater controllability, the torque-speed envelope of the motor can be expanded when the inverter is operated at a higher DC voltage [13-16].

Of course, these systems can become more elaborate. For example, [17] studies a system that uses three types of ESECS which are batteries, FCs and UCs. These are connected to the main DC bus via three DC-DC converters. UCs have a very fast transient response and high power density. This ESECS can easily go through a fast discharge and charge cycle and therefore is ideal for accelerations and deceleration for regenerative braking. However, UCs have a low energy density and for low power and steady operation, the batteries will be the main source of energy since they are most efficient for these operation conditions. Finally, FCs which have low efficiencies at low powers and transient operations, can be used at high and steadystate operation points. Control, implementation, efficiency, power management, and possible performance advantages of drivetrains with DC-DC converters are explored in several studies [18-25]. 


\subsection{Increase of the efficiency in certain regions of operation}

Another way that the DC-DC converters can be used for traction applications is to increase the system efficiency in certain operation regions. This is the main focus of this project. Exploring this idea has led to 4 phases of this project which build upon one another.

\subsubsection{Phase 1}

In order to properly understand this specific application, the operation of a traditional system, which is composed of a battery and a PWM operated 2 level inverter and a PMSM, has to be explored. In a traditional system, the speed of a PMSM is increased by increasing the phase voltage of the motor. This is done by increasing the MI of the inverter. An increase in MI will increase the magnitude of the three phase voltages. However, in order to increase the motor's RPM the electrical angular frequency $\left(\omega_{e}\right)$ should also be increase proportionally to the mechanical angular frequency $\left(\omega_{m}\right)$. At low RPMs the MI is increased linearly until the base speed and the rated phase voltage of the motor is achieved. After that point the MI is kept at a constant value and the phase voltage is maintained at its rated value and the motor will operate in its constant power region. This control strategy is shown in Figure 3-1.

Unfortunately, inverters are less efficient and produce more current harmonics when they are operated at low MIs. An increase in the current harmonics results in more copper losses and torque ripples of the motor. Therefore, it would be ideal to be able to operate the inverter at higher MIs for a wider range of the RPMs. This idea can be achieved by using a lower voltage battery and a boost converter, as in 


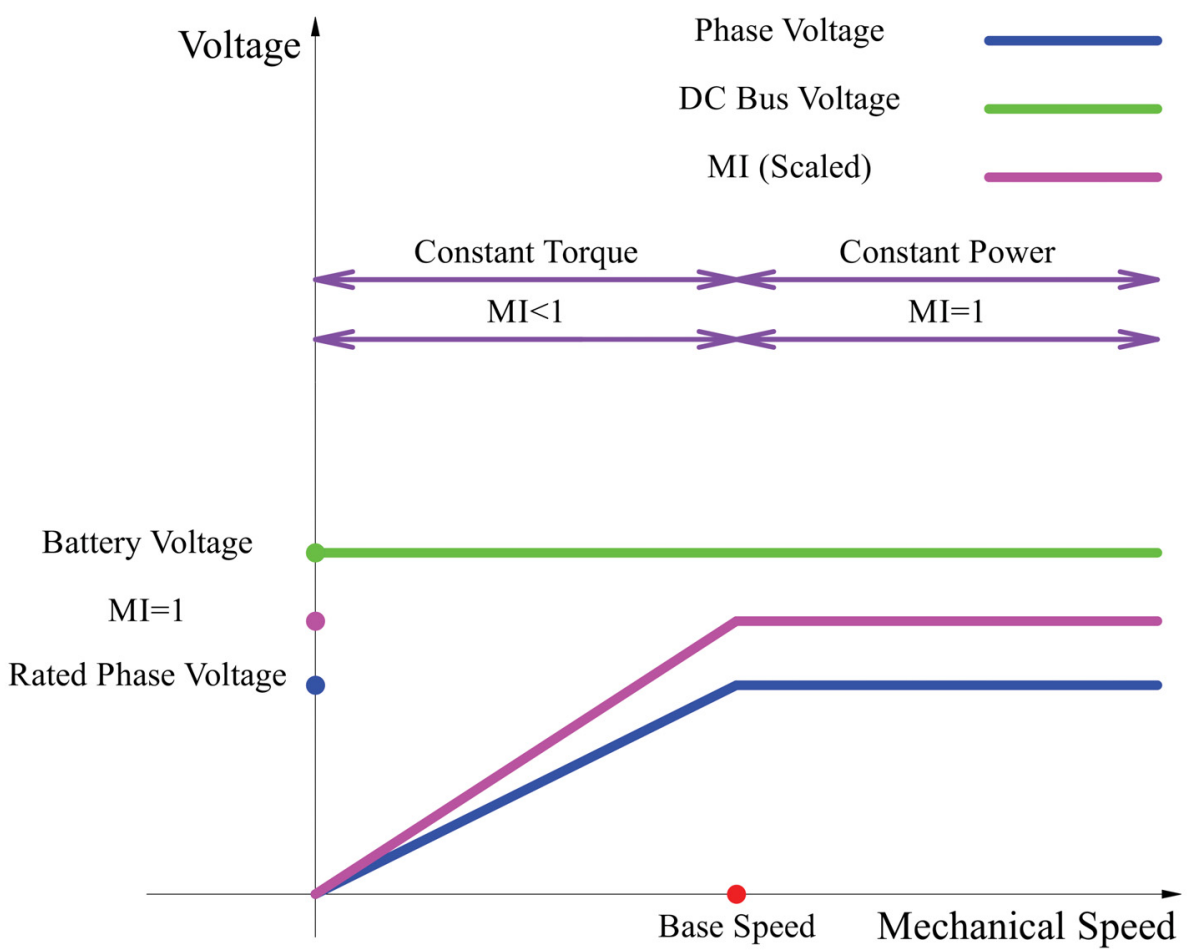

Figure 3-1: Control Strategy for traditional PWM controlled motor

system S2. In this system at low RPMs, the MI is ramped up quickly until the value of ' 1 ' is achieved. At this point, the MI is kept at ' 1 ' and the DC-DC converter starts to increase the DC bus voltage until the rated phase voltage and the base speed are achieved. After this point, the system will operate in the constant power region $[26,27]$. This control strategy is demonstrated in Figure 3-2.

Both systems provide the same fundamental component of phase voltage at every operating point of the motor. The only difference is that the inverter produces a less distorted waveform before the base speed in S2. It can be seen that in S2, the MI of ' 1 ' is maintained for a wider range of operation. This region is where improvement is introduced. 


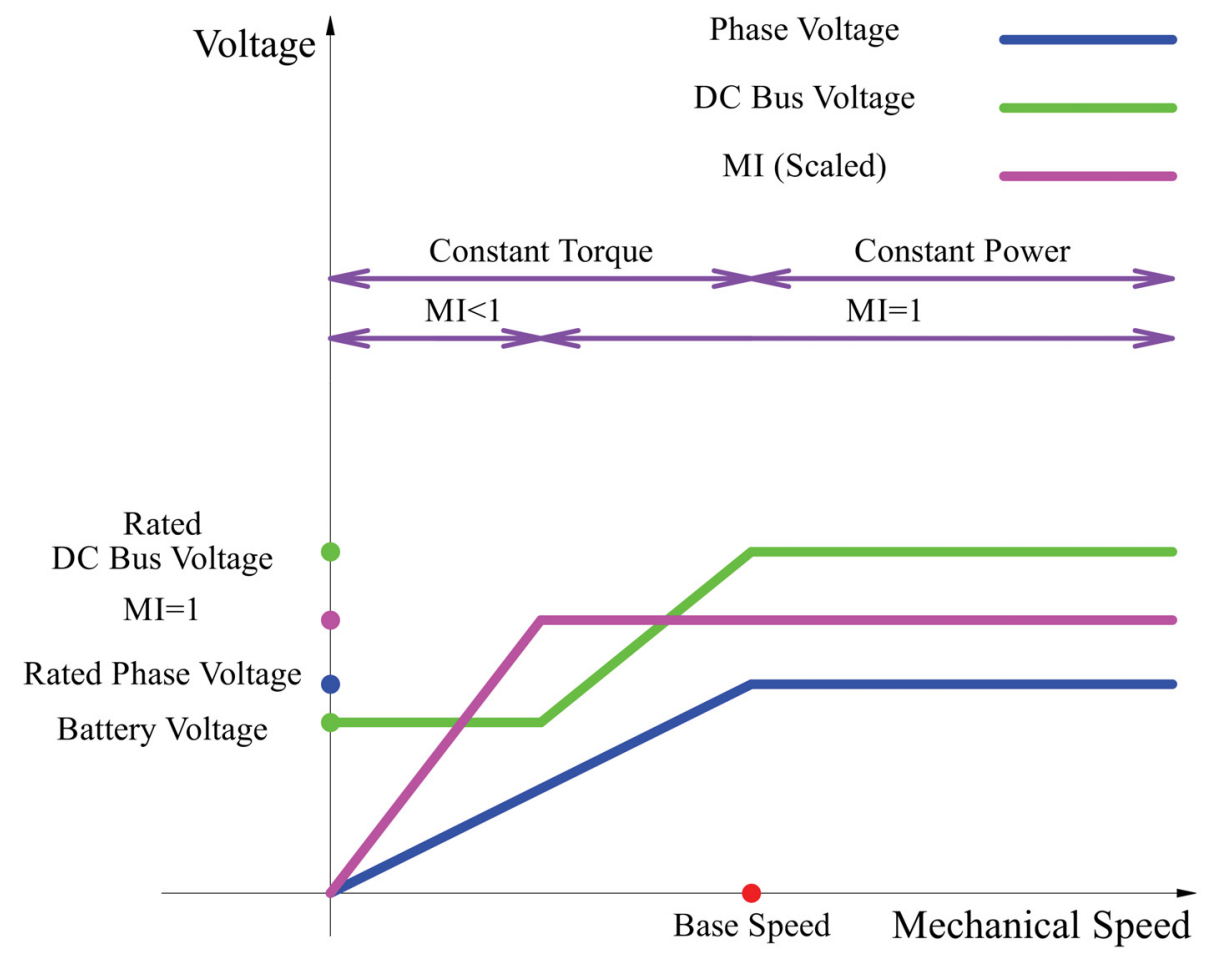

Figure 3-2: Variable DC Bus Voltage Control with Target MI of '1'

The benefits of $\mathrm{S} 2$ can be summarized by the following two points:

1. The inverter is more efficient and produces less distorted waveforms before the base speed. The decrease in the current harmonics will reduce the motor's copper losses. Therefore, the overall system becomes more efficient at low RPMs [26-31].

2. Due to the decrease in current harmonics, the torque ripples are reduced in the low RPM range and the user experience is improved [26, 27,32].

Due to the fact that the system is more beneficial at low RPMs, it becomes a desirable solution for vehicles that go through lots of start and stop cycles and operate at low RPMs. Delivery trucks meet this profile [11]. Furthermore, the 
traditional system does not need to be altered significantly since the new system is created by the introduction of a DC-DC converter. As previously mentioned, these two characteristics are part of the original criteria of this project. The two main disadvantages of system S2 are:

1. Increase in cost and complexity with a reduction in reliability [13].

2. Additional losses in the DC-DC converter [13].

Even though the DC-DC converter losses have been introduced in the system, the increase in the efficiency of the motor and the inverter allow the overall system to be more efficient in the low RPM range. The first part of this project is to explore the application of the topology for electric medium duty delivery trucks. The primary goal is to examine this system in the context of an appropriate drive cycle for delivery trucks. One of the drive cycles that models the behavior of a delivery truck very well is the Orange County Cycle (OCC) $[11,33]$ which is used for this phase of the project.

\subsubsection{Phase 2}

The next step in studying this topology was to examine the effectiveness of the system for different drive cycles that can accurately represent the behavior of a delivery truck, and discover if the system is more beneficial for some than others. [11] demonstrates that some other cycles such as the New York City Cycle (NYCC) and HTUF-4 class 4 cycle (short version) are also applicable to delivery trucks. Two

additional cycles, the common Urban Dynamometer Driving Schedule (UDDS [1]) and the Heavy Duty UDDS (UDDSHD [34]) are also used for comparison purposes. This idea was further explored by the collection of actual delivery routes taken by a 
Purolator truck in the greater Montreal area. In total, this phase of the study uses 10 different drive cycles to explore the system performance in various conditions.

\subsubsection{Phase 3}

As mentioned, the PWM control strategy in the linear region varies the MI from zero to 1 in order to achieve different phase voltages. However, it is possible to operate an inverter in the overmodulation region which is not linear(Figure 2-11). Therefore, instead of using a target value of ' 1 ' for the MI, another value can be chosen. Figure 3-2 demonstrates this control strategy for a target MI of '1.5'.

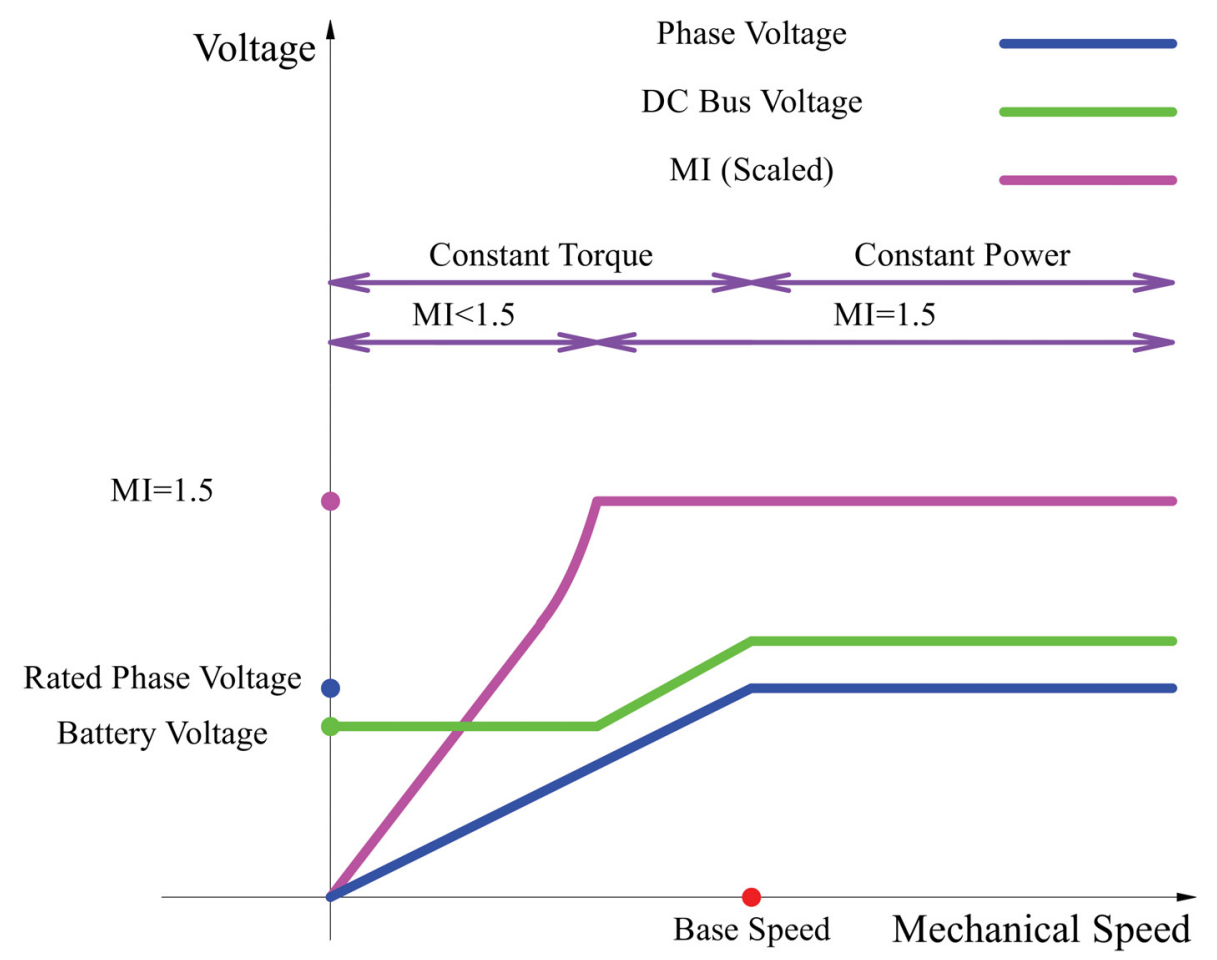

Figure 3-3: Variable DC Bus Voltage Control with Target MI of '1.5'

In most publications, it is mentioned that the target MI has to be high (i.e. close to ' 1 ') for the reduction of the harmonics, but none of them used the target MI 
as an optimization parameter. An interesting point about the overmodulation region is the fact that there are more sideband harmonics around the harmonic frequencies and their multiples. However, the dominant harmonics may not be as large as in the linear region. Depending on the nature of the load and the switching frequency, the overall system could be even more efficient compared to the linear region [9]. Changing the target MI also affects the amount of boost that the DC-DC converter needs to provide, which changes its efficiency map. Since there was no substantial previous work dealing with the target MI, it was decided to treat this value as an optimization parameter and study its effect on the energy consumption of the truck through the appropriate drive cycles.

\subsubsection{Phase 4}

The effect of the battery voltage is another parameter that was not studied in previous publications. A lower battery voltage will increase the region of operation where the MI can be '1' or above, which, based on the theory, will cause the inverter to be more efficient for a larger RPM range. However, a lower battery voltage requires that the DC-DC converter boosts the voltage more and consequently increase the DC-DC converter losses. Therefore, in this phase of the project the battery voltage and the target MI were both used as optimization parameters and the effectiveness of the system was studied in the context of different drive cycles. 


\section{Chapter 4 Methodology for Simulation}

\subsection{Phase 1}

\subsubsection{Baseline, S1}

The first step in this project was characterizing the baseline system S1. The overall control strategy of the system that was explained in Section 2.4 was implemented in MATLAB and Simulink. Figures 4-1 \& 4-2 show the representation of the system in Simulink. The losses in power electronics and the motor were respectively implemented in PSIM and MotorSolve. Figure 4-3 shows the inverter model in PSIM. The important information that was collected were the efficiency map of the system and the THD map of the motor. This was done by operating the system at different torque and speed set points and examining the output of the simulated system. In order to make sure that the system had reached the steady state, a simulation time of 1 second was chosen. The motor angle, motor speed and motor torque as a function of time for a target set point of $100 \mathrm{Nm}$ and 10000 RPM are shown in Figure 4-4. The motor angle is constantly increasing throughout the simulation, but the motor speed increases at the beginning until the target speed is reached and stays at 1000 RPM. At the beginning of the simulation, the average value of the motor torque is at its peak value of $170 \mathrm{Nm}$ and when the target speed is reached it reaches the target value of $100 \mathrm{Nm}$. Similarly, for the same operation point the input and output power

of the motor and the inverter losses are shown in Figure 4-5. It can be seen that 
at the beginning, these parameters vary until they reach their steady state value. The THD of the motor currents are calculated based on the average THD of the three phase currents. For the same operation, point a sample waveform of the phase currents is shown in Figure 4-6. At the beginning of the simulation, when the motor is producing peak torque, the amplitude of the motor current is at its highest value. Once the steady state is reached and the peak torque is not needed, the peak value of the current drops to a lower value. Figure 4-7 shows the steady-state waveforms of the same currents. It can be seen that the currents do not have a pure sinusoidal shape due the fact that the motor is fed by an inverter which is not an ideal sinusoidal voltage source. 
Discrete,

$\mathrm{T} s=\mathrm{T} s s$

powergui

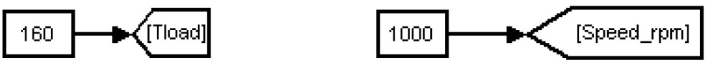

Load Torque and Reference Speed
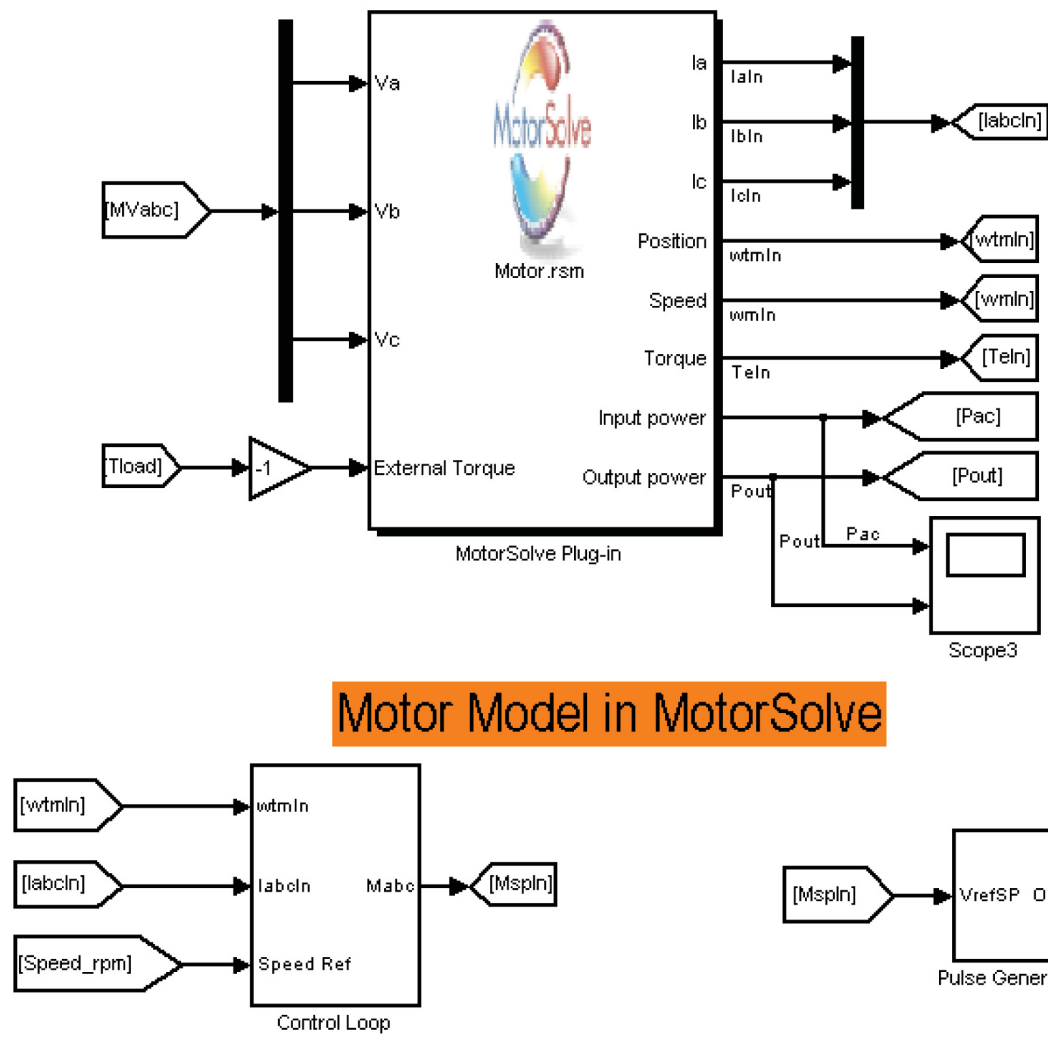

Overall Control with Flux-weakening

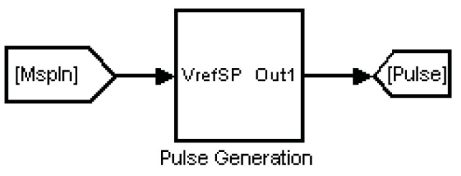

Firing Pulse Generation

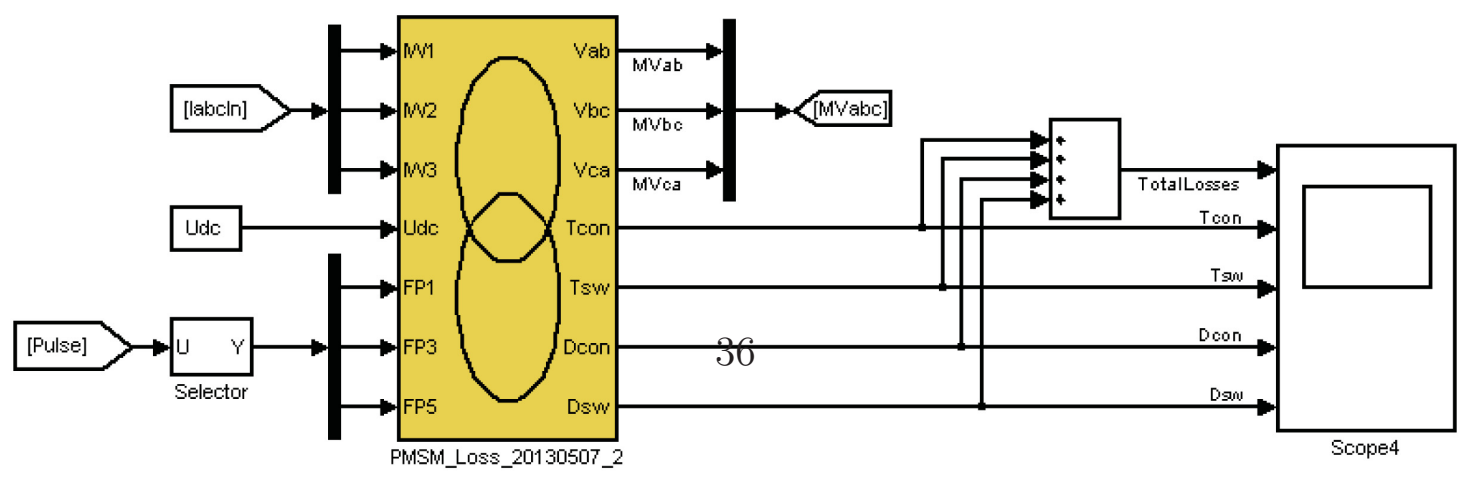

Power Electronics Circuit in PSIM

Figure 4-1: Overall System Implementation in Simulink 

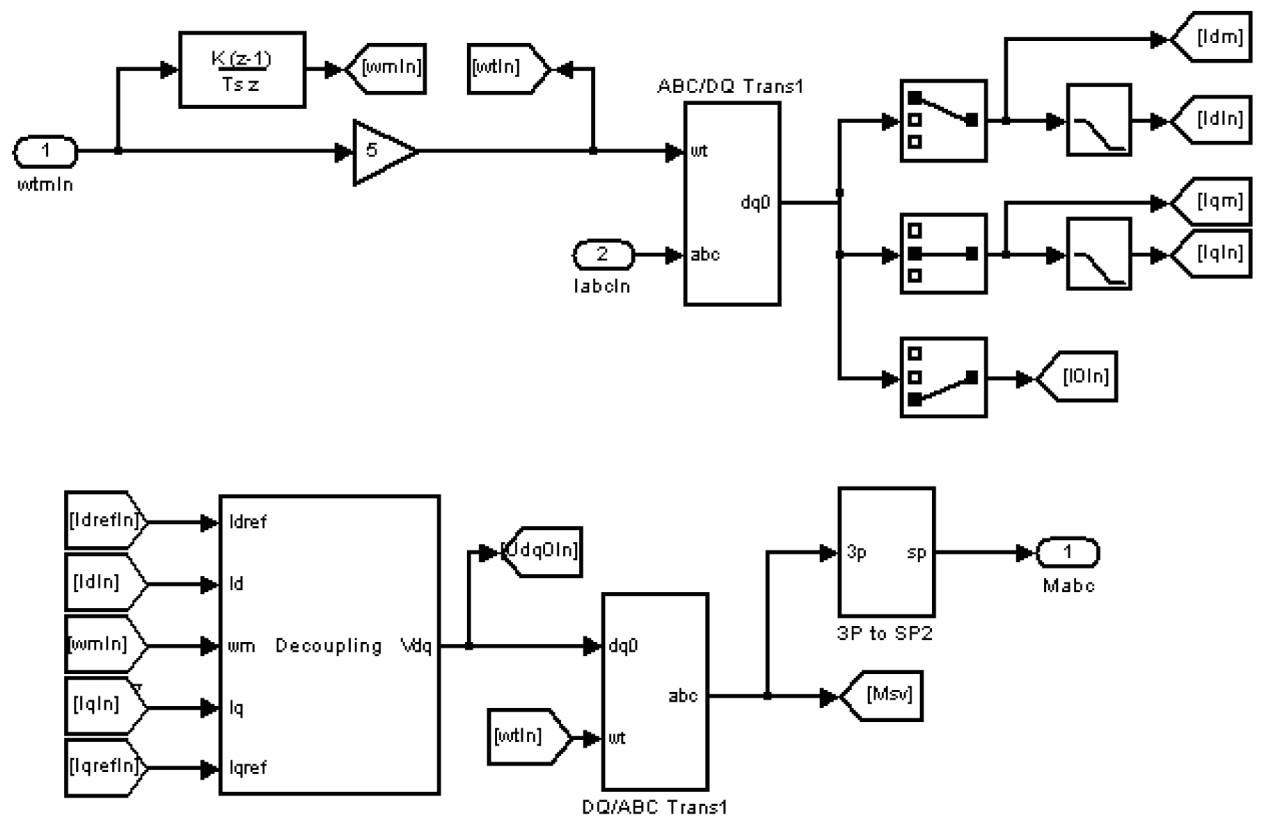

\section{Decoupling}
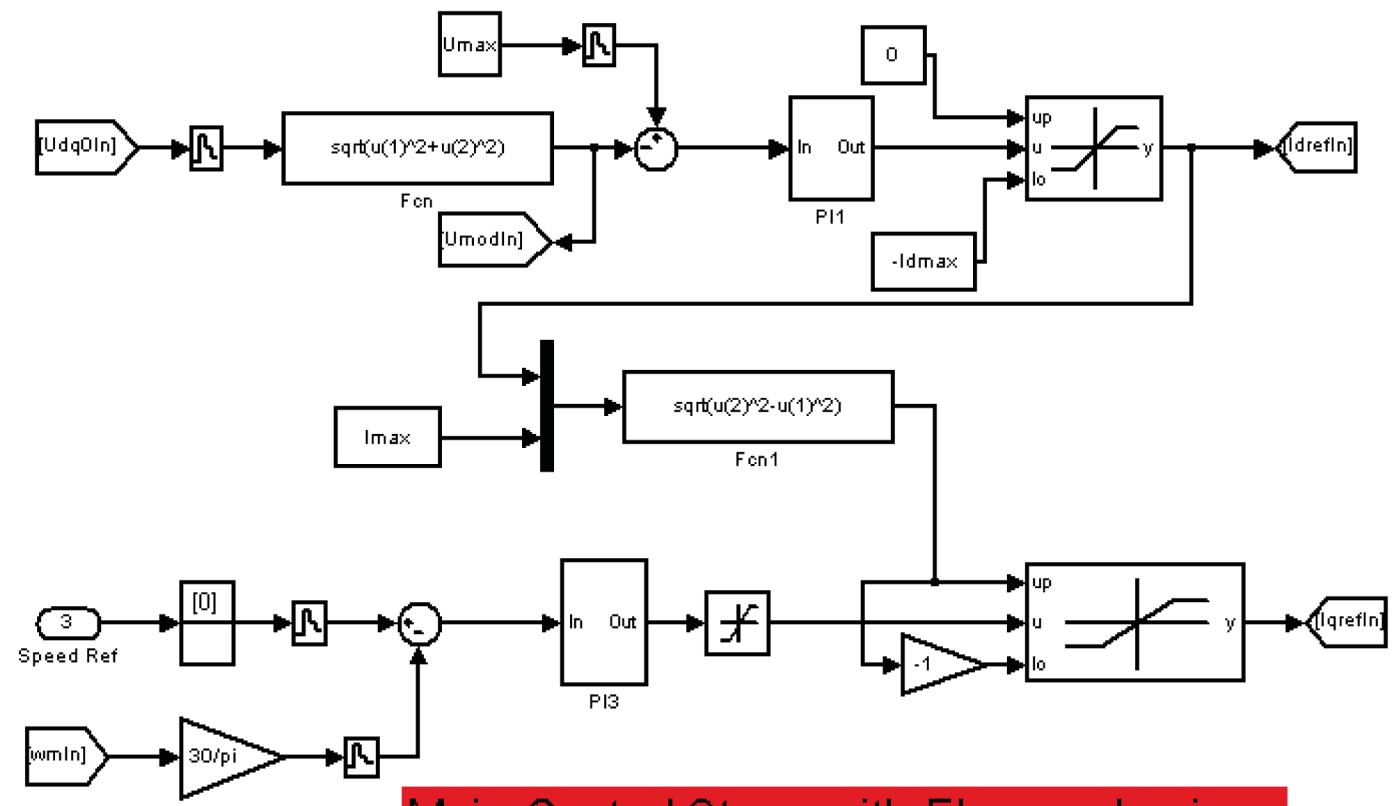

\section{Main Control Stage with Flux-weakening}

Figure 4-2: Overall Control with Flux Weakening in Simulink 


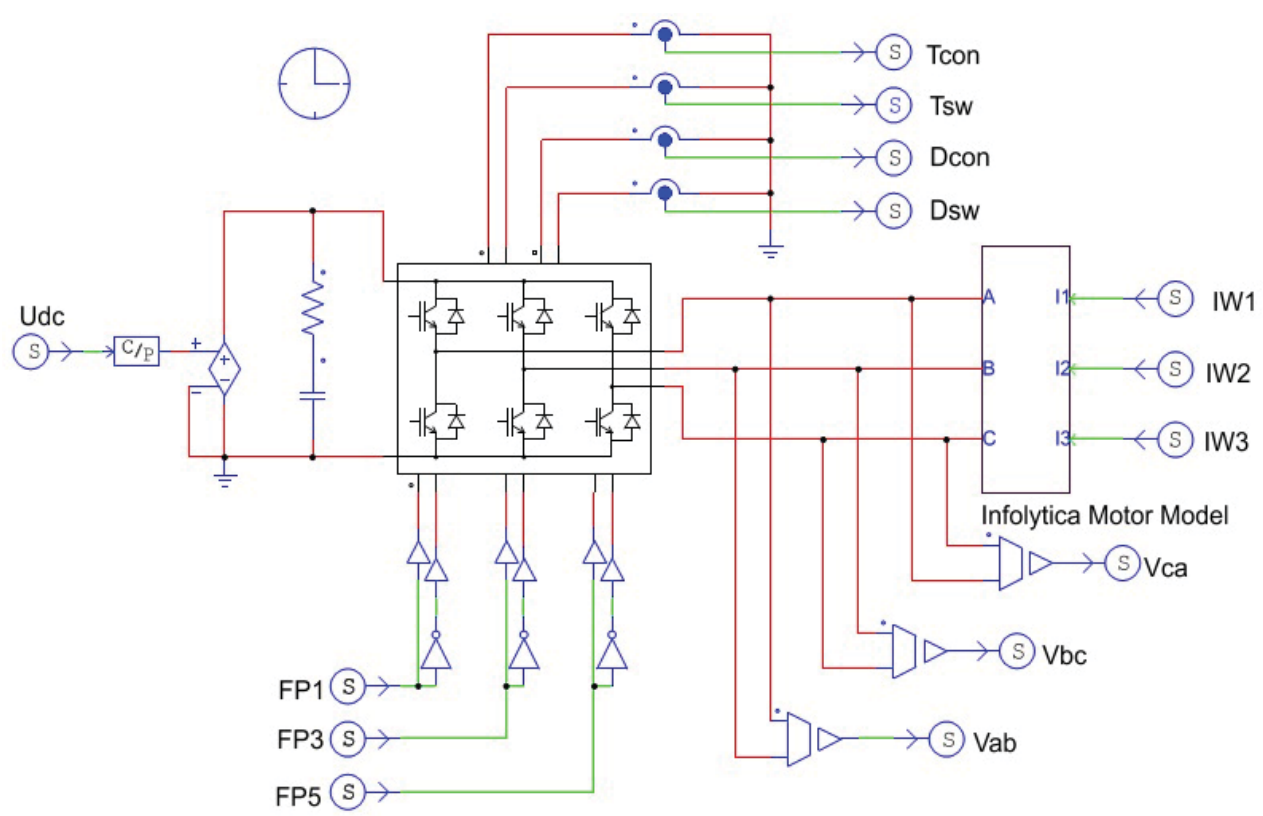

Figure 4-3: Inverter Model in PSIM

The efficiency of the motor $(M E)$ was calculated as:

$$
M E=100 \times \frac{P_{M O T_{-} O U T}}{P_{M O T_{-} I N}}
$$

Where, $P_{\text {MOT_out }}$ is the output power of the motor and $P_{M O T \_I N}$ is the input power of the motor. The efficiency of the inverter $(I E)$ was calculated as:

$$
I E=100 \times \frac{P_{M O T \_I N}}{P_{M O T_{-} I N}+P_{I N N_{\_} L O S S E S}}
$$

Where, $P_{I N V \_L O S S E S}$ is the inverter losses. The overall system efficiency (SE) was calculated as:

$$
S E=M E \times I E
$$



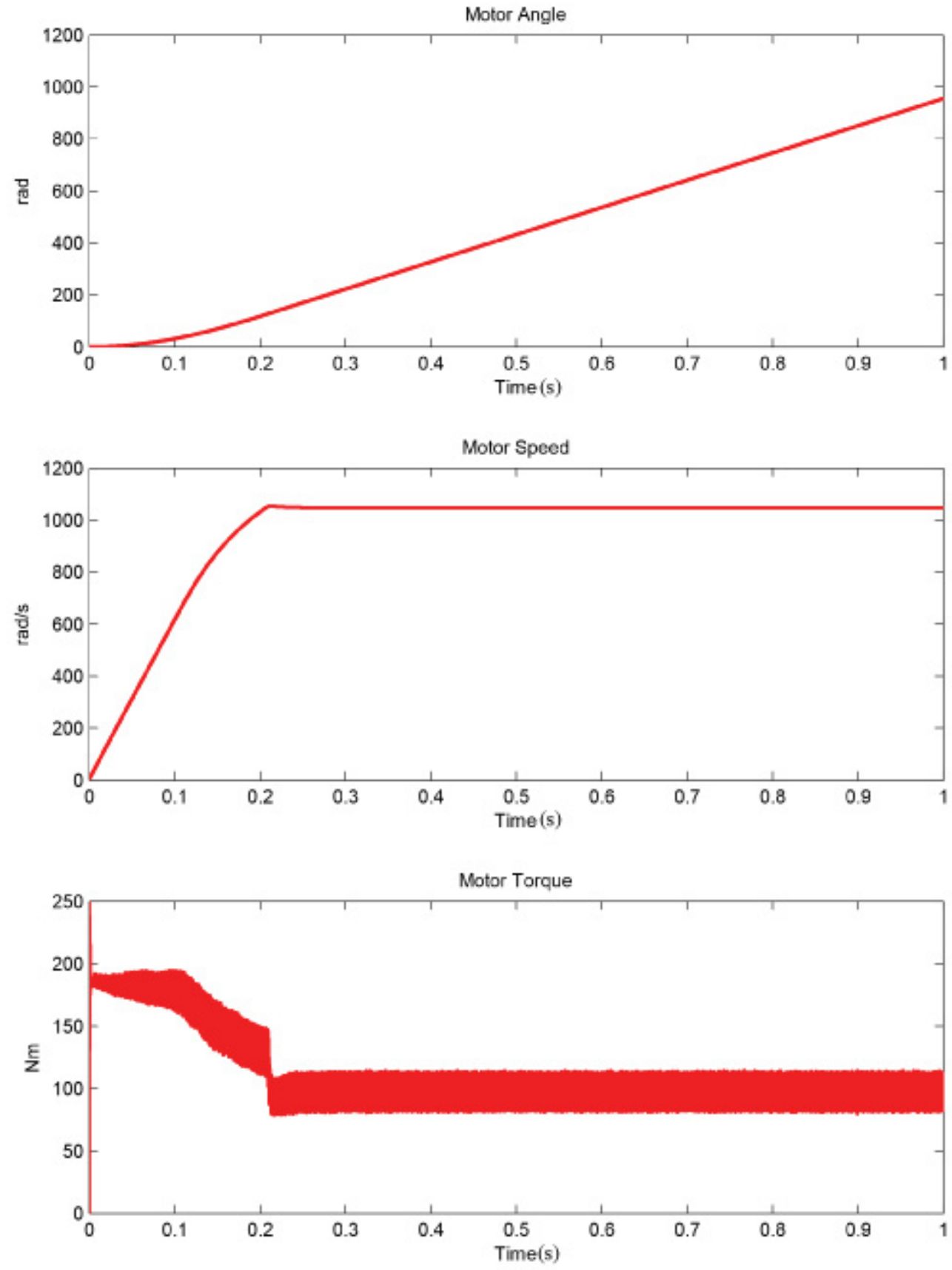

Figure 4-4: Motor angle, speed and torque for operation point of 100 NM and 1000 $\mathrm{RPM}$ 

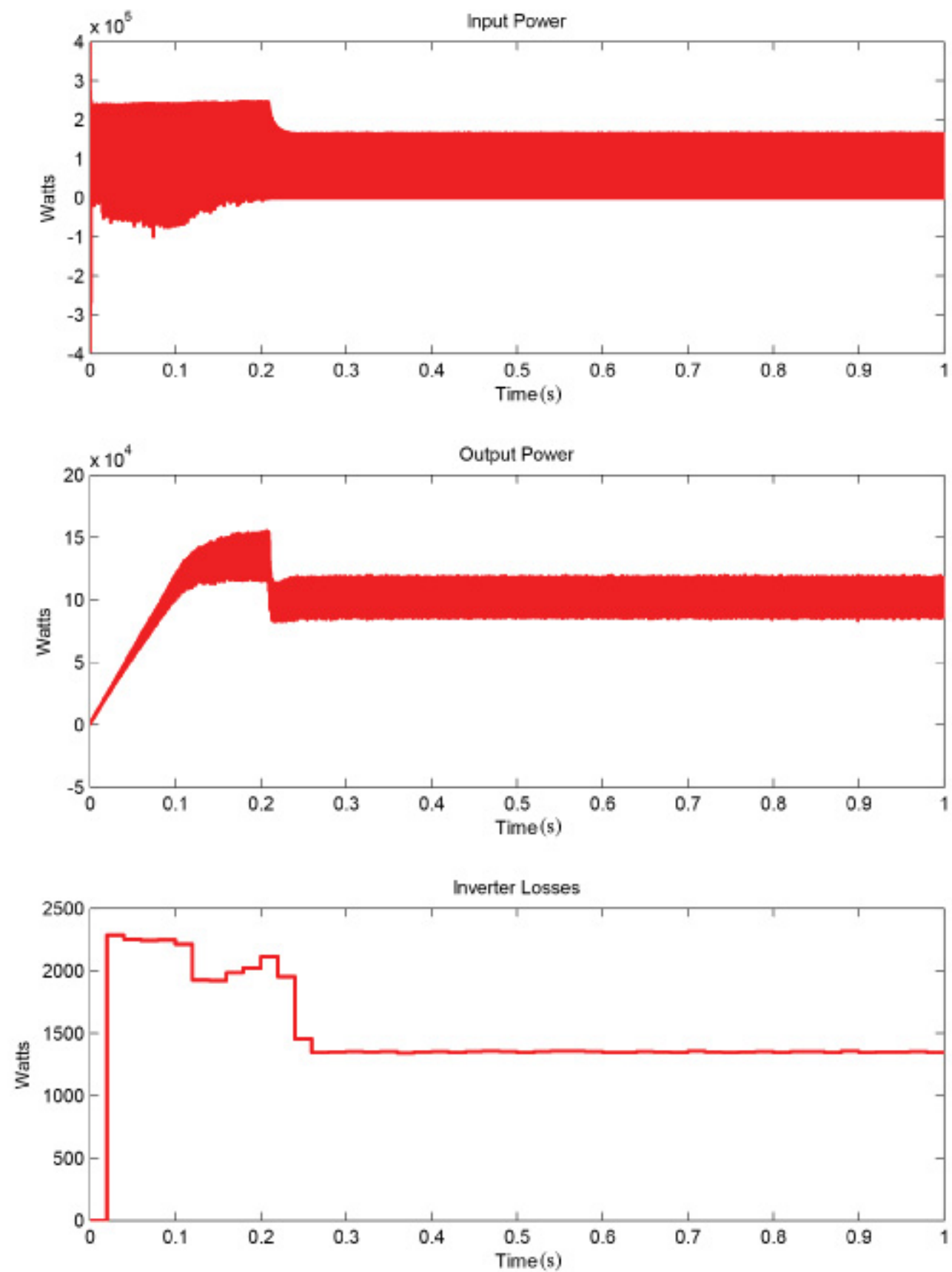

Figure 4-5: Motor input and output power and the inverter's losses 

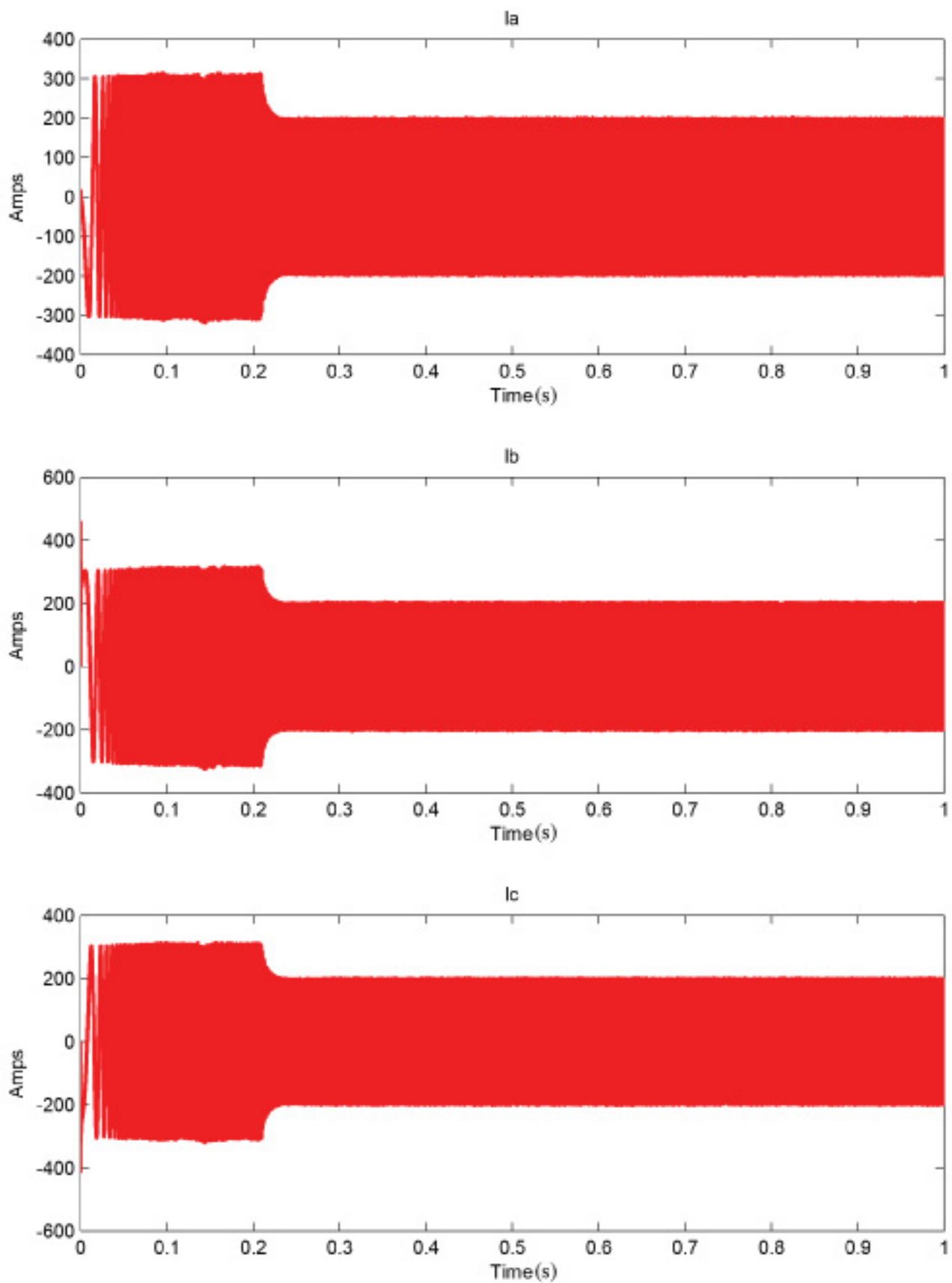

Figure 4-6: Motor Currents 

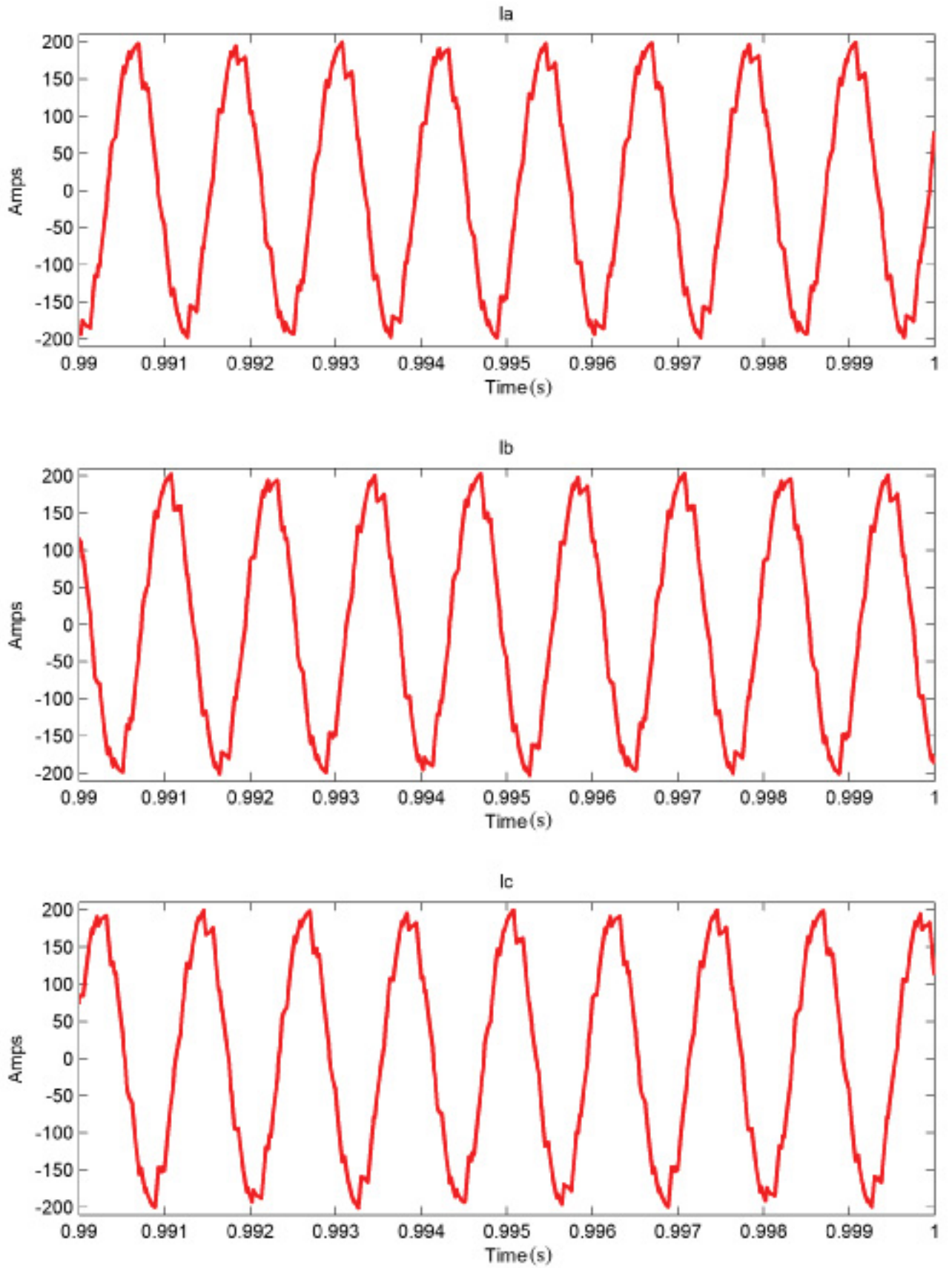

Figure 4-7: Motor Currents in steady-state 
Once the complete efficiency maps of the motor and inverter were obtained, the global efficiency map was created. The next step was to look at the energy use of the system during an OCC cycle. A package called AVL Cruise was used for the simulation of energy consumption over a cycle. This program uses the vehicle parameters to calculate the energy needed for the vehicle to follow the speed trace of a drive cycle. The software considers losses due to aerodynamic drag and rolling friction. Furthermore, the global efficiency map is imported in AVL Cruise in order to simulate the powertrain losses through the cycle. The vehicle architecture is shown in Figure 4-8. It can be seen that each rear wheel is operated by a separate motor. A single final gear ratio gearbox is used for each motor. The battery voltage is 460 $\mathrm{V}$ and regenerative braking is not considered. Table 4-1 summarizes some of the important vehicle parameters.

Table 4-1: Simulation Parameters

\begin{tabular}{lllll}
\hline \multicolumn{2}{c}{ Motor Parameters } & & \multicolumn{2}{c}{ Vehicle Parameters } \\
\cline { 1 - 2 } \cline { 5 - 6 } Base Speed & $6,000 \mathrm{RPM}$ & & Mass & $5445 \mathrm{~kg}$ \\
Max Speed & $12,000 \mathrm{RPM}$ & & Frontal Area & $7.0 \mathrm{~m}^{2}$ \\
Power & $107 \mathrm{~kW}$ & & Drag Coefficient & 0.7 \\
Torque & $170 \mathrm{Nm}$ & & Wheel Diameter & $0.393 \mathrm{~m}$ \\
\hline
\end{tabular}

The vehicle parameters are based on trucks used by the APC's industrial partner; Purolator. The motor parameters come from one of the electric machines used in the APC project. This specific motor was chosen since with a final drive ratio of 17.25 , it will provide a maximum speed of just over $100 \mathrm{~km} / \mathrm{h}$ and a maximum gradability of nearly $30 \%$ to the designed truck. The U. S. Postal Service uses a gradability of $25 \%$ for its wost case scenario [35] and according to Purolator for fuel economy reasons a 
top speed less than $100 \mathrm{~km} / \mathrm{h}$ is preferred. Therefore, the vehicle design meets these two requirements.

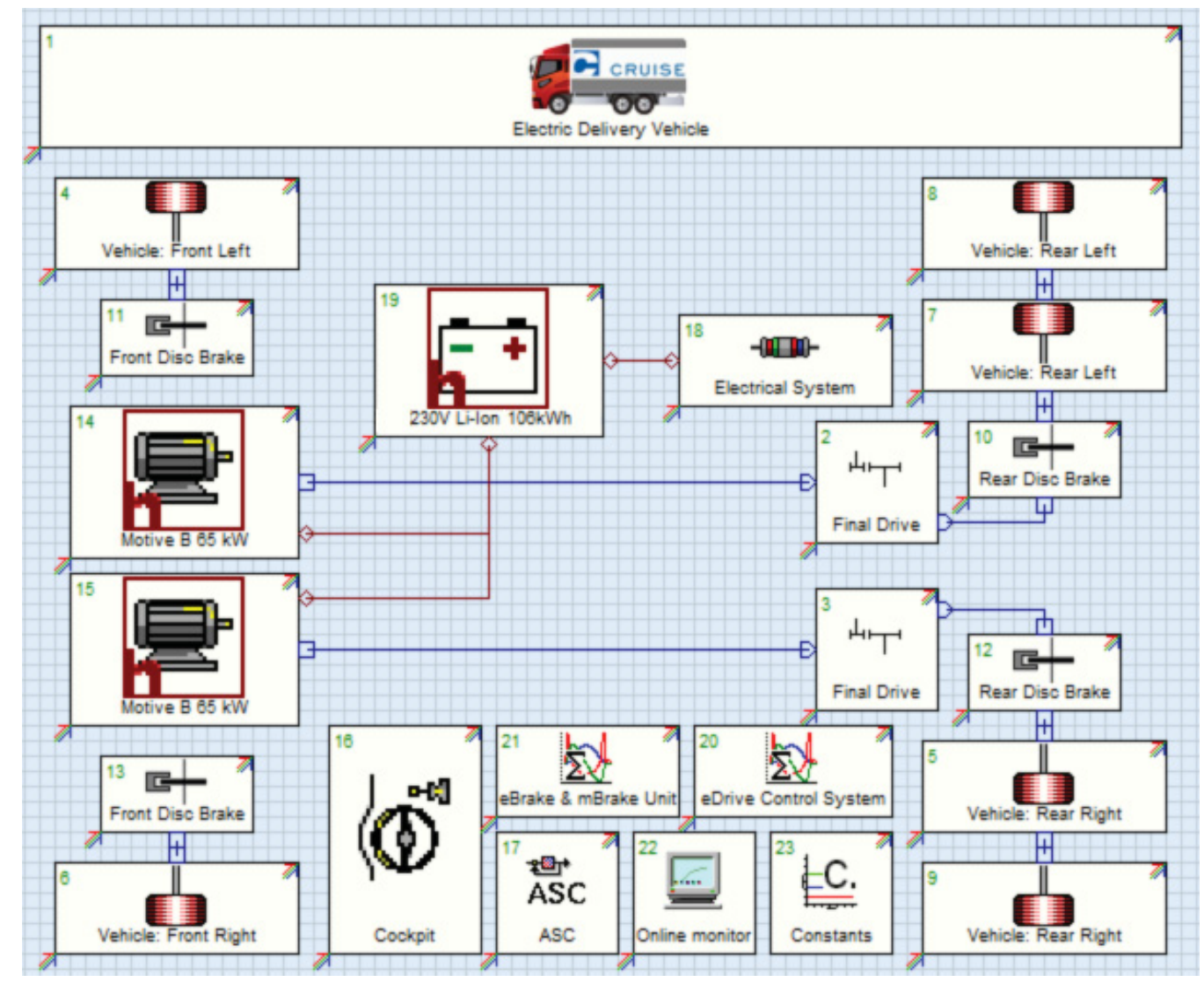

Figure 4-8: Vehicle Model in AVL Cruise

\subsubsection{New system, S2}

\subsubsection{Overall System design}

The differences between S1 and S2 are that the battery voltage is $230 \mathrm{~V}$ instead of $460 \mathrm{~V}$, and that the battery is connected to the inverters via a DC-DC converter. The total energy of the battery pack and the other vehicle parameters are identical to the baseline system. 


\subsubsection{DC-DC converter design}

The DC-DC converter was designed for an input voltage of $200 \mathrm{~V}$ and a maximum output voltage of $600 \mathrm{~V}$ for a conservative design. In order to obtain most advantage from S2 and expand the region of operation at high MIs, the system should operate at as low a battery voltage as possible. However, a low battery voltage requires that the DC-DC converter be operated at a high duty-cycle, which has two associated problems:

1. Larger passive components (inductors and capacitors) will be needed $[36,37]$.

2. The boosting capability of boost converters are lower at higher duty cycles due to parasitic elements [9].

By considering the minimum input voltage of $200 \mathrm{~V}$ and the maximum output voltage of $600 \mathrm{~V}$, the maximum duty cycle will be $66.67 \%$, which is in a medium range. This design is conservative enough to be used for all phases of the project. 


\subsubsection{Control Strategy}

The overall control algorithm for the variable DC bus voltage control is shown in Figure 4-9.

Figure 4-9 demonstrates that the duty cycle of the DC-DC converter is determined by its own control loop. In some publications the output current of the DC-DC converter is not considered in the control loop and only the output voltage of the DC-DC converter is considered. However, further examination showed that the dynamics of the rest of the system have a huge effect on this current or more specifically current disturbance. This current disturbance can result in drainage of the DC-link capacitor or conversely cause an over voltage. Therefore, the current had to be considered in the control loop. Furthermore, we can see that the control loop starts with the target modulation index. As was previously mentioned, this value varies during Phase 3 and 4 of the project and different values will be examined. Therefore, the control loop had to be redesigned multiple times and could not be

completed for this project. In order to simplify the system, it was assumed that the DC-link capacitor was large enough to keep the voltage completely constant in the steady state operation. This assumption allowed the decoupling of the system. As long as the DC-link voltage and current consumed by the load were known at any operating point, the two systems could be examined individually. Figure 4-10 demonstrates the decoupled system. 


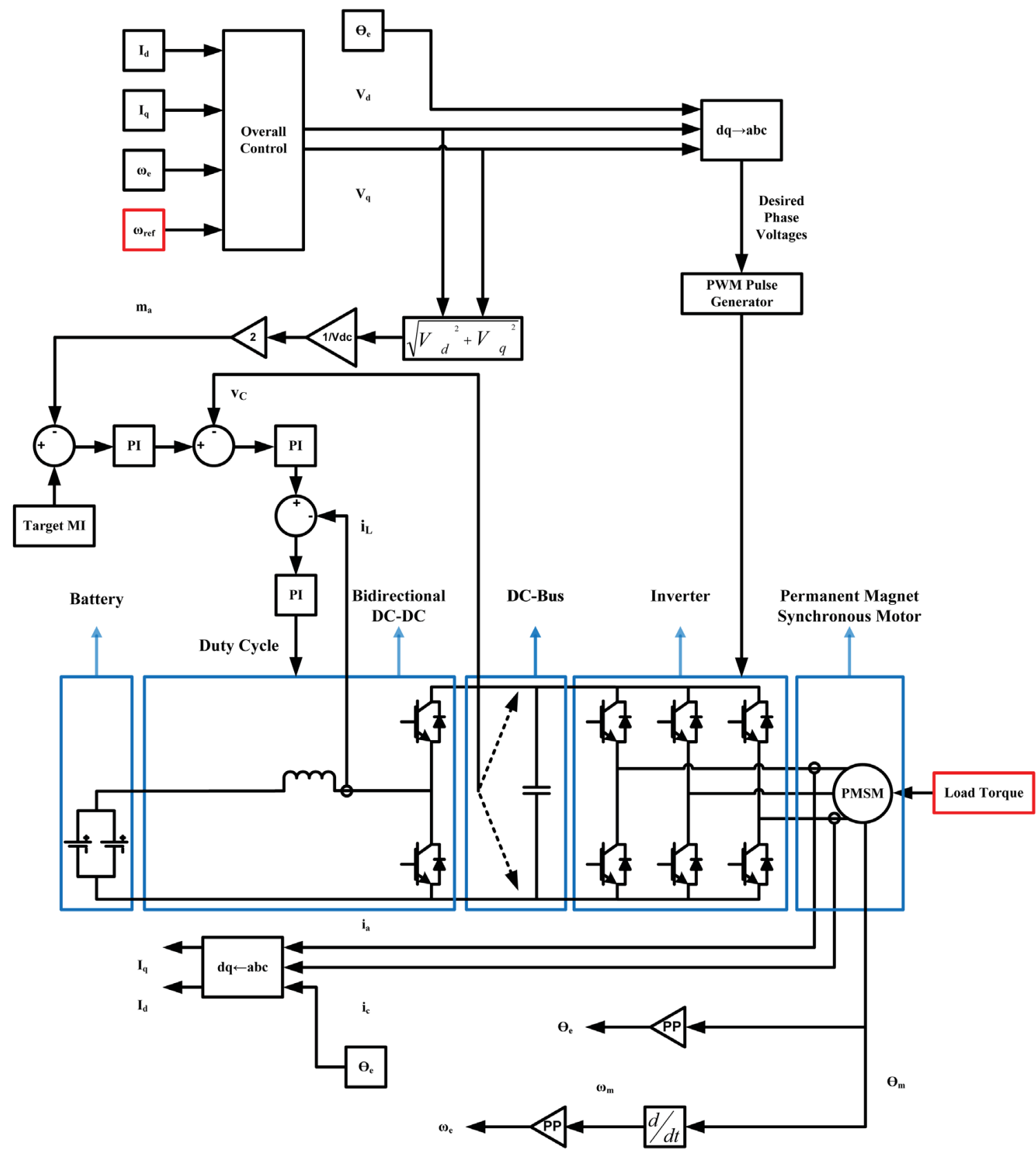

Figure 4-9: Overall control strategy for a variable DC bus voltage control 

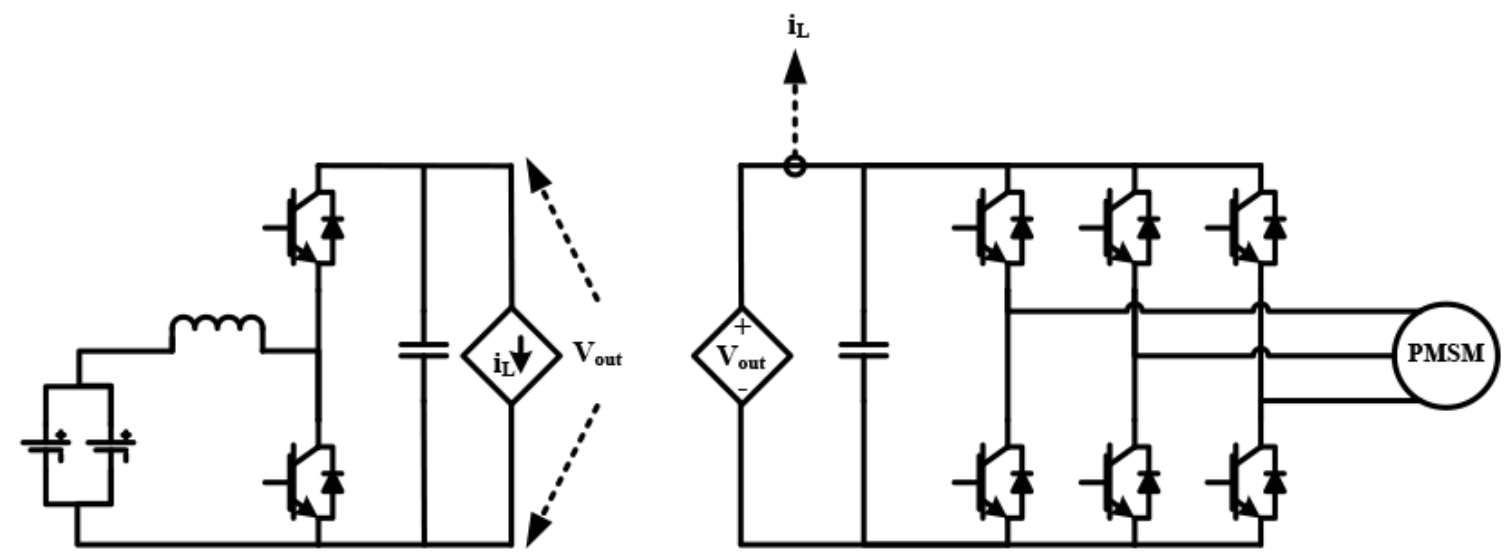

Figure 4-10: Decoupled System

If the DC-link voltage is known, this voltage can be applied to the inverter and the operation of the inverter and more specifically the DC current that it consumes can be determined. Therefore, the only parameter that needs to be established at any operation point is the DC link voltage. The control strategy of S2 allows the determination of this voltage. From Figure 4-11 several important points can be observed.

1. In Zone 1, the DC-DC converter will maintain the DC bus voltage at the battery level since the boost converter is not capable of lowering the voltage below its input voltage.

2. In Zone 3, the DC-DC converter will have to boost the DC bus voltage to the rated value of 460 Volts.

3. The only region where the DC-link voltage is unknown is Zone 2. However, in this region the MI must be equal to the target value. 


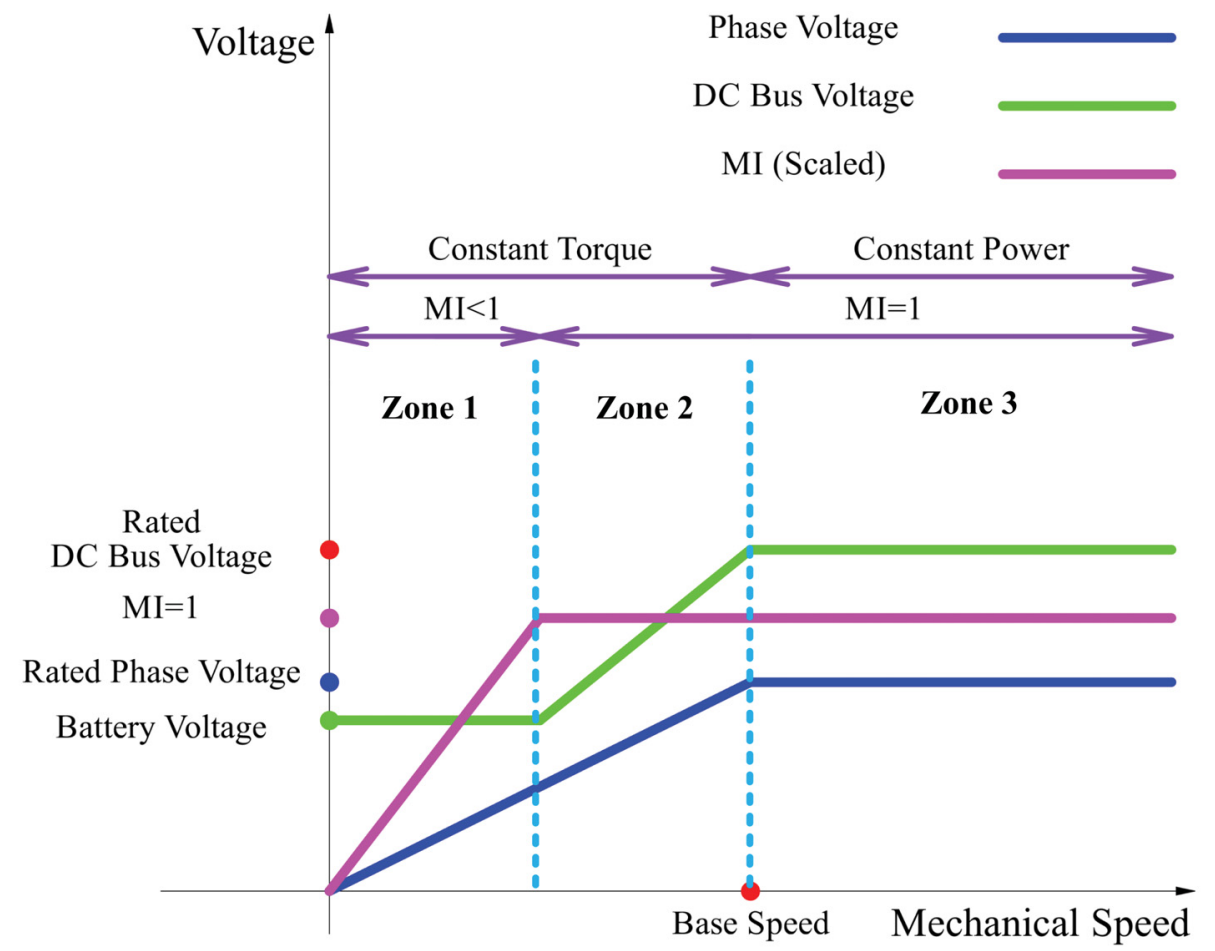

Figure 4-11: Zones of operation for S2

Therefore if the system is forced to operate at the target MI in Zone 2, the DClink voltage can be established. Based on the information gathered from the baseline system S1, a linear interpolation was used to estimate the DC-link voltage that was required to maintain the target $\mathrm{MI}$ in Zone 2. Afterwards, using the estimated value as a starting point and an optimization program, the DC-link voltage was tweaked until the MI matched the target MI. An error of less than $1 \%$ was permitted in order to limit the optimization time to a reasonable value. In order to determine the current pulled from the DC-DC converter $\left(I_{o}\right)$, the input power of the inverter $\left(P_{I N V_{-} I N}\right)$, the input power of the motor $\left(P_{M O T_{-} I N}\right)$, the inverter losses $\left(P_{I N V_{-} L O S S E S}\right)$, and the 
DC link voltage $\left(V_{d c}\right)$ were used. The following formulas were employed to do this.

$$
\begin{gathered}
P_{I N V_{-} I N}=P_{M O T}+P_{I N V_{-L O S S E S}} \\
I_{o}=\frac{P_{I N V_{-} I N}}{V_{d c}}
\end{gathered}
$$

The load seen by the DC-DC converter $\left(R_{o}\right)$ was determined to be:

$$
R_{o}=\frac{V_{d c}}{I_{o}}
$$

The losses of the DC-DC converter included the switching and conduction losses of the switches and diodes and the ohmic losses of the inductor due to its internal resistance. The overall control of the DC-DC converter was implemented in Simulink (Figure 4-12) and the DC-DC converter model was created in PSIM (Figure 4-13). In this part of the simulation, the DC-DC converter applies the desired DC bus voltage $\left(V_{d c}\right)$ to the equivalent load of the rest of the system $\left(R_{o}\right)$. Figure 4-14 shows a sample waveform of the output voltage and output current of the DC-DC converter and Figure 4-15 shows the DC-DC converter losses. The DC-DC converter efficiency $(D E)$ is calculated as:

$$
D E=\frac{P_{I N V_{-} I N}}{P_{I N V_{I} I N}+D C-D C_{L O S S E S}}
$$

Where, the $D C-D C_{L O S S E S}$ is the DC-DC converter losses. The overall system efficiency is determined as:

$$
S E=M E \times I E \times D E
$$




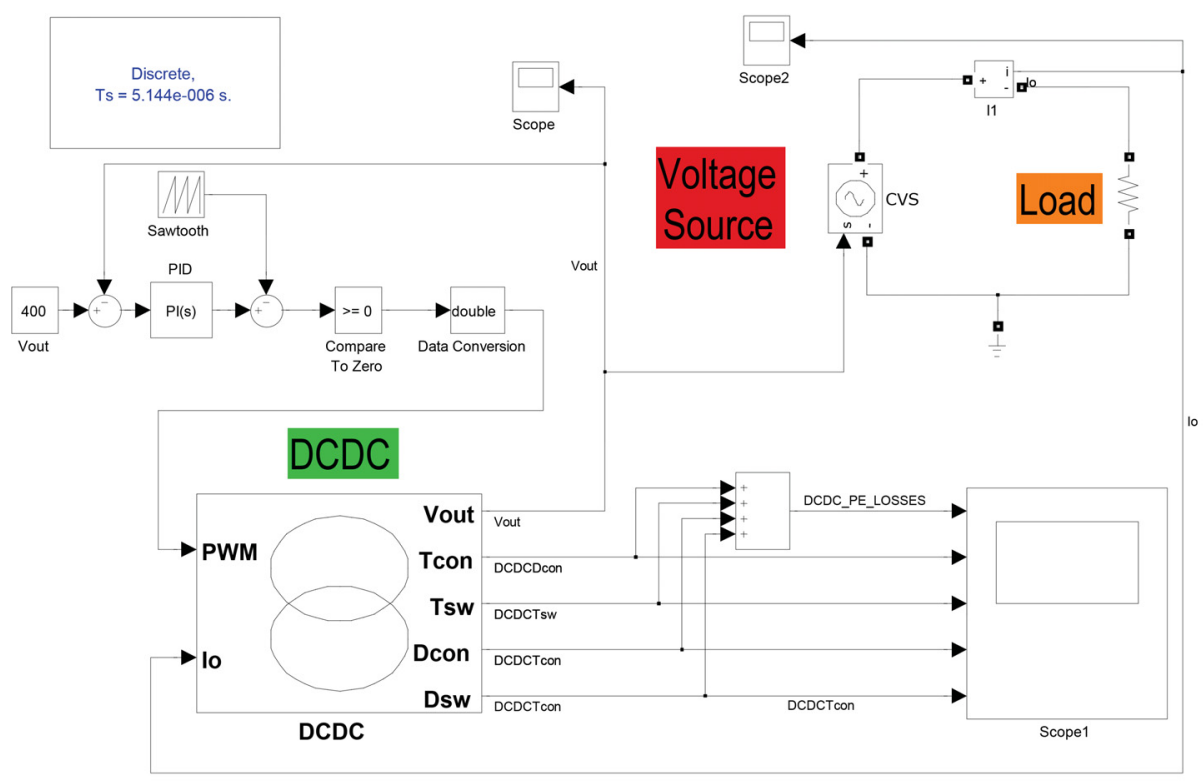

Figure 4-12: Control of the DC-DC converter in Matlab

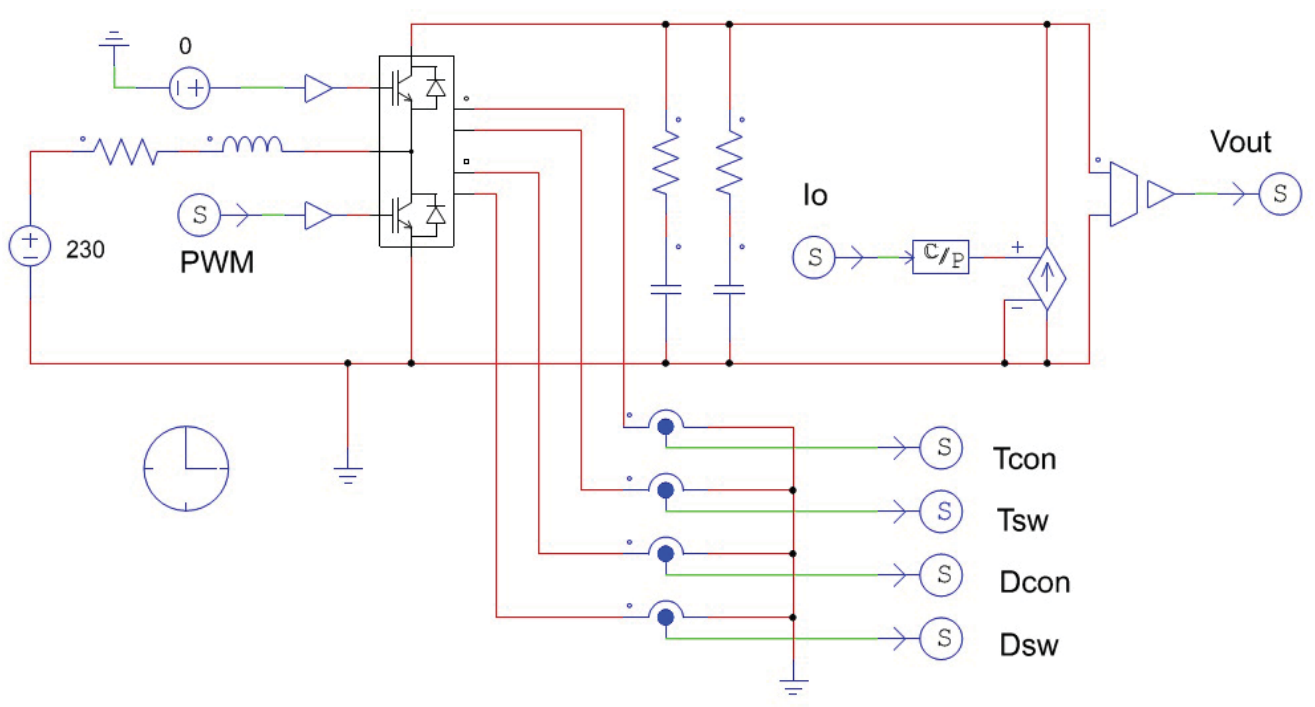

Figure 4-13: Implementation of the DC-DC converter in PSIM 

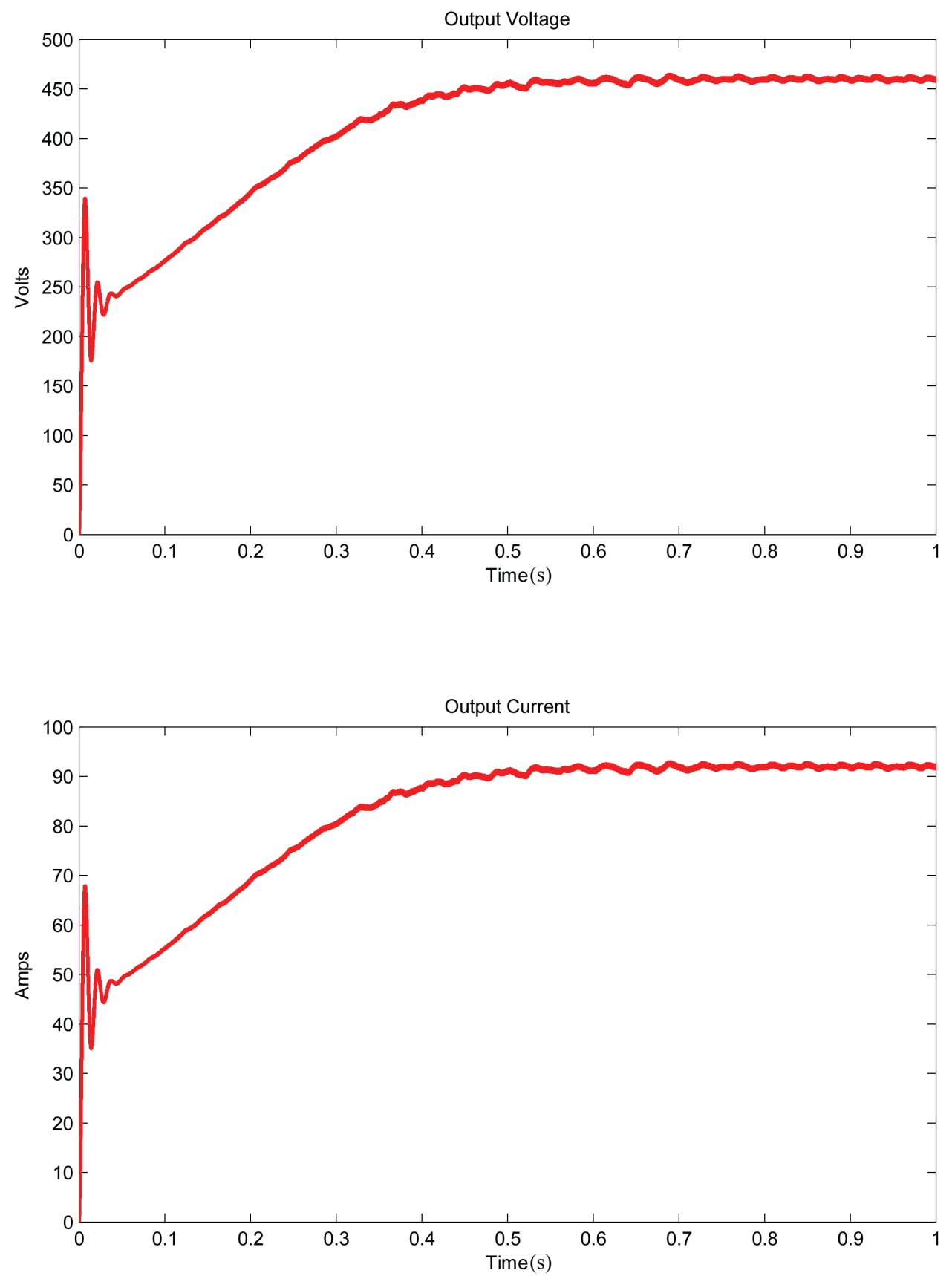

Figure 4-14: Sample operation of the DC-DC converter 

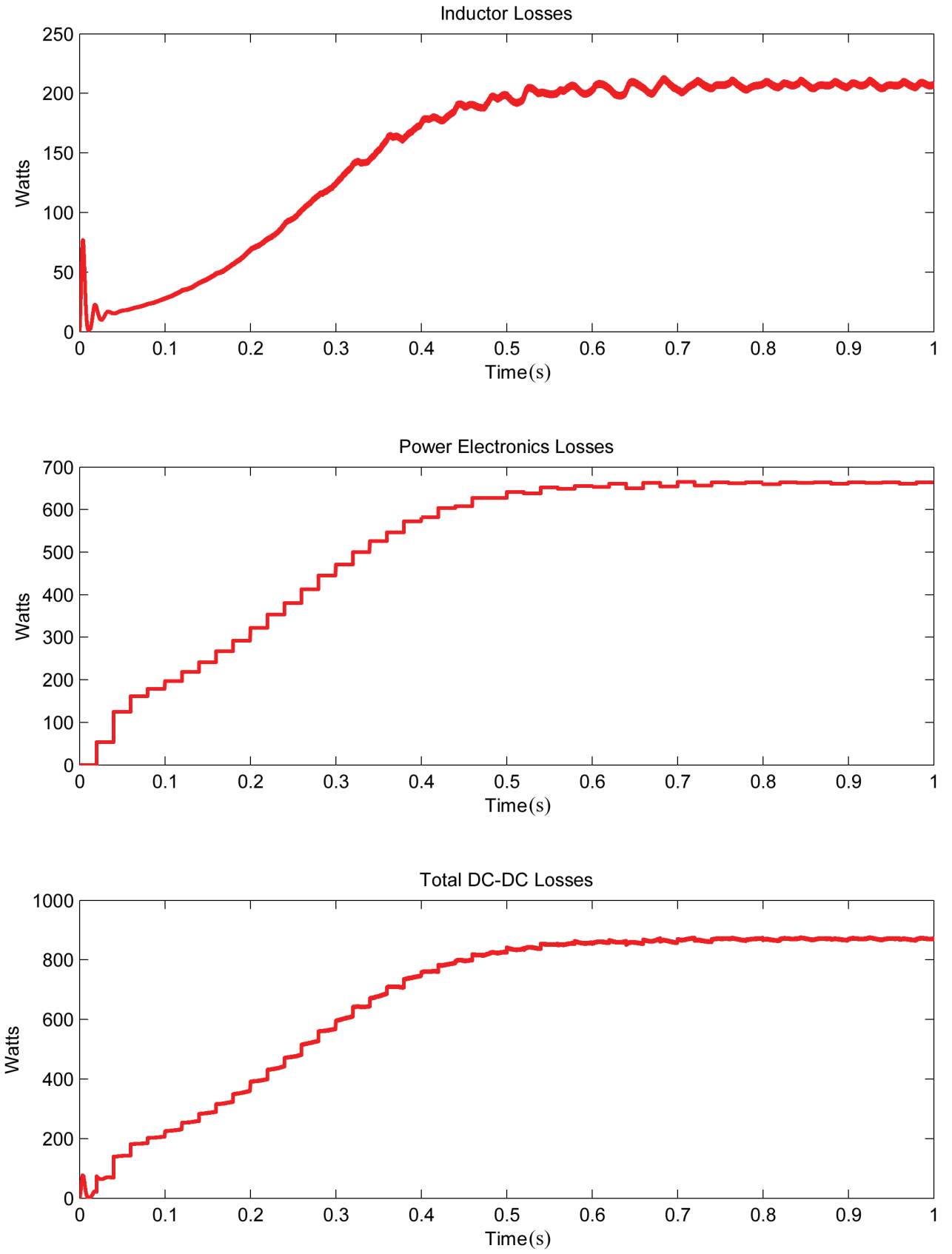

Figure 4-15: DC-DC converter Losses 
The total steady state losses of the DC-DC converter shown in Figure 4-15 are $870.2 \mathrm{~W}$ on average, which may seem high. However the input power for this particular operation point is $42.73 \mathrm{~kW}$, which brings the DC-DC converter efficiency to $98 \%$.

It is important to remember that since the system has been simplified, it produces a more conservative result for efficiency and THD compared to a more sophisticated model. This means that the efficiencies are higher and the THDs are lower compared to the results produced by a more sophisticated model.

For this phase of the project the two systems are compared to each other by running a simulation for each over the Orange County Cycle (Figure 4-16). This cycle approximates the routes taken by medium duty delivery trucks very well $[11,33]$. It can be seen that this cycle contains low speeds and lots of start and stop cycles.

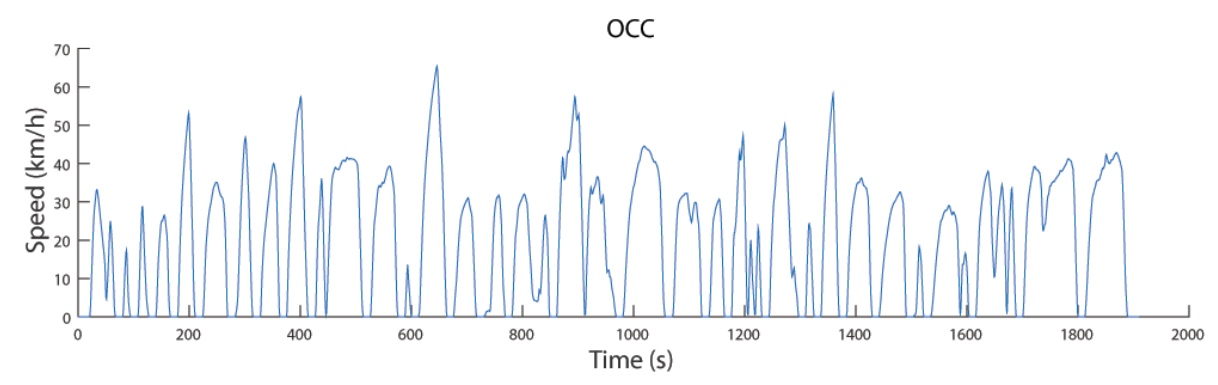

Figure 4-16: Orange County Cycle

\subsection{Phase 2}

The same efficiency maps as in phase 1 are used for this section. The main objective of this section is to examine the effectiveness of the new system in terms of different drive cycles. 5 standard drive cycles (Figures 4-17) applicable to delivery trucks and 5 actual drive cycles (Figures 4-18) are used for this section. Among the 
standard drive cycles, the OCC was already discussed. The NYCC and the HTUF4 cycles are also applicable to medium duty delivery trucks and have low speeds and lots of start and stop cycles [33]. The UDDS cycle represents the urban cycle and demonstrates the behavior of the vehicle in the city [1]. Similar to the other cycles, the UDDS has low average speeds and lots of start and stop cycles. The UDDSHD cycle [34] does not represent the behavior of a delivery truck since it has a higher average speed and some constant speed operation. This cycle is used for comparison of the results with the other cycles.

The 5 actual drive cycles are based on several days of testing that were conducted with delivery vehicles in use on real delivery routes. The captured data allowed the creation of speed/time drive cycles to be used in simulation. The cycles all began and ended at one of three delivery hubs located in the Montreal area: Anjou, Lachine, and Laval. Anjou is a suburban area to the northeast of downtown Montreal and these routes include small highway sections as well. Lachine is in the southwest of Montreal, but the routes from this hub take the trucks directly into the downtown core. The final route location is Laval, which is north of Montreal and includes mostly suburban and highway sections. On all routes, the trucks are supposed to be shut down during delivery stops. This eliminates accessory load drains during stops. Any stops longer than five minutes were deemed delivery stops in all cycles and the simulation truck accessory loads were set to zero for these periods of time.

The average speed and the kinetic intensity of the cycles are used to characterize theses drive cycles and identify them as suitable cycles for S2. 


\subsection{Phase 3}

As mentioned in Section 3.2.3, the target MI can be changed in phase 3 of the project. In this section, the same methodology as Phase 1 is employed. The only difference is the fact that the MI is changed and the effectiveness of the system is examined for different target MIs during the same 10 cycles.

\subsection{Phase 4}

In this phase, three different battery packs at voltages of $200 \mathrm{~V}, 230 \mathrm{~V}$, and 300 $\mathrm{V}$ are designed. The energy capacity of these packs is kept roughly at the same level. Once again the same methodology as phase 1 is applied to determine the efficiency maps for the different systems at different MIs. The effectiveness of the systems are evaluated over the same 10 drive cycles. 

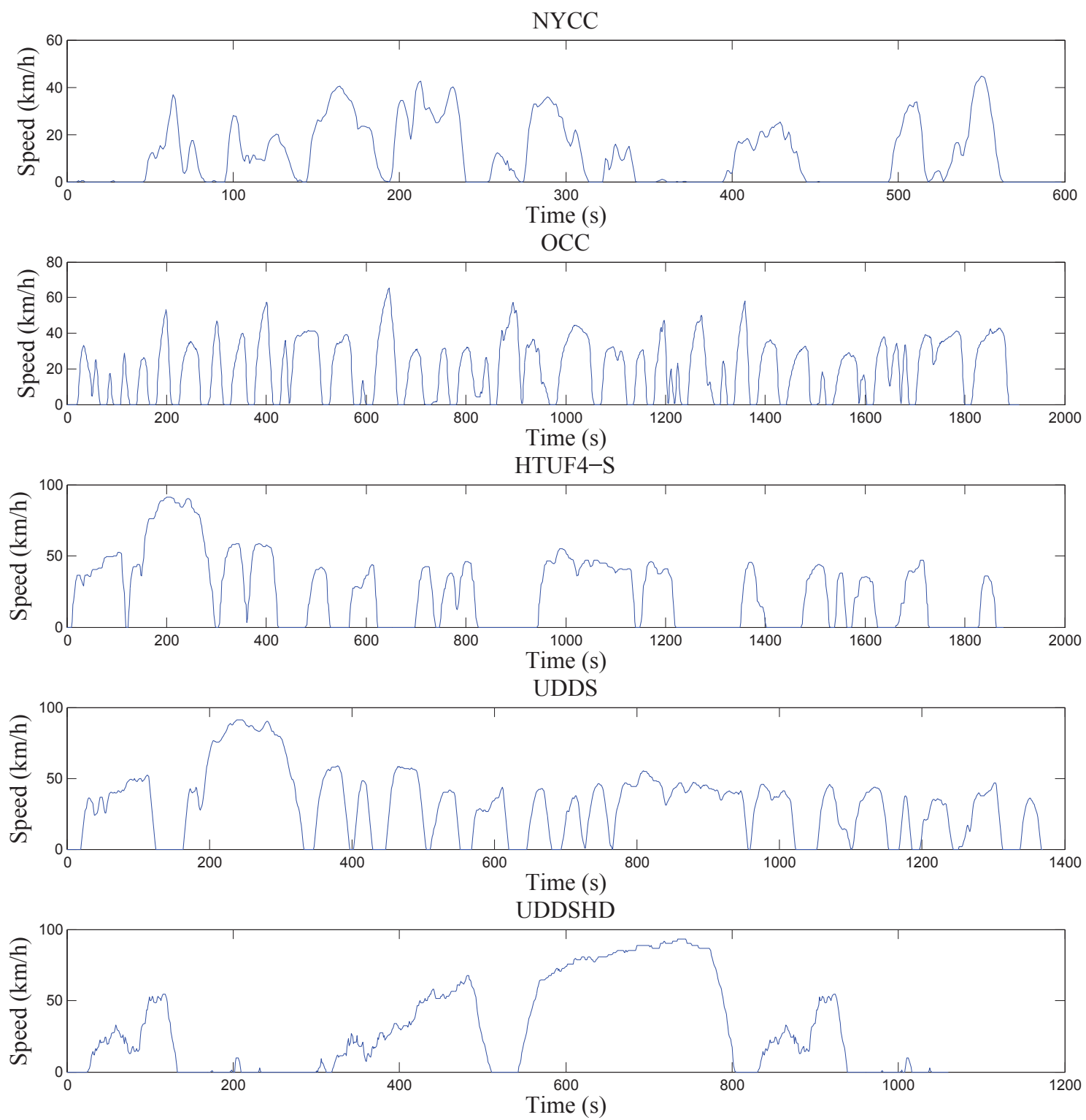

Figure 4-17: Speed traces of standard drive cycles: NYCC, OCC, HTUF4-S, UDDS, and UDDSHD. 
Anjou 1

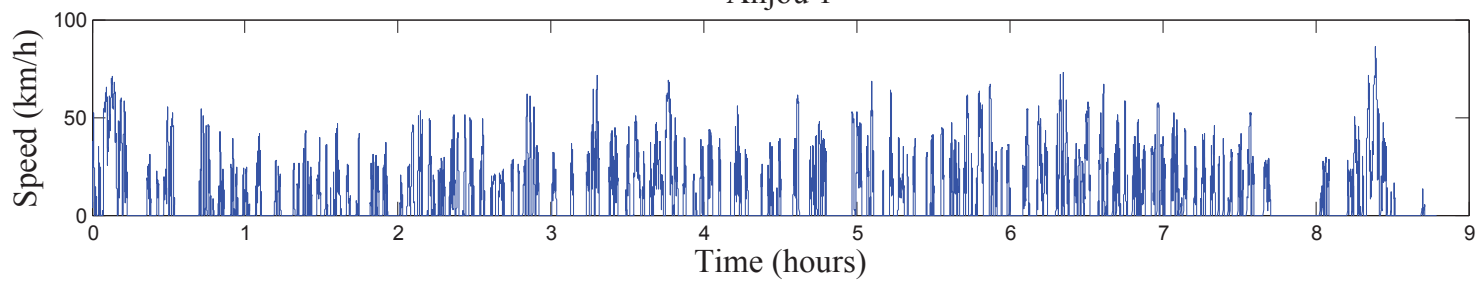

Anjou 2

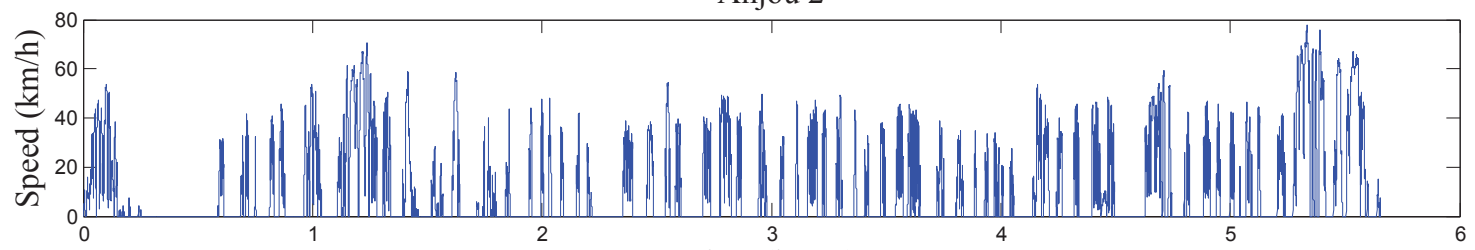

Time (hours)
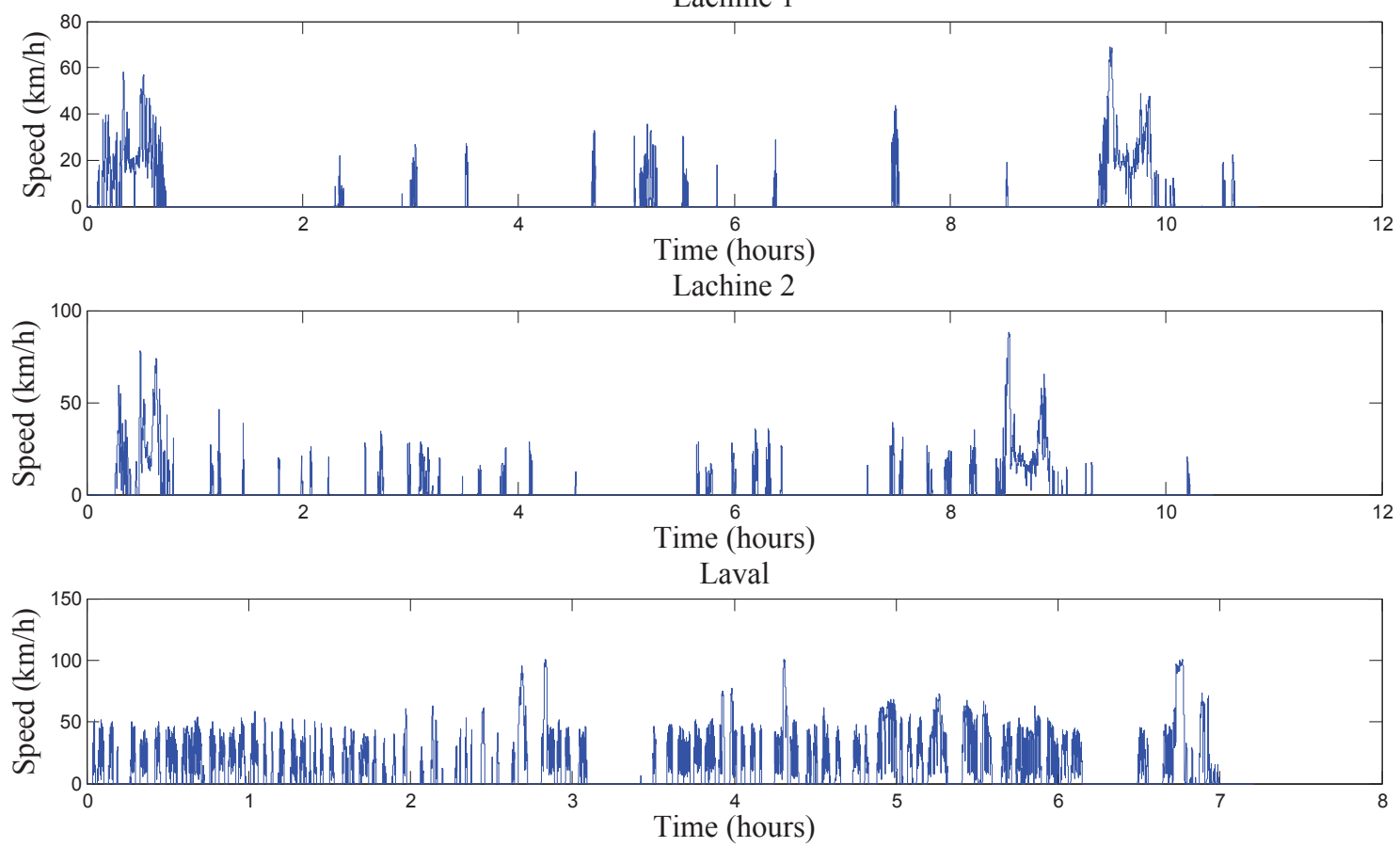

Figure 4-18: Speed traces of recorded daily drive cycles for delivery trucks in Anjou, Lachine, and Laval. 


\section{Chapter 5 \\ Results}

\subsection{Phase 1}

Figures 5-1 and 5-2 show the efficiency maps that were obtained for S1 and S2. The inverter and motor efficiency have increased in the low RPM region. The same behavior is visible for the global efficiency, however the high RPM operation regions of the global efficiency show a reduction due to the high DC-DC converter losses in this region.

An easy way to visualize the difference in the efficiency between the two systems is to look at their Percent Efficiency Difference (PED) for all the operation points. This value is calculated as the difference in efficiency of the system normalized by the efficiency of the base model. The following equation describes this value.

$$
P E D=100 \times \frac{E S 2-E S 1}{E S 1}
$$

In this equation, ES1 and ES2 are respectively the global efficiency of S1 and S2 for a given point. Therefore, a positive PED will represent an improvement in efficiency and a negative PED will represent a degradation in efficiency introduced by S2. Figure 5-3 shows the PED map for all the operation points in the torque-speed envelope. In this figure it can clearly be observed that S2 has a higher efficiency in 
the low RPM region and lower efficiency in the high operation region compared to S1.

Similarly, Percent THD Difference (PTD) can be defined to compare the changes in THD. The following equation describes this value.

$$
P T D=100 \times \frac{T H D S 2-T H D S 1}{T H D S 1}
$$

In this equation, THDS1 and THDS2 are respectively are the THD of S1 and S2 for a given point. Therefore, a positive PTD will represent an improvement in THD and a negative PTD will represent a degradation in THD introduced by S2. Figure 5-4 shows the PTD map for all the operation points in the torque-speed envelope. In this figure it can clearly be seen that S2 has a lower THD in the low RPM region and a higher THD in the high operation region compared to S1.

The energy consumption (EC) was calculated by dividing the energy use by the cycle distance and allows a reasonable comparison between the cycles for the future phases.

$$
\text { EnergyConsumption }(W h / k m)=\frac{\operatorname{EnergyU} \operatorname{se}(W h)}{\text { CycleDistance }(k m)}
$$

The percentage improvement was calculated using the energy consumption for the S1 system, compared with the energy consumption of the S2 system as shown in the following equation.

$$
\text { PercentImprovement }=100 \times \frac{E \operatorname{Cof} S 1-E \operatorname{Cof} S 2}{E \operatorname{Cof} S 1}
$$


The results are consistent with the literature review and the improvement introduced by S2 in terms of efficiency and THD. Based on the simulations produced by AVL Cruise, S1 uses $763 \mathrm{Wh} / \mathrm{km}$ and S2 uses $748 \mathrm{Wh} / \mathrm{km}$ through the OCC cycle. Figure 5-5 shows the operation points of each motor during the OCC cycle, as produced by AVL Cruise. The figure clearly shows that the majority of the operation points are in the low RPM region. This explains why S2 improved the energy consumption of the system through OCC cycle. This energy saving equates to an improvement of $1.6 \%$ during the OCC. 

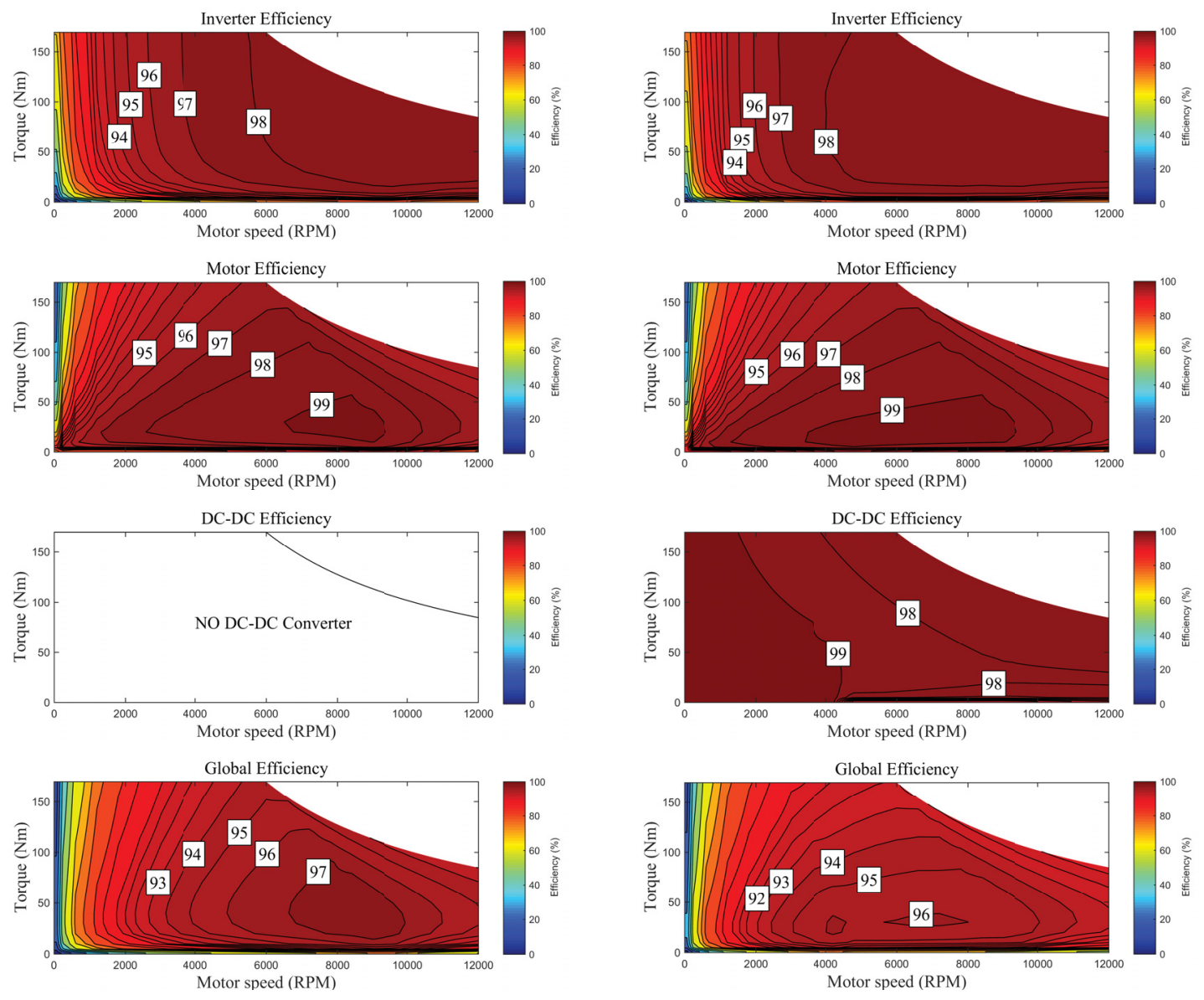

Figure 5-1: Efficiency maps for S1

Figure 5-2: Efficiency maps for S2 


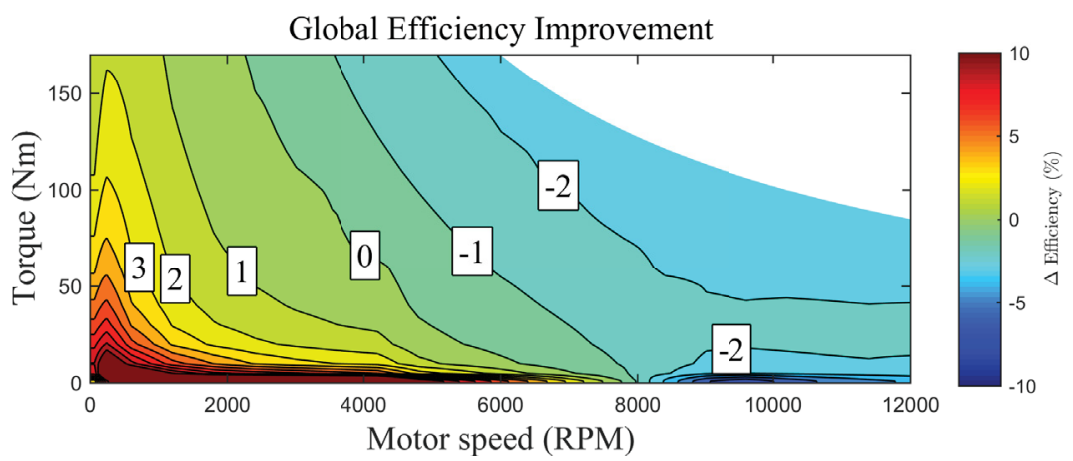

Figure 5-3: Efficiency difference between S1 and S2

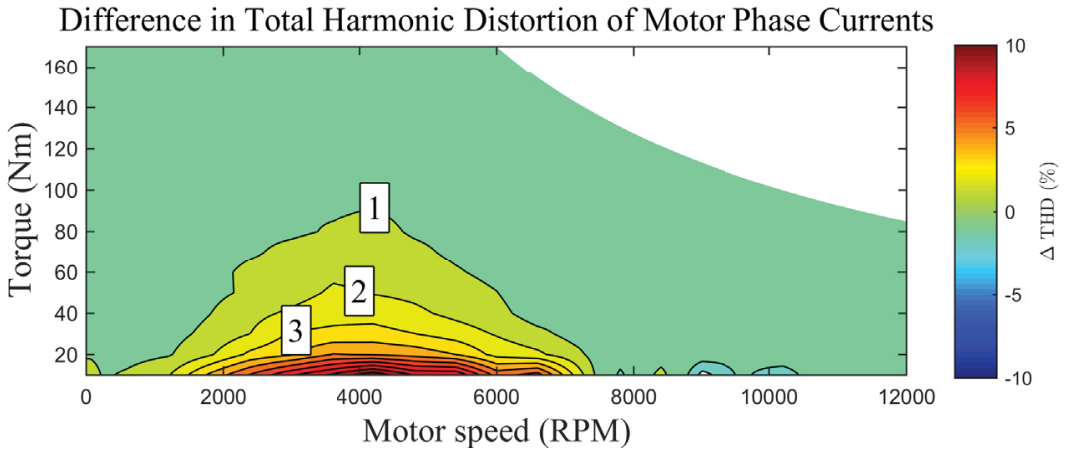

Figure 5-4: Difference in THD between S1 and S2

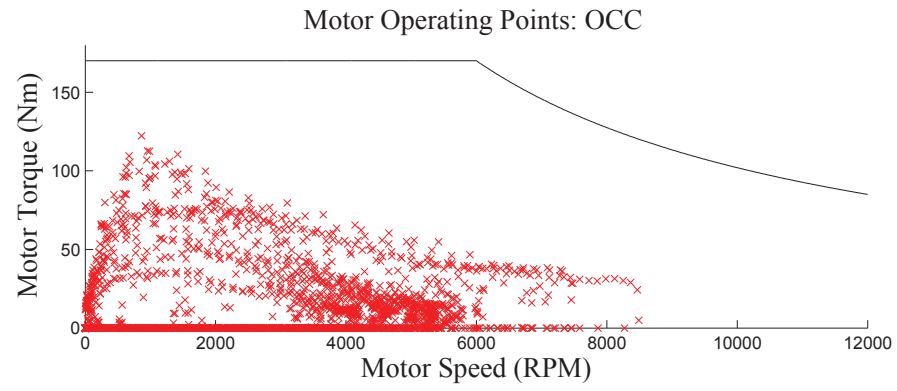

Figure 5-5: Operating point maps for OCC 


\subsection{Phase 2}

In this phase of the project, the effectiveness of $\mathrm{S} 2$ is studied in terms of multiple drive cycles. Kinetic intensity and average driving speed of these cycles are used for characterization purposes.

The results for some cycles are of particular interest. S2 introduced a $2 \%$ energy savings for NYCC which was the largest improvement among the standard cycles. This is not surprising since this cycle has the highest kinetic intensity and the lowest average speed. Furthermore, if we look at the operation points of this cycle, it can be seen that all the operations points are in the low RPM region.

S2 increased the energy usage for UDDSHD by $0.8 \%$. This is due to the fact that this cycle has the lowest kinetic intensity and the highest average speed among all the cycles. Looking at the operation points of the motor for this cycle, there are many high speed operation points. It was mentioned earlier that this cycle is used for comparison purposes and it does not represent the usual behavior of a delivery truck.

From the results of all the cycles, it is clear that S2 could be beneficial for cycles with high kinetic intensity and low average speeds. The best result obtained from $\mathrm{S} 2$ was during the Lachine 1 cycle which resulted in $2.2 \%$ in energy savings. 

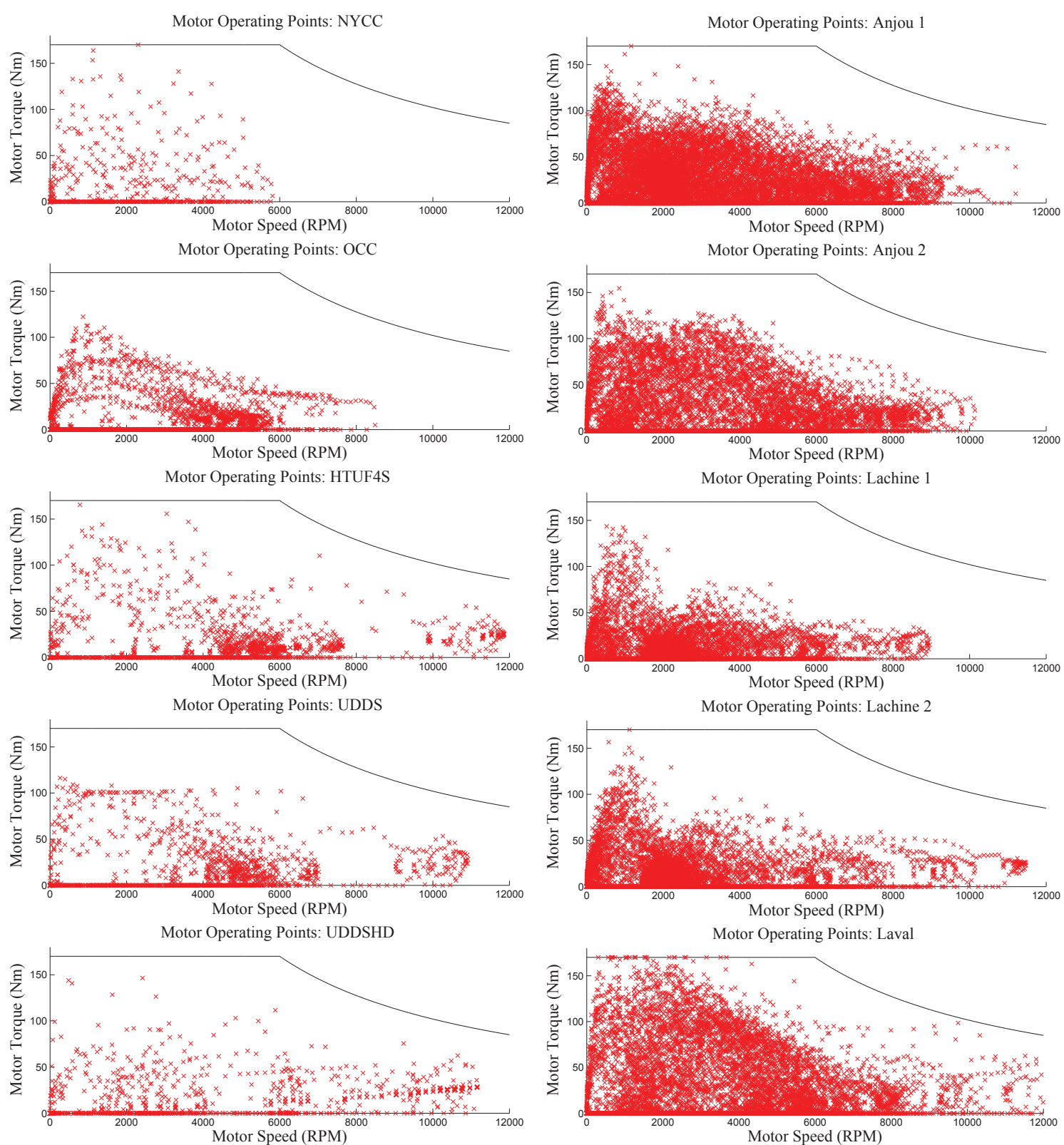

Figure 5-6: Operating point maps for all cycles, standard cycles on the left and road tests on the right. 
Table 5-1: Drive cycle metrics and vehicle simulation results for standard and recorded drive cycles for Phase 2

\begin{tabular}{|c|c|c|c|c|c|c|c|}
\hline & \multicolumn{2}{|c|}{$\begin{array}{l}\text { DRIVE CYCLE } \\
\text { METRICS }\end{array}$} & \multicolumn{2}{|c|}{ S1 RESULTS } & \multicolumn{2}{|c|}{ S2 RESULTS } & \multirow[b]{2}{*}{$\begin{array}{l}\text { Diff } \\
\%\end{array}$} \\
\hline & $\begin{array}{l}\text { Kinetic } \\
\text { Int. } \\
\mathrm{km}^{-1}\end{array}$ & $\begin{array}{l}\text { Avg } \\
\text { Speed } \\
\mathrm{km} / \mathrm{h}\end{array}$ & $\begin{array}{l}\text { Energy } \\
\text { Used } \\
\text { kWh }\end{array}$ & $\begin{array}{l}\text { Energy } \\
\text { Cons. } \\
\text { Wh/km }\end{array}$ & $\begin{array}{l}\text { Energy } \\
\text { Used } \\
\text { kWh }\end{array}$ & $\begin{array}{l}\text { Energy } \\
\text { Cons. } \\
\text { Wh/km }\end{array}$ & \\
\hline NYCC & 5.137 & 16.8 & 1.5 & 763 & 1.4 & 748 & 2.0 \\
\hline $\mathrm{OCC}$ & 2.231 & 24.7 & 6.3 & 595 & 6.2 & 585 & 1.6 \\
\hline HTUF4S & 0.622 & 38.8 & 7.1 & 542 & 7.1 & 541 & 0.2 \\
\hline UDDS & 0.798 & 38.9 & 6.9 & 573 & 6.9 & 573 & 0.0 \\
\hline UDDSHD & 0.378 & 45.4 & 5.7 & 641 & 5.8 & 646 & -0.8 \\
\hline Anjou1 & 2.017 & 23.8 & 60.1 & 678 & 59.4 & 670 & 1.2 \\
\hline Anjou2 & 2.119 & 26.0 & 43.8 & 751 & 43.5 & 745 & 0.7 \\
\hline Lachine1 & 1.634 & 20.0 & 22.1 & 715 & 21.7 & 700 & 2.2 \\
\hline Lachine2 & 1.352 & 19.7 & 25.3 & 726 & 24.8 & 713 & 1.9 \\
\hline Laval & 1.783 & 31.2 & 86.4 & 817 & 86.4 & 817 & 0.0 \\
\hline
\end{tabular}

\subsection{Phase 3}

The objective of this section was to discover if there is anything else that can be done in order to increase the effectiveness of S2. Figure 5-7 shows some examples of PED maps compared to S1 for various target MIs. S2 has increased the system efficiency in the low RPM region for all target MIs. However, the degree of improvement varies significantly as the target MI changes. It can be seen that the zero contour which essentially represents the borderline between improvement and degradation moves to the right as the MI is increased. Furthermore, in the 
high RPM region, the overall efficiency decreased significantly. The efficiency maps differ significantly from each other and the effectiveness of each configuration must be examined in the context of an appropriate drive cycle.

Figure 5-8 shows some examples of PTD maps compared to S1 for various target MIs. The THD was improved in the low RPM region for all the MIs. When the MI increased above ' 1 ', in the high RPM range a significant improvement was observed. As previously mentioned, this is an overestimation of the improvement. The variation in DC bus voltage in a more sophisticated model would likely lower these values.

The AVL Cruise vehicle powertrain simulations show varying degrees of improvement for the different MIs. Figures 5-9 and 5-10 graphically represent the energy consumption of the S2 system at different values of MI for standard and captured cycles respectively. For several cycles including the HTUF4S, UDDS, UDDSHD, Lachine 2, and Laval cycles there is an obvious minimum shown in the energy consumption. This minimum shows the optimum MI to be used for that particular cycle. Other cycles show a flattening of energy consumption curve at higher MIs. 

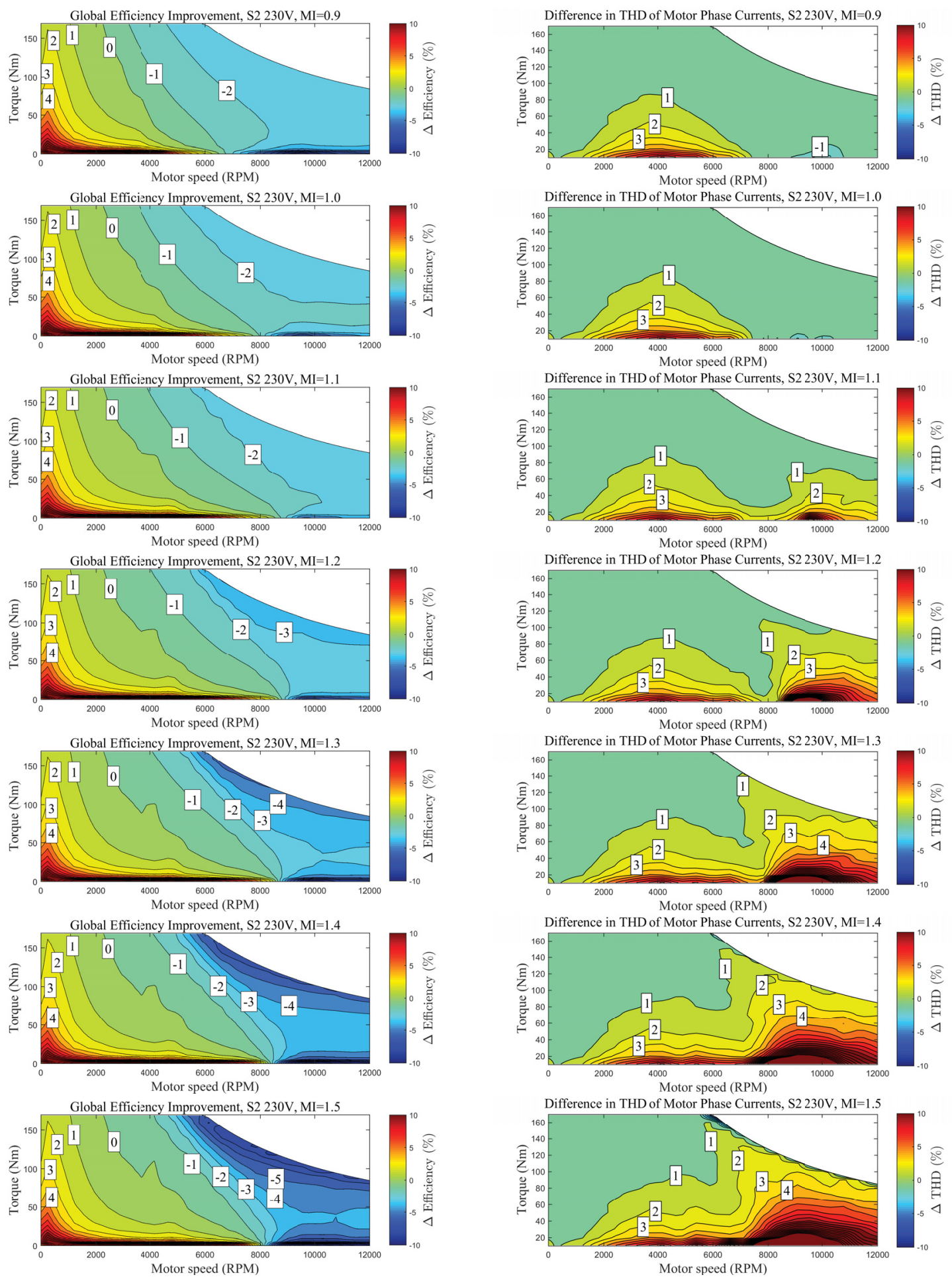

Figure 5-7: PED map for various MIs

Figure 5-8: PTD map for various MIs 

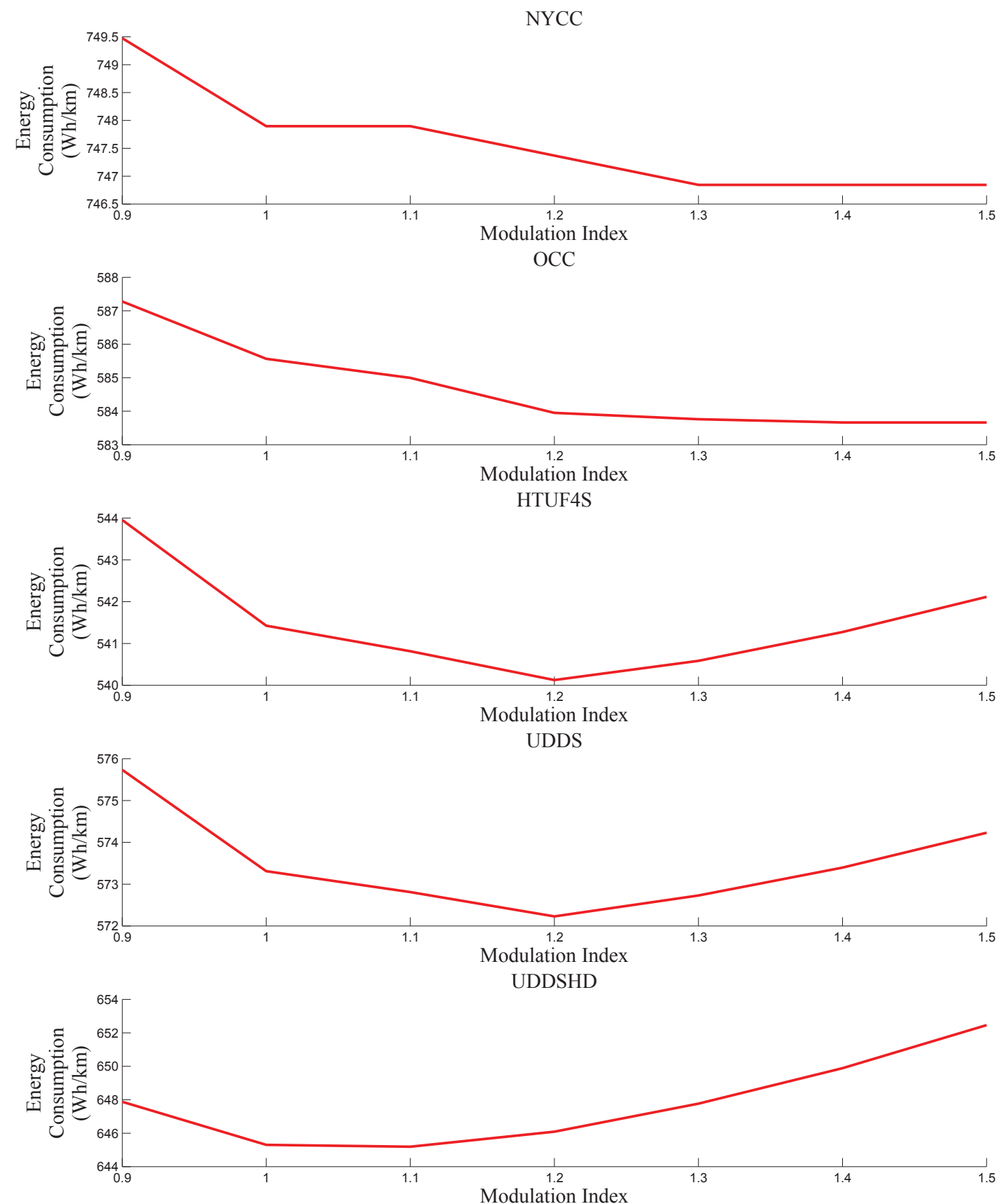

Figure 5-9: Energy consumption of S2 for various MI values during standard drive cycles 

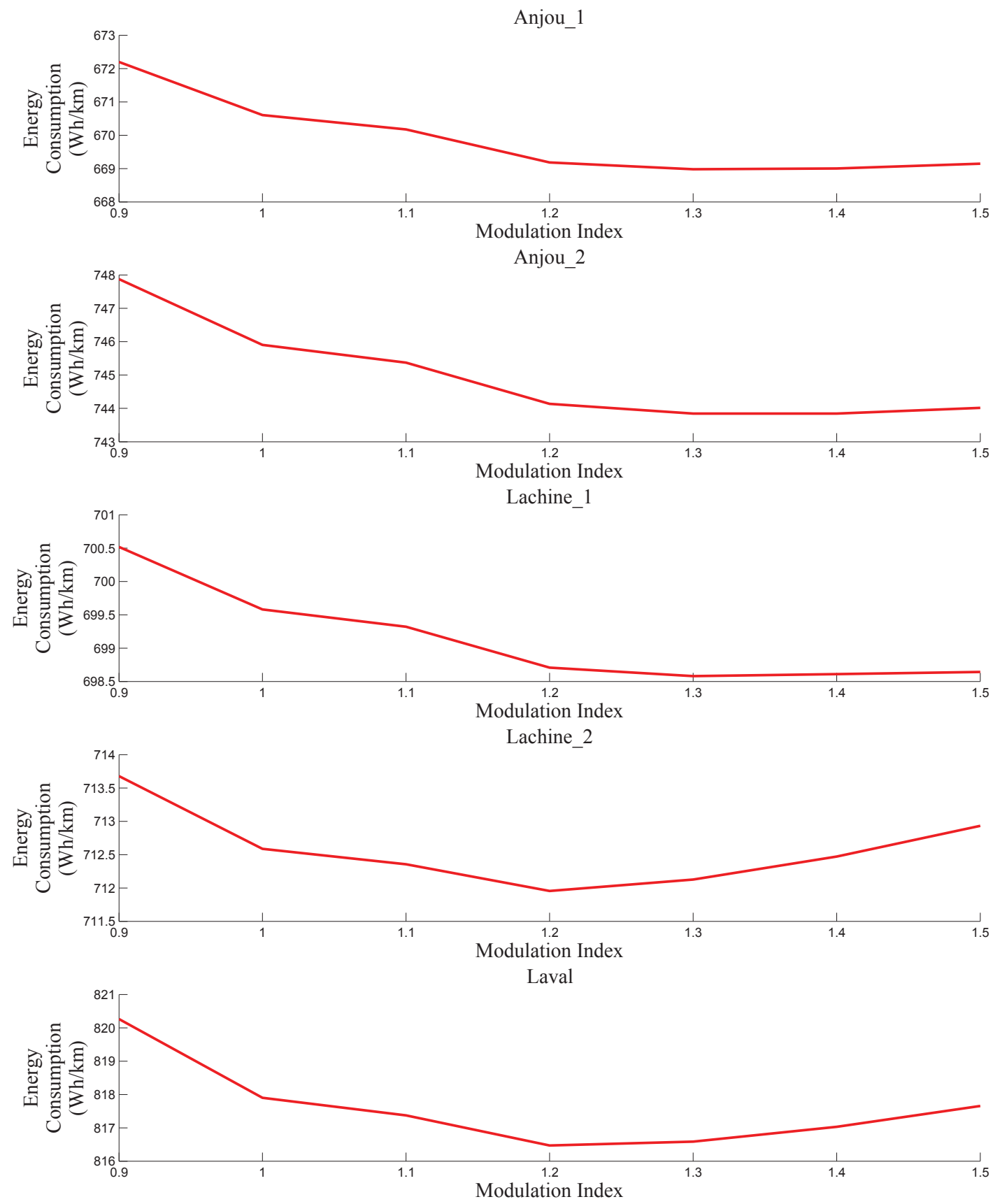

Figure 5-10: Energy consumption of S2 for various MI values during captured test cycles 
Table 5-2: Vehicle simulation results for Phase 3

\begin{tabular}{|c|c|c|c|c|c|c|}
\hline & \multicolumn{2}{|c|}{ S1 RESULTS } & \multicolumn{3}{|c|}{ S2 BEST RESULTS } & \multirow[b]{2}{*}{$\begin{array}{l}\text { Diff } \\
\%\end{array}$} \\
\hline & $\begin{array}{l}\text { Energy } \\
\text { Used } \\
\text { kWh }\end{array}$ & $\begin{array}{l}\text { Energy } \\
\text { Cons. } \\
\text { Wh/km }\end{array}$ & $\begin{array}{l}\text { Mod. } \\
\text { Index } \\
\text { - }\end{array}$ & $\begin{array}{l}\text { Energy } \\
\text { Used } \\
\text { kWh }\end{array}$ & $\begin{array}{c}\text { Energy } \\
\text { Cons. } \\
\text { Wh/km }\end{array}$ & \\
\hline NYCC & 1.45 & 763 & $1.3-1.5$ & 1.42 & 747 & 2.1 \\
\hline OCC & 6.27 & 595 & $1.4-1.5$ & 6.15 & 583 & 1.9 \\
\hline HTUF4S & 7.08 & 542 & 1.2 & 7.05 & 540 & 0.4 \\
\hline UDDS & 6.88 & 573 & 1.2 & 6.86 & 572 & 0.2 \\
\hline UDDSHD & 5.73 & 641 & 1.1 & 5.77 & 646 & -0.8 \\
\hline Anjou1 & 60.08 & 678 & 1.3 & 59.24 & 669 & 1.4 \\
\hline Anjou2 & 43.80 & 751 & $1.3-1.4$ & 43.37 & 743 & 1.0 \\
\hline Lachine1 & 22.14 & 715 & 1.3 & 21.63 & 699 & 2.3 \\
\hline Lachine2 & 25.27 & 726 & 1.2 & 24.78 & 712 & 1.9 \\
\hline Laval & 86.37 & 817 & 1.2 & 86.20 & 815 & 0.2 \\
\hline
\end{tabular}

The least percent improvement was $-0.8 \%$ for the UDDSHD cycle and the most improvement was $2.3 \%$ for the Lachine 1 cycle. Table 5-2 includes the energy use of S1 for each cycle and the best test for S2 for each cycle that was tested.

Table 5-3 summaries the drive cycle parameters and compares the improvement obtained from Phase 2 and Phase 3. In the majority of cases the strategy applied in Phase 3 has improved the energy consumption compared to Phase 2. The only two cases that did not improve by Phase 3 were the UDDSHD and Lachine 2 cycles. Once more it can be seen that drive cycles with high kinetic intensity and low average speeds benefit the most from S2. 
Table 5-3: Drive cycle metrics with Phases 2 and 3

\begin{tabular}{|c|c|c|c|c|}
\hline & \multicolumn{2}{|c|}{ METRICS } & \multirow{2}{*}{$\begin{array}{c}\text { Phase } 2 \\
\text { Diff } \\
\%\end{array}$} & \multirow{2}{*}{$\begin{array}{c}\text { Phase } 3 \\
\text { Diff } \\
\%\end{array}$} \\
\hline & $\begin{array}{c}\text { Kinetic } \\
\text { Int. } \\
\mathrm{km}^{-1}\end{array}$ & $\begin{array}{c}\text { Avg } \\
\text { Speed } \\
\mathrm{km} / \mathrm{h}\end{array}$ & & \\
\hline NYCC & 5.137 & 16.8 & 2.0 & 2.1 \\
\hline $\mathrm{OCC}$ & 2.231 & 24.7 & 1.6 & 1.9 \\
\hline HTUF4S & 0.622 & 38.8 & 0.2 & 0.4 \\
\hline UDDS & 0.798 & 38.9 & 0.0 & 0.2 \\
\hline UDDSHD & 0.378 & 45.4 & -0.8 & -0.8 \\
\hline Anjou1 & 2.017 & 23.8 & 1.2 & 1.4 \\
\hline Anjou2 & 2.119 & 26.0 & 0.7 & 1.0 \\
\hline Lachine1 & 1.634 & 20.0 & 2.2 & 2.3 \\
\hline Lachine2 & 1.352 & 19.7 & 1.9 & 1.9 \\
\hline Laval & 1.783 & 31.2 & 0.0 & 0.2 \\
\hline
\end{tabular}

\subsection{Phase 4}

Since the optimization of Phase 3 still only slightly improved the performance of the system, the effect of the battery voltage was also studied. Figures 5-11 and 5-12 show PEDs for S2 at various MIs and voltages.

The areas of improvement of S2 for different MIs and battery voltages vary quite a bit and therefore they must be studied in the context of the drive cycles. Figures 5-13 and 5-14 show the energy consumption of the system for different MIs and voltages. 

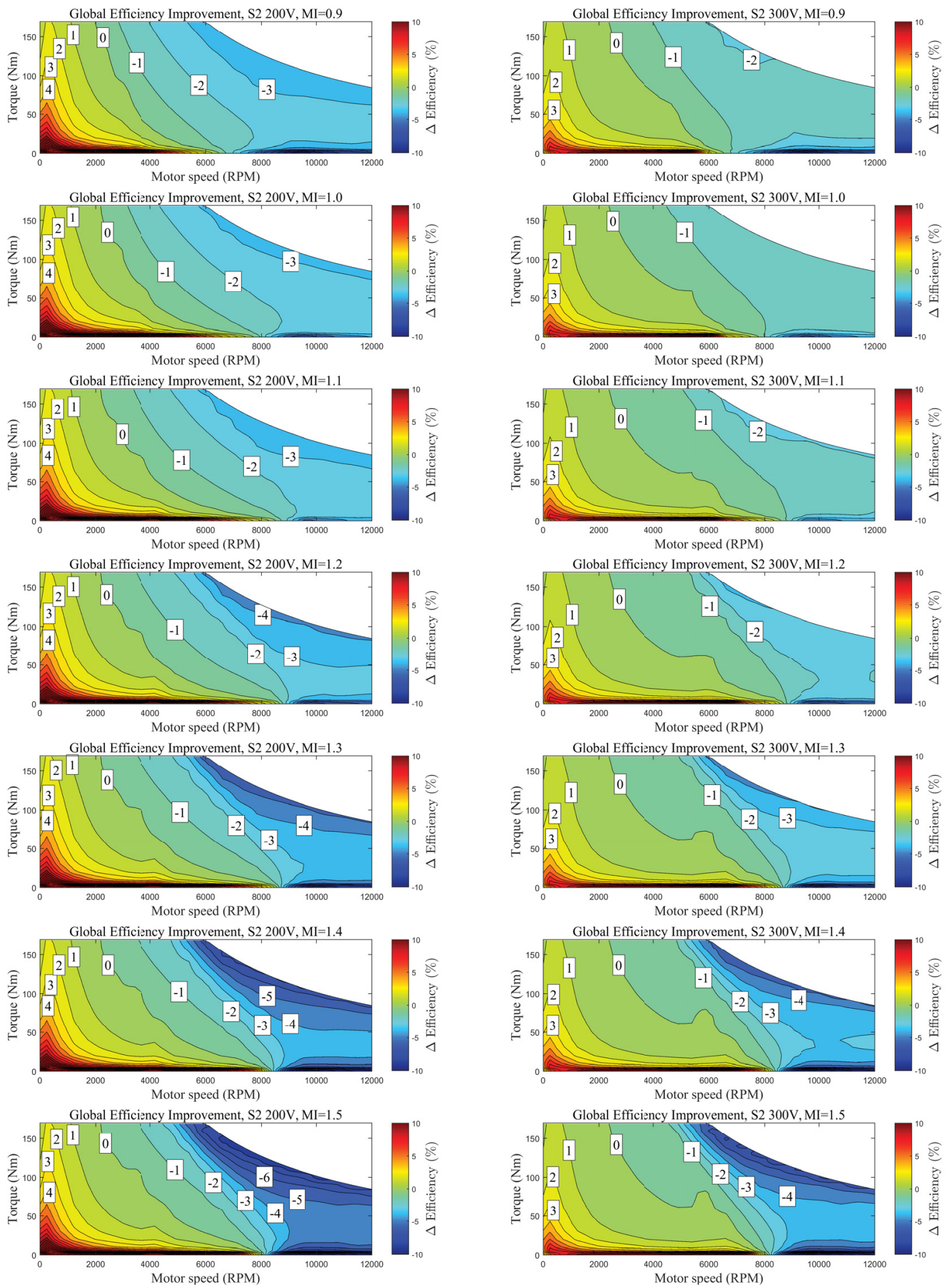

Figure 5-11: PED maps for $200 \mathrm{~V}$

Figure 5-12: PED maps for $300 \mathrm{~V}$ 

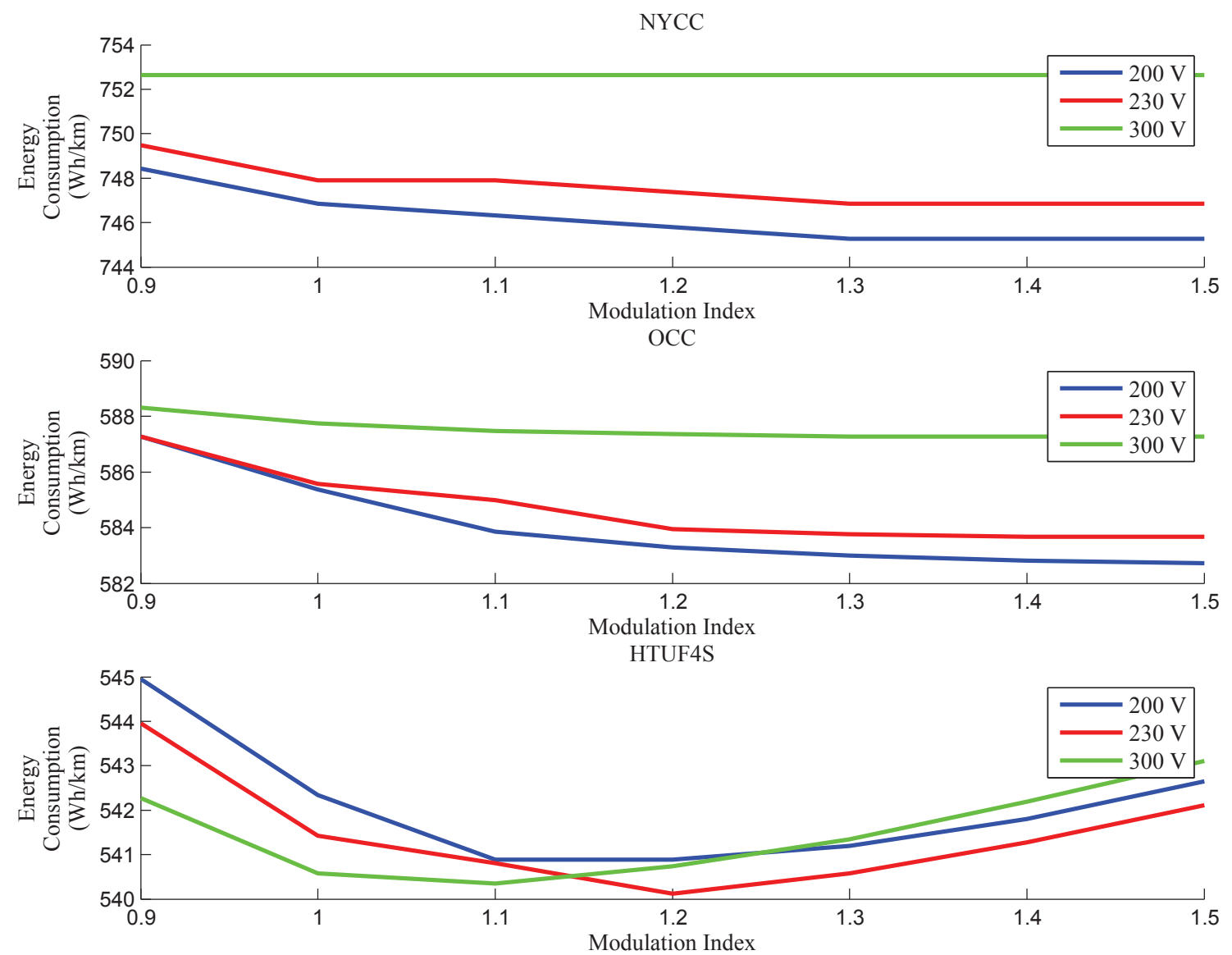

UDDS
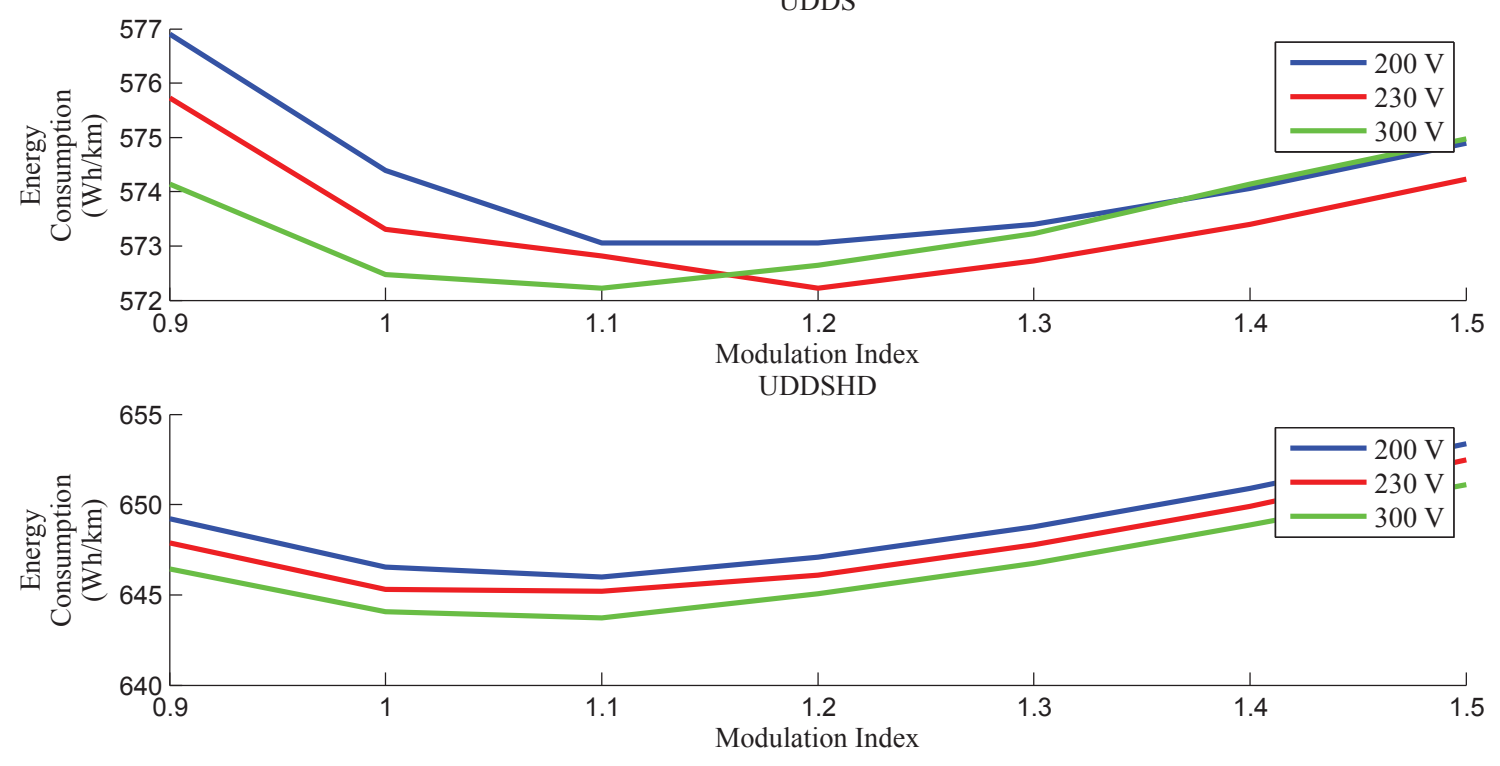

Figure 5-13: Energy consumption of S2 for various MIs and battery voltages during standard drive cycles 
Anjou_1
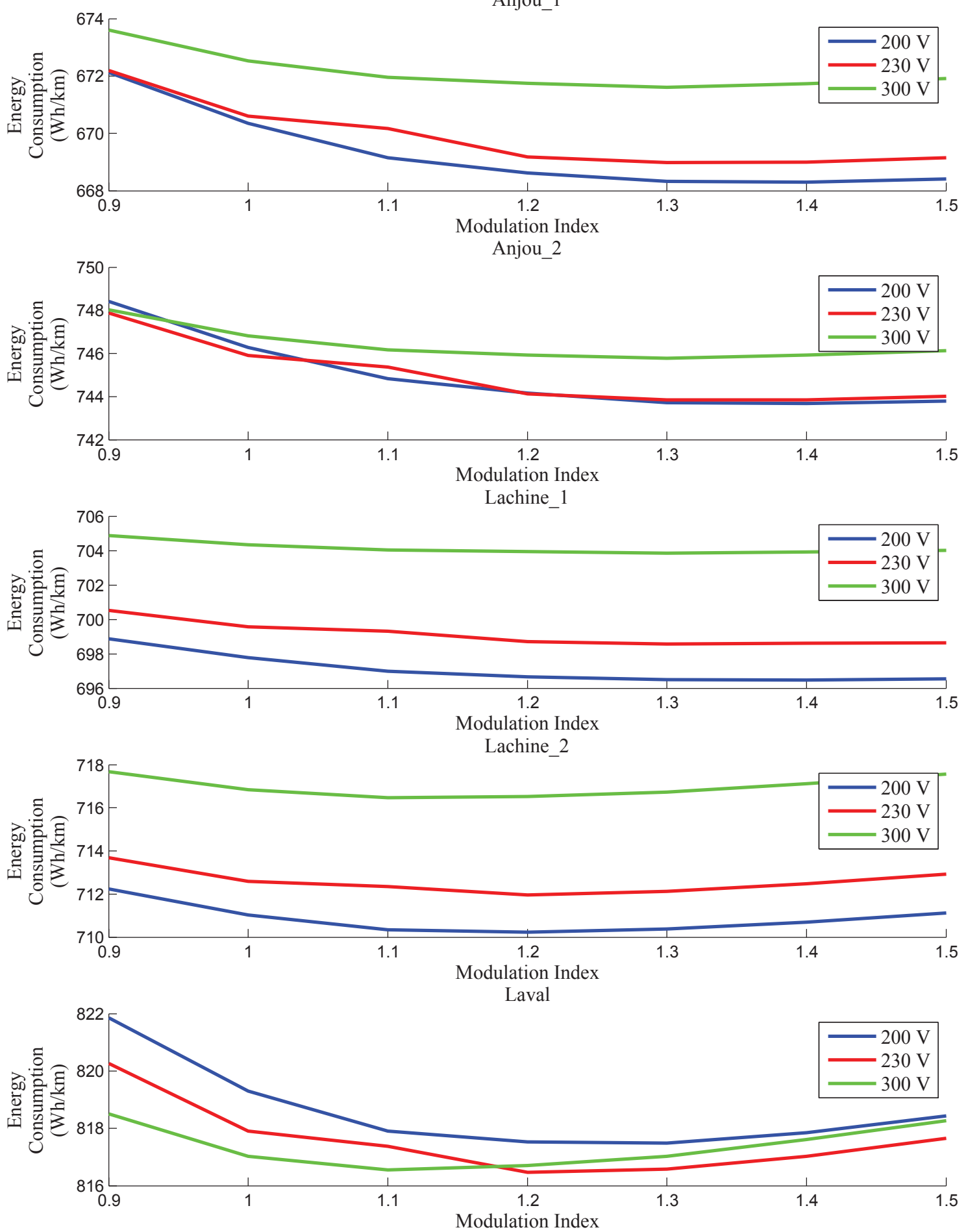

Figure 5-14: Energy consumption of S2 for various MIs and battery voltages during captured test cycles 
Table 5-4: Vehicle simulation results for Phase 4

\begin{tabular}{|c|c|c|c|c|c|c|c|}
\hline & \multicolumn{2}{|c|}{ S1 RESULTS } & \multicolumn{3}{|c|}{ S2 BEST RESULTS } & \multirow[b]{2}{*}{$\begin{array}{c}\text { Energy } \\
\text { Cons. } \\
\text { Wh/km }\end{array}$} & \multirow[b]{2}{*}{$\begin{array}{l}\text { Diff } \\
\%\end{array}$} \\
\hline & $\begin{array}{l}\text { Energy } \\
\text { Used } \\
\text { kWh }\end{array}$ & $\begin{array}{l}\text { Energy } \\
\text { Cons. } \\
\text { Wh/km }\end{array}$ & $\begin{array}{l}\text { Mod. } \\
\text { Index } \\
-\end{array}$ & $\begin{array}{l}\text { Battery } \\
\text { Voltage } \\
\text { V }\end{array}$ & $\begin{array}{l}\text { Energy } \\
\text { Used } \\
\text { kWh }\end{array}$ & & \\
\hline NYCC & 1.45 & 763 & $1.3-1.5$ & 200 & 1.42 & 745 & 2.3 \\
\hline $\mathrm{OCC}$ & 6.27 & 595 & 1.5 & 200 & 6.14 & 583 & 2.1 \\
\hline HTUF4S & 7.08 & 542 & 1.2 & 230 & 7.05 & 540 & 0.4 \\
\hline UDDS & 6.88 & 573 & 1.1 & 300 & 6.86 & 572 & 0.2 \\
\hline UDDSHD & 5.73 & 641 & 1.1 & 300 & 5.76 & 643 & -0.5 \\
\hline Anjou1 & 60.08 & 678 & 1.4 & 200 & 59.18 & 668 & 1.5 \\
\hline Anjou2 & 43.80 & 751 & 1.4 & 200 & 43.36 & 743 & 1.0 \\
\hline Lachine1 & 22.14 & 715 & 1.4 & 200 & 21.56 & 696 & 2.6 \\
\hline Lachine2 & 25.27 & 726 & 1.2 & 200 & 24.72 & 710 & 2.2 \\
\hline Laval & 86.37 & 817 & 1.2 & 230 & 86.20 & 815 & 0.2 \\
\hline
\end{tabular}

Table 5-4 summarizes the results obtained for this phase of the project. The best result among the standard drive cycles was the NYCC which was improved by $2.3 \%$ in terms of energy savings when operated at $200 \mathrm{~V}$ and an MI from 1.3 to 1.5. Among the actual routes, Lachine 1 cycle produced the best result when the system was operated at $200 \mathrm{~V}$ and an MI of 1.4. 
Table 5-5: Drive cycle metrics and results for Phases 2, 3 and 4

\begin{tabular}{|c|c|c|c|c|c|}
\hline & \multicolumn{2}{|c|}{ METRICS } & \multirow{2}{*}{$\begin{array}{c}\text { Phase } 2 \\
\text { Diff } \\
\%\end{array}$} & \multirow{2}{*}{$\begin{array}{c}\text { Phase } 3 \\
\text { Diff } \\
\%\end{array}$} & \multirow{2}{*}{$\begin{array}{c}\text { Phase } 4 \\
\text { Diff } \\
\%\end{array}$} \\
\hline & $\begin{array}{c}\text { Kinetic } \\
\text { Int. } \\
\mathrm{km}^{-1}\end{array}$ & $\begin{array}{c}\text { Avg } \\
\text { Speed } \\
\mathrm{km} / \mathrm{h}\end{array}$ & & & \\
\hline NYCC & 5.137 & 16.8 & 2.0 & 2.1 & 2.3 \\
\hline OCC & 2.231 & 24.7 & 1.6 & 1.9 & 2.1 \\
\hline HTUF4S & 0.622 & 38.8 & 0.2 & 0.4 & 0.4 \\
\hline UDDS & 0.798 & 38.9 & 0.0 & 0.2 & 0.2 \\
\hline UDDSHD & 0.378 & 45.4 & -0.8 & -0.8 & -0.5 \\
\hline Anjou1 & 2.017 & 23.8 & 1.2 & 1.4 & 1.5 \\
\hline Anjou2 & 2.119 & 26.0 & 0.7 & 1.0 & 1.0 \\
\hline Lachine1 & 1.634 & 20.0 & 2.2 & 2.3 & 2.6 \\
\hline Lachine2 & 1.352 & 19.7 & 1.9 & 1.9 & 2.2 \\
\hline Laval & 1.783 & 31.2 & 0.0 & 0.2 & 0.2 \\
\hline
\end{tabular}

Table 5-5 compares the results for all phases of the project and shows the improvement achieved in each phase of the project.

As shown, the different phases of the project have improved the energy savings but not by a large amount. The main reason behind this behavior is the fact that, even though the low RPM regions' efficiency is improved, the high RPM regions' efficiency is degraded. Although the drive cycles for a delivery truck operate mostly in the low RPM region, they still have some high RPM operation points. These points represent the high power operations and since their efficiency is lowered, they negate the gains in the low RPM region and the overall energy savings are reduced. 


\section{Chapter 6 \\ Conclusion}

In this thesis, the application of a bidirectional DC-DC converter for implementation of a variable DC voltage control in an electric delivery truck was explored. The proposed system provides higher efficiency in the low RPM region which is desirable for delivery trucks since they operate at low average speeds and go through many start and stop cycles during their daily routes.

In the first phase of the project, the $460 \mathrm{~V}$ battery pack of the original system was split in half and a battery pack with the same energy but a voltage of $230 \mathrm{~V}$ was used. A target MI of ' 1 ' was used for the control of the system. The effectiveness of the system was examined in terms of reduction of energy use throughout the OCC cycle, which most accurately represents the behavior of a delivery truck. It was demonstrated that the proposed system will result in $1.6 \%$ energy savings, which may not justify the initial cost of the new system.

In the second phase, the same two systems were examined in the context of different standard cycles and actual routes taken by a Purolator delivery truck in the greater Montreal area. It was shown that the new system is most effective for cycles that have low average speeds and high kinetic intensity. Among the standard cycles, the new system was most effective for the NYCC with an energy saving of $2.0 \%$. Among the actual route data the new system was most effective for the Lachine 1 
cycle with an energy saving of $2.2 \%$. These results still may not justify the initial cost of the system.

In the third phase of the project the same 2 systems were considered with the addition of treating the target MI as an optimization parameter. The results showed that the performance of Phase 2 can be increased by optimizing the target MI. It was demonstrated again that the new system is most effective for cycles that have low average speeds and high kinetic intensity. Among the standard cycles the new system was most effective for the NYCC with an energy saving of $2.1 \%$, which is $0.1 \%$ higher than Phase 2. Among the actual route data, the new system was most effective for the Lachine 1 with the energy saving of $2.3 \%$ which is $0.1 \%$ higher than the results obtained based on Phase 2. These results still may not justify the initial cost of the system.

In the fourth phase of the project, three battery packs with almost the same amount of energy and different operating voltages were designed. The main idea in this section was to treat both the battery voltage and the target MI as optimization parameters. In this phase the performance obtained in Phase 3 can be further improved. Among the standard cycles, the new system was most effective for the NYCC with the energy saving of $2.3 \%$. Among the actual route data, the new system was most effective for the Lachine 1 cycle with the energy saving of $2.6 \%$. These results still may not justify the initial cost of the system.

Overall, the proposed system introduced an improvement for an electric delivery truck. However, even with several advanced levels of optimization the system did not 
manage to introduce enough energy savings in order to make it a suitable potential candidate for the powertrain of an electric delivery truck.

The optimization for target MI was run in intervals of 0.1 from an MI of 0.9 to an MI of 1.5. The optimization of the battery voltage was run for three values of 200 $\mathrm{V}, 230 \mathrm{~V}$ and $300 \mathrm{~V}$. Therefore, in order to fully examine the system, future work should consist of examining the system for more MIs and battery voltages with an increase in resolution.

Future investigation should include the implementation of a dynamic control strategy that manipulates the target MI throughout a whole cycle and operates the system for any point in the torque speed envelope at its optimal MI.

The application of a 2-speed transmission could also be studied, since using this technology it is possible to operate the system in its most efficient region (i.e. low RPM) for a wider range of vehicle speeds.

Finally, other DC-DC converter topologies with different battery pack structures should be studied, since they may offer superior results in the low RPM range compared to the boost converter that was studied in this thesis. For example, buck converters could be of particular interest since in this application the boost converters were not used for the expansion of the torque-speed envelope or battery voltage drop compensation. Because a buck converter can lower the DC bus voltage to a value below the battery voltage, it is possible to operate the inverter at the desired MI at any speed, including the low RPM region which was not possible with a boost converter. Another DC-DC converter topology which should be studied in the future work is the buck-boost. This topology can provide the benefits of a boost converter 
and a buck converter at the same time and therefore the optimal MI can be achieved at all RPMs and when battery voltage drops it can be boosted to the nominal value. 


\section{References}

[1] M. Ehsani, Y. Gao, and A. Emadi, Modern electric, hybrid electric, and fuel cell vehicles: fundamentals, theory, and design. CRC press, 2009.

[2] A. Najmabadi, K. Humphries, and B. Boulet, "Application of a bidirectional DC-DC in an electric powertrain for medium duty delivery trucks," in International Electric Machines and Drives Conference (IEMDC), 2015 IEEE, pp. 866871, IEEE.

[3] A. Najmabadi, K. Humphries, and B. Boulet, "Implementation of a bidirectional DC-DC in electric powertrains for drive cycles used by medium duty delivery trucks," in Energy Conversion Congress and Exposition (ECCE), 2015 IEEE, pp. 1338-1345, IEEE.

[4] A. Najmabadi, K. Humphries, and B. Boulet, "Effects of target modulation index on variable DC voltage control for electric powertrains in medium duty delivery trucks," in Vehicle Power and Propulsion Conference (VPPC), 2015 IEEE, IEEE.

[5] R. H. Park, "Two-reaction theory of synchronous machines generalized method of analysis-part i," American Institute of Electrical Engineers, Transactions of the, vol. 48, no. 3, pp. 716-727, 1929.

[6] R. H. Park, "Two-reaction theory of synchronous machines-ii," American Institute of Electrical Engineers, Transactions of the, vol. 52, no. 2, pp. 352-354, 1933.

[7] R. E. Vowels, "Transient analysis of synchronous machines," Proceedings of the IEE - Part IV: Institution Monographs, vol. 99, no. 3, pp. 204-216, 1952.

[8] R. DOLEEK, J. Novak, and O. ERN, "Traction permanent magnet synchronous motor torque control with flux weakening," Radioengineering, vol. 18, no. 4, pp. 601-605, 2009. 
[9] N. Mohan, T. M. Undeland, and W. P. Robbins, Power Electronics: Converters, Applications, and Design. John Wiley \& Sons, Inc., 3rd ed., 2003.

[10] B. Boulet, Fundamentals of signals and systems. Charles River Media, 2005.

[11] R. Barnitt, "Fedex express gasoline hybrid electric delivery truck evaluation: 12-month report," 2011.

[12] M. P. O’Keefe, A. Simpson, K. J. Kelly, and D. S. Pedersen, "Duty cycle characterization and evaluation towards heavy hybrid vehicle applications," report, SAE Technical Paper, 2007.

[13] J. Hsu, C. Ayers, and C. Coomer, "Report on toyota/prius motor design and manufacturing assessment," report, Oak Ridge National Laboratory (ORNL), 2004.

[14] C. Ayers, "Evaluation of 2004 toyota prius hybrid electric drive system interim report," report, Oak Ridge National Laboratory (ORNL), 2004.

[15] R. H. Staunton, C. W. Ayers, L. D. Marlino, J. N. Chiasson, and T. A. Burress, "Evaluation of 2004 toyota prius hybrid electric drive system," report, Oak Ridge National Laboratory (ORNL), 2006.

[16] T. A. Burress, S. L. Campbell, C. Coomer, C. W. Ayers, A. A. Wereszczak, J. P. Cunningham, L. D. Marlino, L. E. Seiber, and H.-T. Lin, "Evaluation of the 2010 toyota prius hybrid synergy drive system," report, Oak Ridge National Laboratory (ORNL); Power Electronics and Electric Machinery Research Facility, 2011.

[17] S. S. Williamson, S. M. Lukic, and A. Emadi, "Comprehensive drive train efficiency analysis of hybrid electric and fuel cell vehicles based on motorcontroller efficiency modeling," Power Electronics, IEEE Transactions on, vol. 21, no. 3, pp. 730-740, 2006.

[18] M. B. Camara, H. Gualous, F. Gustin, A. Berthon, and B. Dakyo, "Dc/dc converter design for supercapacitor and battery power management in hybrid vehicle applications - polynomial control strategy," Industrial Electronics, IEEE Transactions on, vol. 57, no. 2, pp. 587-597, 2010.

[19] A. Khaligh and Z. Li, "Battery, ultracapacitor, fuel cell, and hybrid energy storage systems for electric, hybrid electric, fuel cell, and plug-in hybrid electric 
vehicles: State of the art," Vehicular Technology, IEEE Transactions on, vol. 59, no. 6, pp. 2806-2814, 2010.

[20] J. Silvestre, "Half-bridge bidirectional dc-dc converter for small electric vehicle," in Power Electronics, Electrical Drives, Automation and Motion, 2008. SPEEDAM 2008. International Symposium on, pp. 884-888, IEEE.

[21] K. P. Yalamanchili and M. Ferdowsi, "Review of multiple input dc-dc converters for electric and hybrid vehicles," in Vehicle Power and Propulsion, 2005 IEEE Conference, pp. 160-163, IEEE.

[22] A. Di Napoli, F. Crescimbini, S. Rodo, and L. Solero, "Multiple input dc-dc power converter for fuel-cell powered hybrid vehicles," in Power Electronics Specialists Conference, 2002. pesc 02. 2002 IEEE 33rd Annual, vol. 4, pp. 16851690, IEEE.

[23] A. Di Napoli, F. Crescimbini, L. Solero, F. Caricchi, and F. G. Capponi, "Multiple-input dc-dc power converter for power-flow management in hybrid vehicles," in Industry Applications Conference, 2002. 37th IAS Annual Meeting. Conference Record of the, vol. 3, pp. 1578-1585, IEEE.

[24] S. M. Lukic, S. G. Wirasingha, F. Rodriguez, J. Cao, and A. Emadi, "Power management of an ultracapacitor/battery hybrid energy storage system in an hev," in Vehicle Power and Propulsion Conference, 2006. VPPC'06. IEEE, pp. 1-6, IEEE.

[25] S. M. Lukic, J. Cao, R. C. Bansal, F. Rodrguez, and A. Emadi, "Energy storage systems for automotive applications," Industrial electronics, IEEE Transactions on, vol. 55, no. 6, pp. 2258-2267, 2008.

[26] J. O. Estima and A. J. Marques Cardoso, "Efficiency analysis of drive train topologies applied to electric/hybrid vehicles," Vehicular Technology, IEEE Transactions on, vol. 61, no. 3, pp. 1021-1031, 2012.

[27] J. O. Estima and A. M. Cardoso, "Performance analysis of a pmsm drive for hybrid electric vehicles," in Electrical Machines (ICEM), 2010 XIX International Conference on, pp. 1-6, IEEE.

[28] J. O. Estima and A. Cardoso, "Thermal evaluation of different drive train topologies for electric/hybrid vehicles," in Electrical Machines (ICEM), 2014 International Conference on, pp. 2454-2460, IEEE. 
[29] T. Schoenen, M. S. Kunter, M. D. Hennen, and R. W. De Doncker, "Advantages of a variable dc-link voltage by using a dc-dc converter in hybrid-electric vehicles," in Vehicle Power and Propulsion Conference (VPPC), 2010 IEEE, pp. 1-5, IEEE.

[30] S. Tenner, S. Gunther, and W. Hofmann, "Loss minimization of electric drive systems using a dc/dc converter and an optimized battery voltage in automotive applications," in Vehicle Power and Propulsion Conference (VPPC), 2011 IEEE, pp. 1-7, IEEE.

[31] H. v. Hoek, M. Boesing, D. v. Treek, T. Schoenen, and R. W. D. Doncker, "Power electronic architectures for electric vehicles," in VDE-Kongress 2010 , VDE VERLAG GmbH.

[32] F. Caricchi, F. Crescimbini, G. Noia, and D. Pirolo, "Experimental study of a bidirectional dc-dc converter for the dc link voltage control and the regenerative braking in pm motor drives devoted to electrical vehicles," in Applied Power Electronics Conference and Exposition, 1994. APEC'94. Conference Proceedings 1994., Ninth Annual, pp. 381-386, IEEE.

[33] R. A. Barnitt, A. D. Brooker, and L. Ramroth, "Model-based analysis of electric drive options for medium-duty parcel delivery vehicles," 2010.

[34] ADVISOR, "Advisor: Advanced vehicle simulator documentation," 2003.

[35] United States Postal Service, "Electrification of delivery vehicles," report, U.S. Postal Service Office of Inspector General, 2009.

[36] R. M. Schupbach and J. C. Balda, "Comparing dc-dc converters for power management in hybrid electric vehicles," in Electric Machines and Drives Conference, 2003. IEMDC'03. IEEE International, vol. 3, pp. 1369-1374, IEEE.

[37] R. Schupbach and J. Balda, "35 kw ultracapacitor unit for power management of hybrid electric vehicles: bidirectional dc-dc converter design," in Power Electronics Specialists Conference, 2004. PESC 04. 2004 IEEE 35th Annual, vol. 3, pp. 2157-2163, IEEE. 